\title{
Symmetry-enforced band crossings in tetragonal materials: Dirac and Weyl degeneracies on points, lines, and planes
}

\author{
Moritz M. Hirschmann $\odot,{ }^{1}$ Andreas Leonhardt $\odot,{ }^{1}$ Berkay Kilic $\odot,{ }^{1,2}$ Douglas H. Fabini $\odot,{ }^{1}$ and Andreas P. Schnyder ${ }^{1, *}$ \\ ${ }^{1}$ Max-Planck-Institut für Festkörperforschung, Heisenbergstrasse 1, D-70569 Stuttgart, Germany \\ ${ }^{2}$ Institute for Functional Materials and Quantum Technologies, University of Stuttgart, Pfaffenwaldring 57, D-70550 Stuttgart, Germany
}

(Received 15 February 2021; accepted 4 May 2021; published 28 May 2021)

\begin{abstract}
We study the occurrence of symmetry-enforced topological band crossings in tetragonal crystals with strong spin-orbit coupling and time-reversal symmetry. By computing the momentum dependence of the symmetry eigenvalues and the global band topology in the entire Brillouin zone, we determine all symmetry-enforced band crossings in tetragonal space groups. In particular, we classify all Dirac and Weyl degeneracies on points, lines, and planes, and find a rich variety of topological degeneracies. This includes, among others, double Weyl points, fourfold double Weyl points, fourfold quadruple Weyl points, Weyl and Dirac nodal lines, as well as topological nodal planes. For the space groups with symmetry-enforced Weyl points, we determine the minimal number of Weyl points for a given band pair and, remarkably, find that materials in space groups 119 and 120 can have band pairs with only two Weyl points in the entire Brillouin zone. This simplifies the contribution of the Weyl points to the topological responses, which would be useful for device applications. Using the classification of symmetry-enforced band crossings, we perform an extensive database search for candidate materials with tetragonal space groups. Notably, we find that $\mathrm{Ba}_{5} \mathrm{In}_{4} \mathrm{Bi}_{5}$ and $\mathrm{NaSn}_{5}$ exhibit twofold and fourfold Weyl nodal lines, respectively, which cross the Fermi energy. $\mathrm{Hf}_{3} \mathrm{Sb}$ and $\mathrm{Cs}_{2} \mathrm{Tl}_{3}$ have band pairs with few number of Weyl points near the Fermi energy. Furthermore, we show that $\mathrm{Ba}_{3} \mathrm{Sn}_{2}$ has Weyl points with an accordion dispersion and topological nodal planes, while $\mathrm{AuBr}$ and $\mathrm{Tl}_{4} \mathrm{PbSe}_{3}$ possess Dirac points with hourglass dispersions. For each of these candidate materials we present the $a b$ initio band structures and discuss possible experimental signatures of the nontrivial band topology.
\end{abstract}

DOI: 10.1103/PhysRevMaterials.5.054202

\section{INTRODUCTION}

Topological semimetals exhibit protected band crossings near the Fermi energy, which carry nonzero topological charges [1-8]. The existence of such band crossings has been recognized early on in the development of solid-state physics [9], but their importance was appreciated only recently. Indeed, over the last few years it was shown that topological band crossings lead to a number of interesting phenomena, such as, unusual magnetotransport [10], intrinsic anomalous Hall effects [11], large thermopower [12], exotic surface states [13-19], and various responses related to quantum anomalies [20-23]. Due to these unusual properties, topological semimetals hold great potential for novel device applications [24]. For example, the helical nature of the electronic states can be utilized for low-dissipation transport [7]. Using the spin-momentum locking of the surface states, low-consumption spintronic devices $[25,26]$ and magnetic memory devices [27] can be constructed. It may also be possible to build topological field effect transistors, by controlling

\footnotetext{
*a.schnyder@fkf.mpg.de
}

Published by the American Physical Society under the terms of the Creative Commons Attribution 4.0 International license. Further distribution of this work must maintain attribution to the author(s) and the published article's title, journal citation, and DOI. Open access publication funded by the Max Planck Society. the phase transitions of topological semimetals [28]. The high photosensitivity of topological semimetals may provide a possibility for building ultrafast broadband photodetectors [29-31]. Besides, many topological semimetals exhibit large thermoelectric responses, which makes them promising for high-efficiency energy converters and thermal detectors [32].

Despite these extensive research activities, there is still a lack of suitable materials for device applications. In this paper, we use the principle of symmetry-enforced band crossings to look for new topological materials. That is, we investigate under which circumstances nonsymmorphic symmetries lead to enforced band crossings on high-symmetry points, lines, or planes [10,33-45]. We focus on space groups (SGs) in the tetragonal crystal system, which exhibit fourfold (screw) rotations about the $z$ axis. Previously, we have applied this strategy to classify symmetry-enforced band crossings in materials with hexagonal and trigonal SG symmetries [43,44]. A future work will be concerned with orthorhombic SGs [45].

We summarize our results in Tables I and II, which classify all possible symmetry-enforced band crossings in tetragonal materials with strong spin-orbit coupling. We find a large variety of different types of topological band degeneracies on points, lines, and planes. These include Dirac and Weyl points, Dirac nodal lines, twofold and fourfold Weyl nodal lines, as well as topological nodal planes. Regarding the symmetryenforced Weyl points, we find four different variants, namely, single Weyl points with a linear band crossing and Chern number $|\mathcal{C}|=1$, double Weyl points with a quadratic band crossing along two directions and $|\mathcal{C}|=2$, fourfold double 
Weyl points with a fourfold degeneracy and $|\mathcal{C}|=2$, and fourfold quadruple Weyl points with a fourfold degeneracy and $|\mathcal{C}|=4$ (Sec. IV). The degeneracies along nodal lines also come in different varieties: Dirac nodal lines, fourfold Weyl nodal lines, and twofold Weyl nodal lines, which can form nodal chains or "armillary spheres" (Sec. VI). Additionally, there are "almost movable nodal lines," which are pinned to high-symmetry points but otherwise freely movable (Sec. VI A). ${ }^{1}$ Finally, for the nodal planes, we find that they occur on the Brillouin zone (BZ) boundaries and are

\footnotetext{
${ }^{1}$ We note that the existence of these almost movable nodal lines cannot be derived solely form the compatibility relations of irreps.
}

TABLE I. Classification of band crossings in noncentrosymmetric tetragonal crystals with strong spin-orbit coupling. The first column lists the tetragonal space groups (SG) that lack inversion symmetry by their number and symbol. The second and third columns list the positions of Weyl points (see Sec. IV A) and Weyl nodal lines (see Sec. VI), which are movable on the indicated rotation axis or mirror plane. For Weyl lines we group the high-symmetry points, e.g., "X, R," or lines, e.g. " $\Gamma-Z-R$," into sets with the same mirror eigenvalue pairing. The sets of points for different eigenvalues are separated by semicolons. Note fourfold degeneracies fall into neither category, which is indicated by italicized type, e.g., $Z$ in $\Gamma-Z$-R. On a path between any two points with different eigenvalues nodal lines are found. If a point (instead of a line) is given in the first eigenvalue set an almost nodal line passes through it (see Sec. VI A). For example, $(\mathrm{P}, \Gamma-\mathrm{Z} ;-)(2)$ denotes a nodal line passing through $\mathrm{P}$ situated in the mirror plane containing $\mathrm{P}$ and $\Gamma-\mathrm{Z}$. The number in brackets corresponds to the number of bands connected by the given feature. The fourth column denotes points in reciprocal space with a fourfold degeneracy. The fifth column gives the lowest possible number of point crossings which carry a nonzero chirality, thereby including fourfold degeneracies but excluding nodal planes. Several numbers are given if the minimal number of Weyl points depends on the band index. The second to last column lists conditions on $\mathbf{k}$ for which all bands are twofold degenerate (see the discussion of nodal planes in Sec. VIII). The last column contains a selection of noteworthy features of the respective SG. Nodal points allowing for irreducible representations of different dimensions are marked by $(*)$.

\begin{tabular}{|c|c|c|c|c|c|c|c|}
\hline & SG & Movable Weyl points & Movable Weyl lines & Fourfold points & No. Weyl & Nodal planes & Notable features \\
\hline 75 & $P 4$ & & & & 8 & & \\
\hline 76 & $P 4_{1}$ & $\Gamma-\mathrm{Z}(8), \mathrm{M}-\mathrm{A}(8), \mathrm{X}-\mathrm{R}(4)$ & & & 4,8 & $k_{z}=\pi$ & Double Weyl \\
\hline 77 & $P 4_{2}$ & $\Gamma-\mathrm{Z}(4), \mathrm{M}-\mathrm{A}(4)$ & & & 4,8 & & Double Weyl \\
\hline 78 & $P 4_{3}$ & $\Gamma-\mathrm{Z}(8), \mathrm{M}-\mathrm{A}(8), \mathrm{X}-\mathrm{R}(4)$ & & & 4,8 & $k_{z}=\pi$ & Double Weyl \\
\hline 79 & $I 4$ & & & & 10 & & Weyl at P \\
\hline 80 & $I 4_{1}$ & $\Gamma-\mathrm{Z}-\mathrm{M}(4)$ & & & 4,8 & & Double Weyl, Weyl at $\mathrm{P}\left({ }^{*}\right)$ \\
\hline 81 & $P \overline{4}$ & & & & 4 & & \\
\hline 82 & $I \overline{4}$ & & & & 6 & & \\
\hline 89 & $P 422$ & & & & 8 & & \\
\hline 90 & $P 42_{1} 2$ & $\Gamma-\mathrm{X}(4), \mathrm{Z}-\mathrm{R}(4)$ & & $\mathrm{A}, \mathrm{M}$ & 2,10 & $k_{x}, k_{y}=\pi$ & Fourfold Weyl \\
\hline 91 & $P 4_{1} 22$ & $\Gamma-\mathrm{Z}(8), \mathrm{M}-\mathrm{A}(8), \mathrm{X}-\mathrm{R}(4)$ & & & 4,8 & $k_{z}=\pi$ & Double Weyl \\
\hline 92 & $P 4_{1} 2_{1} 2$ & $\Gamma-\mathrm{Z}(8), \Gamma-\mathrm{X}(4)$ & & $\mathrm{M}, \mathrm{R}, \mathrm{A}\left({ }^{*}\right)$ & $1,3,9$ & $k_{x}, k_{y}, k_{z}=\pi$ & $\begin{array}{c}\text { Top. nodal plane, } \\
\text { twofold/fourfold } \\
\text { (double/quadruple) Weyl }\end{array}$ \\
\hline 93 & $P 4_{2} 22$ & $\Gamma-\mathrm{Z}(4), \mathrm{M}-\mathrm{A}(4)$ & & & 4,8 & & double Weyl \\
\hline 94 & $P 4_{2} 2_{1} 2$ & $\Gamma-\mathrm{Z}(4), \Gamma-\mathrm{X}(4), \mathrm{Z}-\mathrm{R}(4)$ & & $\mathrm{A}, \mathrm{M}$ & 2,12 & $k_{x}, k_{y}=\pi$ & $\begin{array}{l}\text { Top. nodal plane, } \\
\text { double/fourfold Weyl }\end{array}$ \\
\hline 95 & $P 4_{3} 22$ & $\Gamma-\mathrm{Z}(8), \mathrm{M}-\mathrm{A}(8), \mathrm{X}-\mathrm{R}(4)$ & & & 4,8 & $k_{z}=\pi$ & Double Weyl \\
\hline 96 & $P 4_{3} 2_{1} 2$ & $\Gamma-\mathrm{Z}(8), \Gamma-\mathrm{X}(4)$ & & $\mathrm{M}, \mathrm{R}, \mathrm{A}(*)$ & $1,3,9$ & $k_{x}, k_{y}, k_{z}=\pi$ & See SG 92 \\
\hline 97 & $I 422$ & & & & 10 & & Weyl at $P$ \\
\hline 98 & $I 4_{1} 22$ & $\Gamma-\mathrm{Z}-\mathrm{M}(4)$ & & & 4,8 & & Weyl at $\mathrm{P}(*)$ \\
\hline 99 & $P 4 m m$ & & & & & & \\
\hline 100 & $P 4 b m$ & & $(\Gamma-Z ; X, R)(4)$ & $\mathrm{M}, \mathrm{A}$ & & & \\
\hline 101 & $P 4_{2} \mathrm{~cm}$ & & & $\mathrm{Z}, \mathrm{A}, \mathrm{R}$ & & & \\
\hline 102 & $P 4_{2} \mathrm{~nm}$ & & $(\Gamma-Z-\mathrm{R} ; \mathrm{X})(4),(\mathrm{X}-M-\mathrm{A} ; \mathrm{R})(4)$ & $\mathrm{Z}, \mathrm{M}$ & & & Nodal chain metal \\
\hline 103 & $P 4 c c$ & & & $\mathrm{Z}, \mathrm{R}, \mathrm{A}$ & & & \\
\hline 104 & $P 4 n c$ & & $(\Gamma-Z-\mathrm{R} ; \mathrm{X})(4),(\mathrm{X}-M-A ; \mathrm{R})(4)$ & $\mathrm{Z}, \mathrm{M}, \mathrm{A}$ & & & Nodal chain metal \\
\hline 105 & $P 4_{2} m c$ & & & $\mathrm{Z}, \mathrm{A}$ & & & \\
\hline 106 & $P 4_{2} b c$ & & $(\Gamma-Z ; X, R)(4)$ & $\mathrm{Z}, \mathrm{A}, \mathrm{M}, \mathrm{M}-\mathrm{A}(8)$ & & & Movable fourfold point \\
\hline 107 & $I 4 m m$ & & $\left(\mathrm{~N}, \Gamma-\mathrm{Z}, \mathrm{M}-\mathrm{Z}_{1} ;-\right)(2)$ & & & & \\
\hline 108 & $I 4 \mathrm{~cm}$ & & $\left(\Gamma-\mathrm{Z}, \mathrm{M}-\mathrm{Z}_{1} ; \mathrm{N}\right)(4)$ & $\mathrm{P}$ & & & Fourfold at $\mathrm{P}$ \\
\hline 109 & $I 4_{1} m d$ & & $(\Gamma-\mathrm{Z} ; \mathrm{X})(4),\left(\mathrm{N}, \Gamma-\mathrm{Z}, M-\mathrm{Z}_{1} ;-\right)(2)$ & M & & & $\mathrm{P}(*)$, nodal chain metal \\
\hline 110 & $I 4_{1} c d$ & & $\begin{array}{c}(\Gamma-\mathrm{Z}, \mathrm{P} ; \mathrm{X})(4),(\Gamma-\mathrm{Z}, \mathrm{X} ; \mathrm{P})(4) \\
(\mathrm{X}-\mathrm{M} ; \mathrm{P})(4),\left(\Gamma-\mathrm{Z}, M-\mathrm{Z}_{1} ; \mathrm{N}\right)(4) \\
(\mathrm{P}, \Gamma-\mathrm{Z} ;-)(2),\left(\mathrm{P}, \mathrm{X}-\mathrm{M}-\mathrm{Z}_{1} ;-\right)(2)\end{array}$ & M & & & $\begin{array}{c}8 \mathbb{N} \text { bands, } \\
\text { in-gap nodal lines }\end{array}$ \\
\hline
\end{tabular}


TABLE I. (Continued.)

\begin{tabular}{|c|c|c|c|c|c|c|c|}
\hline \multicolumn{2}{|c|}{ SG } & \multirow[t]{2}{*}{ Movable Weyl points } & \multirow[t]{2}{*}{ Movable Weyl lines } & \multirow[t]{2}{*}{ Fourfold points } & \multirow{2}{*}{$\frac{\text { No. Weyl }}{4}$} & \multirow[t]{2}{*}{ Nodal planes } & \multirow[t]{2}{*}{ Notable features } \\
\hline 111 & $P \overline{4} 2 m$ & & & & & & \\
\hline 112 & $P \overline{4} 2 c$ & & & $\mathrm{Z}, \mathrm{A}$ & 4 & & \\
\hline 113 & $P \overline{4} 2_{1} m$ & $\Gamma-\mathrm{X}(4), \mathrm{Z}-\mathrm{R}(4)$ & & $\overline{\mathrm{MA}}$ & 8 & $k_{x}, k_{y}=\pi$ & Fourfold line \\
\hline 114 & $P \overline{4} 2_{1} c$ & $\Gamma-\mathrm{X}(4)$ & & $\mathrm{Z}, \overline{\mathrm{MA}}$ & 4 & $k_{x}, k_{y}=\pi$ & Fourfold line \\
\hline 115 & $P \overline{4} m 2$ & & & & & & \\
\hline 116 & $P \overline{4} c 2$ & & & $\mathrm{Z}, \mathrm{A}, \mathrm{R}$ & & & \\
\hline 117 & $P \overline{4} b 2$ & & $(\Gamma-Z ; X, R)(4)$ & $\mathrm{A}, \mathrm{M}$ & & & \\
\hline 118 & $P \overline{4} n 2$ & A-R(4), R-X(4) & $\begin{array}{l}(\Gamma-Z-\mathrm{R} ; \mathrm{X})(4) \\
(\mathrm{X}-M-\mathrm{A} ; \mathrm{R})(4)\end{array}$ & $\mathrm{Z}, \mathrm{M}$ & & & Nodal chain metal \\
\hline 119 & $I \overline{4} m 2$ & & $\left(\mathrm{~N}, \Gamma-\mathrm{Z}, \mathrm{M}-\mathrm{Z}_{1} ;-\right)(2)$ & & 2 & & \\
\hline 120 & $I \overline{4} c 2$ & $\mathrm{X}-\mathrm{P}(4)$ & $\left(\Gamma-\mathrm{Z}, \mathrm{M}-\mathrm{Z}_{1} ; \mathrm{N}\right)(4)$ & & 2 & & \\
\hline 121 & $I \overline{4} 2 m$ & & & & 4 & & \\
\hline 122 & $I \overline{4} 2 d$ & & $(\Gamma-Z ; X)(4)$ & M & 4 & & Nodal chain metal \\
\hline
\end{tabular}

enforced by the combination of screw rotation with timereversal symmetry. The nontrivial topology of these nodal planes follows from the Nielsen-Ninomiya fermion doubling theorem $[46,47]$ together with the global band topology (i.e., the topological charge of all band crossings of a given band pair) (see Sec. VIII).

With these classification results in hand, we proceed to identify materials that exhibit the aforementioned symmetryenforced band crossings. For that purpose, we perform extensive database searches for materials with strong spinorbit coupling and the relevant space-group symmetries (Sec. II B and Fig. 2). Our search yields seven candidate materials, whose band structures are studied in detail using density functional theory (DFT) calculations. In particular, we find that $\mathrm{Ba}_{5} \mathrm{In}_{4} \mathrm{Bi}_{5}$ possesses Weyl nodal lines close to the Fermi energy, which form nodal chains (Sec. VIB). $\mathrm{NaSn}_{5}$ exhibits fourfold nodal lines crossing the Fermi energy (Sec. VII). $\mathrm{Ba}_{3} \mathrm{Sn}_{2}$ has single and double Weyl points with accordion dispersions and topological nodal planes (Secs. IV C and VIII A), while $\mathrm{Hf}_{3} \mathrm{Sb}$ and $\mathrm{Cs}_{2} \mathrm{Tl}_{3}$ exhibit a small number of Weyl points close to the Fermi energy (Secs. III A 1 and III A 2). The centrosymmetric compounds $\mathrm{Tl}_{4} \mathrm{PbSe}_{3}$ and $\mathrm{AuBr}$ possess movable Dirac points forming hourglass dispersions (Secs. V B and V C).

The remainder of this paper is organized as follows. In Sec. II we introduce our naming conventions for the different topological band degeneracies and explain our notation for the symmetry operators and high-symmetry points (Sec. II A). Moreover, we explain the details of our database search for candidate materials (Sec. II B). We find candidate materials for seven of the tetragonal SGs. Their band structures and topological features are presented in those sections, where the corresponding SGs are analyzed. In Sec. III we study band degeneracies that are enforced by Kramers theorem. We show that Kramers theorem leads to Weyl points at time-reversal invariant momenta (TRIMs) (Sec. III A), as well as to point and line degeneracies on other high-symmetry points and lines, respectively (Sec. III B). Section IV is concerned with hourglass and accordion dispersions (Sec. IV A), as well as Weyl points that are symmetry enforced by screw rotations. Depending on the tetragonal SG, these Weyl points are of the following types: single Weyl points (Sec. IV B), double Weyl points (Sec. IV C), fourfold double Weyl points (Sec. IV D), and fourfold quadruple Weyl points (Sec. IV E). In Sec. V we discuss movable fourfold points and Dirac points, whose existence is enforced by screw rotations combined with glide reflections or other multiple (non)symmorphic symmetries. Sections VI and VII are devoted to the study of twofold and fourfold Weyl nodal lines, respectively. In particular, we investigate nodal lines forming chains of connected rings (Sec. VIB) and armillary spheres (Sec. VIC). In Sec. VIII we study nodal planes, i.e., band degeneracies on twodimensional planes at the BZ boundary, which are enforced by screw rotations together with time-reversal symmetry. The nontrivial topological charge of these nodal planes is inferred from the global band topology of all band crossings in the BZ. Finally, in Sec. IX we analyze band crossings protected by off-centered symmetries, i.e., by the combination of screw rotation (or glide mirror) with inversion. The conclusions and outlook of our work are given in Sec. X. Additional band structure calculations are presented in Appendix A. In Appendix B we discuss tight-binding models and their topological surface states for some tetragonal SGs. In Appendix C we derive effective Hamiltonians describing the low-energy physics near different types of topological band crossings.

\section{PRELIMINARIES}

Before presenting the results of our classification, we start in this section by introducing our conventions for the naming of topological band degeneracies and by explaining the notations for the symmetry operators (Sec. II A). We also give a brief discussion on how the database search for candidate materials was performed (Sec. II B).

There are in total 68 tetragonal space groups, of which 49 have primitive lattices ( $\mathrm{P}$ tetragonal), and the remaining 19 have body-centered lattices (I tetragonal). All tetragonal space groups possess a fourfold rotation symmetry around the $z$ axis. The BZs for the tetragonal space groups are shown in Fig. 1. Tetragonal lattices contain two independent lattice constants $a$ and $c$, which correspond to the breadth and height of the conventional unit cell along the $x$ and $z$ directions, 
TABLE II. Classification of enforced band crossings in centrosymmetric tetragonal crystals with strong spin-orbit coupling. The first column lists the tetragonal space groups including inversion symmetry by their number and symbol. The second and third columns list the positions of Dirac points and Dirac lines. Here, R-Z(4) refers to a movable Dirac point on the line connecting R and Z in an hourglass structure comprising four spin-degenerate bands (see Secs. VB and V C). The fourth and fifth columns list lines and planes on which accidental points and lines are possible, respectively. Here, the fourfold rotation axis $\Gamma-\mathrm{Z}$ and $\mathrm{M}-\mathrm{A}$ are omitted because they always allow accidental crossings except when they host a Dirac line (Sec. IX). The last column points to noteworthy features. Nodal points allowing for irreducible representations of different dimensions are marked by $(*)$ and are discussed in Sec. VE.

\begin{tabular}{|c|c|c|c|c|c|c|}
\hline & SG & Dirac points & Dirac lines & Accidental points & Accidental lines & Notable features \\
\hline 83 & $P 4 / m$ & & & & & \\
\hline 84 & $P 4_{2} / m$ & $\mathrm{Z}, \mathrm{A}$ & & & & \\
\hline 85 & $P 4 / n$ & $\mathrm{X}, \mathrm{A}, \mathrm{M}, \mathrm{R}$ & & $\mathrm{X}-\mathrm{R}$ & & \\
\hline 86 & $P 4_{2} / n$ & $\mathrm{X}, \mathrm{Z}, \mathrm{M}, \mathrm{R}$ & & $\mathrm{X}-\mathrm{R}$ & & \\
\hline 87 & $I 4 / m$ & & & & & \\
\hline 88 & $I 4_{1} / a$ & $\mathrm{X}, \mathrm{M}$ & & $\mathrm{X}-\mathrm{P}$ & & \\
\hline 123 & $\mathrm{P} 4 / \mathrm{mmm}$ & & & & & \\
\hline 124 & $P 4 / m c c$ & $\mathrm{~A}, \mathrm{Z}, \mathrm{R}$ & & Z-R, Z-A, R-A & & \\
\hline 125 & $P 4 / n b m$ & $\mathrm{M}, \mathrm{A}, \mathrm{X}, \mathrm{R}$ & & X-R, X-M, R-A & & \\
\hline 126 & $P 4 / n n c$ & $\mathrm{X}, \mathrm{Z}, \mathrm{A}, \mathrm{R}, \mathrm{M}$ & & X-R, X-M, Z-R, Z-A & & \\
\hline 127 & $P 4 / m b m$ & & $\overline{\mathrm{MX}}, \overline{\mathrm{AM}}, \overline{\mathrm{AR}}$ & & $k_{x}, k_{y}=\pi$ & \\
\hline 128 & $P 4 / m n c$ & $\mathrm{Z}$ & $\overline{\mathrm{AM}}, \overline{\mathrm{MX}}$ & Z-R, Z-A, R-A & $k_{x}, k_{y}=\pi$ & \\
\hline 129 & $P 4 / n m m$ & & $\overline{\mathrm{RX}}, \overline{\mathrm{AM}}$ & & $k_{x}, k_{y}=\pi$ & \\
\hline 130 & $P 4 / n c c$ & $\mathrm{Z}, \mathrm{R}-\mathrm{Z}(4)$ & $\overline{\mathrm{RX}}, \overline{\mathrm{AM}}, \overline{\mathrm{AR}}$ & Z-R, Z-A & $k_{x}, k_{y}=\pi$ & Movable Dirac; eightfold A \\
\hline 131 & $P 4_{2} / m m c$ & $\mathrm{Z}, \mathrm{A}$ & & Z-A & & \\
\hline 132 & $\mathrm{P}_{2} / \mathrm{mcm}$ & $\mathrm{Z}, \mathrm{R}, \mathrm{A}$ & & Z-R, R-A & & \\
\hline 133 & $P 4_{2} / n b c$ & $\mathrm{X}, \mathrm{Z}, \mathrm{A}, \mathrm{R}, \mathrm{M}, \mathrm{A}-\mathrm{M}(4)$ & & X-R, X-M, Z-A, R-A & & Movable Dirac \\
\hline 134 & $P 4_{2} / n n m$ & $\mathrm{X}, \mathrm{Z}, \mathrm{M}, \mathrm{R}$ & & X-R, X-M, Z-R & & \\
\hline 135 & $P 4_{2} / m b c$ & $\mathrm{Z}$ & $\overline{\mathrm{MX}}, \overline{\mathrm{AM}}, \overline{\mathrm{AR}}$ & Z-A & $k_{x}, k_{y}=\pi$ & Eightfold A \\
\hline 136 & $P 4_{2} / m n m$ & $\mathrm{Z}$ & $\overline{\mathrm{MX}}, \overline{\mathrm{AM}}$ & Z-R & $k_{x}, k_{y}=\pi$ & \\
\hline 137 & $P 4_{2} / n m c$ & $\mathrm{Z}$ & $\overline{\mathrm{AM}}, \overline{\mathrm{RX}}$ & Z-A & $k_{x}, k_{y}=\pi$ & \\
\hline 138 & $\mathrm{P}_{2} / \mathrm{ncm}$ & $\mathrm{Z}, \mathrm{R}-\mathrm{Z}(4)$ & $\overline{\mathrm{AR}}, \overline{\mathrm{AM}}, \overline{\mathrm{RX}}$ & Z-R & $k_{x}, k_{y}=\pi$ & Movable Dirac \\
\hline 139 & $I 4 / \mathrm{mmm}$ & & & & & \\
\hline 140 & $I 4 / \mathrm{mcm}$ & $\mathrm{N}, \mathrm{P}$ & & $\mathrm{N}-\mathrm{P}$ & & \\
\hline 141 & I4/amd & $\mathrm{X}, \mathrm{M}$ & & X-P, X-M & & \\
\hline 142 & $I 4_{1} /$ acd & $\mathrm{X}, \mathrm{M}, \mathrm{N}, \mathrm{P}(*)$ & & X-P, X-M, N-P & & Dirac at $\mathrm{P}(*)$ \\
\hline
\end{tabular}

respectively. Depending on the cell metrics, the Brillouin zone for body-centered tetragonal crystals is one of two distinct polyhedra: an elongated dodecahedron $\left(\mathrm{BCT}_{1}\right.$, for $\left.c<a\right)$ or a truncated square bipyramid $\left(\mathrm{BCT}_{2}\right.$, for $\left.c>a\right)$ [48].

\section{A. Conventions}

The discussion of enforced band features necessitates the frequent use of space-group (SG) symmetries. For the tetragonal SGs the relevant symmetries are twofold and fourfold rotations and mirror symmetries. We define the abbreviations for symmetries as $N_{x y z}(a b c)$, where $N=2,4$ refers to an $N$-fold rotation around the axis given by the vector $(x, y, z)$ followed by a (fractional) lattice translation $(a, b, c)$. For mirror symmetries the notation $M_{x y z}(a b c)$ declares $(x, y, z)$ to be the normal direction of the mirror plane and $(a, b, c)$ again the translation. All symbols implicitly contain also the action on the electron spin. Combined with the spatial action the symmetries can be exemplarily defined as

$$
2_{001}(a, b, c):(x, y, z) \rightarrow(-x+a,-y+b, z+c) \otimes i \sigma_{z},
$$

$4_{001}(a, b, c):(x, y, z) \rightarrow(-y+a, x+b, z+c) \otimes \frac{\sigma_{0}+i \sigma_{z}}{\sqrt{2}}$,

$$
M_{001}(a, b, c):(x, y, z) \rightarrow(x+a, y+b,-z+c) \otimes i \sigma_{z},
$$

where $\sigma_{x}, \sigma_{y}, \sigma_{z}$ refers to the Pauli matrices and $\sigma_{0}$ is the $2 \times 2$ unit matrix. We denote pure translations by a vector $(a, b, c)$ with $t(a, b, c)$.

Additionally, we will make frequent use of time-reversal symmetry $\mathcal{T}=\mathbb{1} \otimes \mathrm{i} \sigma_{y} \mathcal{K}$, which consists of a unitary part acting on spin space and complex conjugation $\mathcal{K}$, whose action in momentum space is $\mathcal{T} \mathbf{k}=-\mathbf{k}$.

It is sufficient to study points, line segments, and planes of one eighth of the full BZ, because all space groups we consider in this work contain time reversal $\mathcal{T}$ and the fourfold rotation symmetry $4_{001}$. Therefore, the band structure and any topological features are always related between different parts of the BZ and one octant of the full BZ suffices. Without loss of generality, we set the lattice constants $a=c=1$.

A time-reversal invariant momentum (TRIM) is an important point of interest for any space-group symmetry analysis. It is defined as a point $\boldsymbol{k}_{\mathrm{TRIM}}$ of the BZ, which is left invariant by the action of time reversal, i.e., $\mathcal{T} \boldsymbol{k}_{\mathrm{TRIM}}=\boldsymbol{k}_{\mathrm{TRIM}}+\boldsymbol{K}$, where $\boldsymbol{K}$ is a reciprocal lattice vector. By describing the reciprocal lattice with coordinates given in its primitive basis, 
(a)

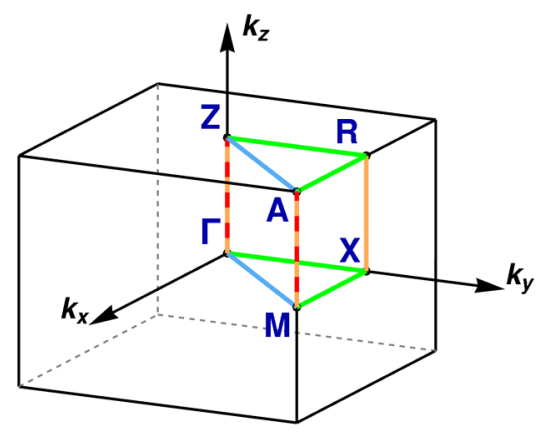

(b)

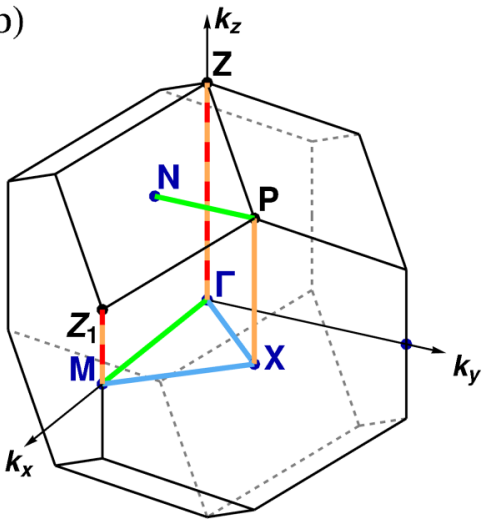

(c)

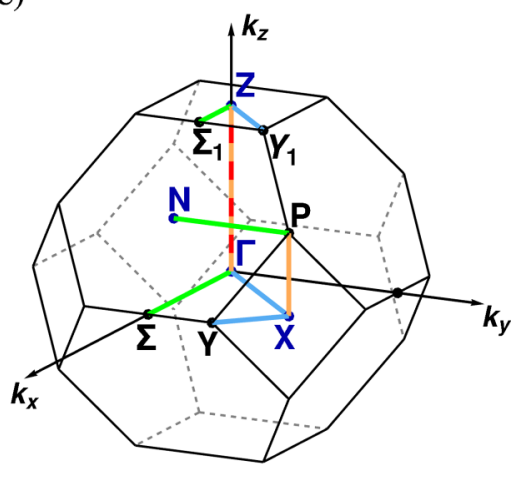

FIG. 1. Brillouin zones of the tetragonal crystal system. TRIMs are labeled in dark blue. Lines of high symmetry, i.e., sections of rotation axes, are highlighted for the fourfold rotation (red) along [001], and the twofold rotations along $\langle 100\rangle$ (green), $\langle 110\rangle$ (light blue), and [001] (orange). (a) Primitive BZ. (b) Body-centered BZ for $c<a, \mathrm{BCT}_{1}$. (c) Body-centered BZ for $c>a, \mathrm{BCT}_{2}$.

it is evident that there are always eight TRIMs in the Brillouin zone. To be specific in the primitive basis the TRIMs are $(0,0,0),\left(\frac{1}{2}, 0,0\right),\left(0, \frac{1}{2}, 0\right),\left(0,0, \frac{1}{2}\right),\left(\frac{1}{2}, \frac{1}{2}, 0\right),\left(\frac{1}{2}, 0, \frac{1}{2}\right)$, $\left(\frac{1}{2}, \frac{1}{2}, 0\right),\left(\frac{1}{2}, \frac{1}{2}, \frac{1}{2}\right)$ for any unit-cell type, i.e., primitive as well as body-centered cells. Keeping this result in mind we describe the Brillouin zone in Cartesian coordinates, which makes the possible symmetries more obvious. Hereby, the primitive and body-centered Brillouin zones must be distinguished (see Fig. 1). Adopting this description, the eight TRIMs for the primitive cell are $\Gamma(0,0,0), \mathrm{X}(0, \pi, 0)(2)$, $\mathrm{Z}(0,0, \pi), \mathrm{R}(0, \pi, \pi)(2), \mathrm{M}(\pi, \pi, 0), \mathrm{A}(\pi, \pi, \pi)$, where (2) denotes TRIMs that appear in two distinct copies related by symmetry. For the body-centered Brillouin zone $\mathrm{BCT}_{1}$ the TRIMs are $\Gamma(0,0,0), \mathrm{X}(\pi, \pi, 0)(2), \mathrm{M}(2 \pi, 0,0)$, $\mathrm{N}(\pi, 0, \pi)(4)$ and for $\mathrm{BCT}_{2} \mathrm{M}(2 \pi, 0,0)$ is replaced by $\mathrm{Z}(0,0,2 \pi)$. Without loss of generality we use the labels of $\mathrm{BCT}_{1}$ unless a material realization requires $\mathrm{BCT}_{2}$.

The point $\mathrm{P}=(\pi, \pi, \pi)$ is not a TRIM of the bodycentered BZ. Yet, it is invariant under the combination of time-reversal symmetry $\mathcal{T}$ with fourfold rotation $4_{001}$. As we will discuss below in Sec. III B, there is a version of Kramers theorem with this combined symmetry leading to nodal points at P. Hereby a prerequisite of Kramers theorem can become dependent on the eigenvalues at $\mathrm{P}$ for the twofold rotation.

To denote segments of high-symmetry lines we use two or more points on the line connected by a hyphen. The shortest connection between the points defines the line. The notation with a hyphen is used, when any point on the high-symmetry line may be the locus of a feature of interest and also when the whole line exhibits a property, e.g., $\Gamma-\mathrm{Z}$ in SG 76 contains movable Weyl points and forms accordion states. For clarity we denote the fourfold rotation axis $\left(0,0, k_{z}\right)$ of the body-centered cell as $\Gamma$-Z-M. If a feature appears for every single point on a line, we use the same points but with an overline, e.g., $\overline{\mathrm{MA}}$ for SG 113 is a line of fourfold degenerate points.

The focus of this work lies on enforced features of the band structure. We distinguish symmetry-enforced properties, which must occur based on symmetry arguments for any realization of a space group with spin degrees of freedom, from accidental features that may exist but depend on details of the system. For example, consider for now $\Gamma-\mathrm{X}$ to be a twofold screw rotation axis. There, all bands can be labeled by one out of two symmetry eigenvalues. We will show that each band must exchange its eigenvalue at least once forming an enforced crossing on the line $\Gamma$-X (cf. Sec. IV A). Furthermore, any crossing of bands with different eigenvalues must be gapless because any term introduced to gap the crossing necessarily breaks the symmetry. It is possible that the bands exchange several times leading to what we refer to as accidental band crossings. Nevertheless, they are symmetry protected like the enforced crossings. Note, if both occur it is generally not possible to label one of them as enforced. This notion of accidental crossings includes nonguaranteed band crossings that are protected by other means than symmetry eigenvalues, e.g., Weyl points situated away from high-symmetry points or lines, which are protected only by translation symmetry and their inherent nonzero Chern number. In other words, we regard enforced features to be stable under arbitrary symmetry-preserving perturbations. They are unaffected by the details of a respective realization.

When naming pointlike topological band crossings, we call fourfold crossings Dirac points only in the presence of inversion symmetry. We regard a point crossing as a species of Weyl point if and only if it carries a nonzero chirality. By default, such a Weyl point is twofold degenerate with a chirality $\mathcal{C}=1$, double Weyl points are twofold degenerate with a chirality of 2 . We refer to fourfold double Weyl points, when discussing two superimposed regular Weyl points with a total chirality of $\mathcal{C}=2$, which is also called a doubled spin- $\frac{1}{2}$ fermion in the literature [49]. We further discuss the fourfold quadruple Weyl point, which consists of two superimposed regular double Weyl points and carries a total charge of $\mathcal{C}=4$ (see Sec. IV E).

\section{B. Systematic search for example materials}

In the following sections, we will identify and discuss the tetragonal SGs, whose nonsymmorphic symmetries enforce various topological and trivial degeneracies on points, lines, and planes. For each of those SGs, we then perform a database search for material examples among the subset of ordered, 


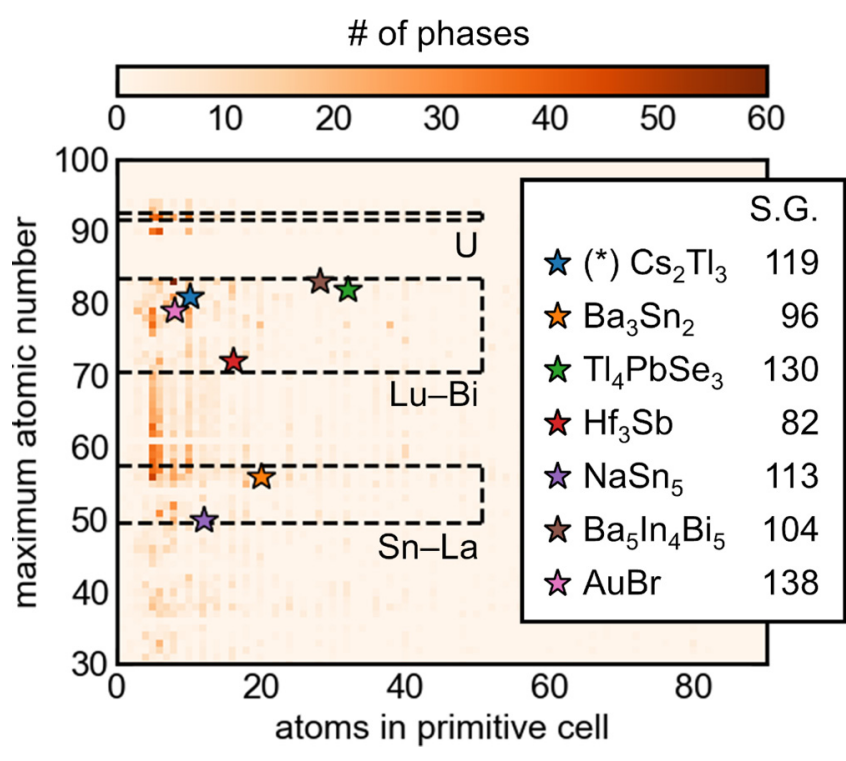

FIG. 2. Distribution of ordered, tetragonal phases in the Materials Project and ICSD (MP $\cap$ ICSD, $N=7039$ ) by atomic number of the heaviest element and the number of atoms in the primitive unit cell. Seven example phases discussed in the text are highlighted. The regions bounded by black dashed lines indicate two of the automated screening criteria explained in the text. $(*) \mathrm{Cs}_{2} \mathrm{Tl}_{3}$ is a hypothetical phase (see text).

inorganic, crystalline phases in the Materials Project database [50,51] that correspond to entries in the Inorganic Crystal Structure Database (ICSD) [52]. The distribution of these $\sim 7000$ tetragonal phases by maximum atomic number and number of atoms in the primitive cell is given in Fig. 2. To identify materials with strong spin-orbit coupling, we screen for phases containing elements Sn-La, Lu-Bi, or U (we exclude most rare earths as $f$ electrons are poorly described by single-particle DFT, but compounds of $\mathrm{La}^{3+}, \mathrm{Lu}^{3+}$, or $\mathrm{U}^{6+}$ have empty or filled $f$ shells). We apply further screens based on the number of atoms in the unit cell (as larger cells have more bands and typically more limited bandwidths), thermodynamic stability (formation energy $\leqslant 50 \mathrm{meV}$ atom $^{-1}$ off the convex hull), and band gap ( $\leqslant 1 \mathrm{eV}$ by a generalized gradient approximation functional). These criteria yield between zero and several dozen phases for each of the space groups of greatest interest. Seven example materials, annotated in Fig. 2, are discussed in detail in the corresponding sections. AuBr and $\mathrm{Cs}_{2} \mathrm{Tl}_{3}$ were added manually to this group, as $\mathrm{AuBr}$ has a band gap wider than the screening criterion, and $\mathrm{Cs}_{2} \mathrm{Tl}_{3}$ is a hypothetical heavier analog of the known $\mathrm{Cs}_{2} \mathrm{In}_{3}$.

For the materials of interest we perform DFT bandstructure calculations with the VASP code [53,54], using the projector augmented-wave (PAW) method $[55,56]$ and the PBE [57] exchange-correlation functional. The relaxed structures stored in the Materials Project are used directly for all phases except hypothetical $\mathrm{Cs}_{2} \mathrm{Tl}_{3}$, whose structure is determined by relaxing Tl-substituted $\mathrm{Cs}_{2} \mathrm{In}_{3}$.

In the main text we will focus on the band dispersion around the features of interest. The complete band structures along the high-symmetry lines of the full $\mathrm{BZ}$ are presented in Appendix A.

\section{APPLICATIONS OF KRAMERS THEOREM}

Kramers theorem states that an antiunitary operation $\mathcal{T}$, e.g., time reversal, with $\mathcal{T}^{2}=-1$ leads to twofold degenerate bands at each invariant momentum [58]. These Kramers-Weyl points were discussed for chiral space groups [59]. We extend the argument to groups containing mirror and rotoinversion symmetries and find a minimal number of paired Weyl points in SGs 119 and 120. It is favorable to obtain simple realizations of Weyl semimetals comprising few Weyl points with a large separation to simplify further analysis. In such systems the signatures of Weyl points are more prominent, e.g., in transport properties and as a less complex arrangement of the surface Fermi arcs [60-62].

Beyond this well-known application of Kramers theorem at TRIMs, it also leads to other topological features and can be adapted to convey insights into the tabulated irreducible group representations $[63,64]$. Among these features there are nodal planes as a result of twofold screw rotations, which we discuss separately in Sec. VIII, as well as nodal lines on highsymmetry axes following an analogous argument with glide mirror symmetries and rotoinversions.

We first discuss Kramers-Weyl points at TRIMs and then elaborate on how Kramers pairing occurs away from TRIMs. For the second part the combination of time-reversal with (non)symmorphic symmetries results in line and point degeneracies.

\section{A. Kramers-Weyl points}

Weyl points are, in their basic form, twofold degenerate crossings acting as sources and sinks of Berry curvature, which is measured by their topological charge, the chirality $\mathcal{C}$. It corresponds to the Chern number calculated on an enclosing two-dimensional manifold, e.g., a sphere. Since the Berry curvature transforms as a pseudovector under reflections, one derives that the chirality of any nodal feature on a mirror plane must be equal to its negative and thereby vanish. By excluding point groups with mirror and inversion symmetry it is possible to conclude that any given TRIM carries twofold degenerate nodal points with nonzero chirality dubbed as Kramers-Weyl points [59].

Yet, it is insufficient to consider only chiral crystals, meaning crystals with rotations and translations only, because a mirror symmetry can coexist with a Weyl point as long as the Weyl point is not situated on the mirror plane. In that case, the mirror symmetry relates two Weyl points of opposite chirality. The tetragonal SGs 111-114 and 119-122 contain such enforced topological crossings. To aid the search of new materials with few Weyl points, we have listed the multiplicities of enforced Weyl points in the column "No. Weyl" in Table I. Depending on the considered band index, different multiplicities appear and are listed in ascending order. Note that this column contains all charged nodal points, not just (Kramers-)Weyl points, independently of the magnitude of their charge. The SGs $99-110,115-118$ each contain a version of $M_{100}$, which has mirror planes intersecting all TRIMs, and also do not contain Weyl points enforced by other means, thus leaving the entry in column "No. Weyl" empty. Although each space group without mirror symmetries contains in principle a set of eight Kramers-Weyl points, nodal lines or planes may 
lead to extended instead of pointlike degeneracies. Consider SG 81 with its fourfold rotoinversion, where the lines $\Gamma-Z$ and M-A exhibit only twofold representations, such that just $X$ and $\mathrm{R}$ remain as Kramers-Weyl points yielding a total multiplicity of 4.

Finally, we find the minimal number of one Weyl point pair in SG 119 by choosing a space group with a specific mirror symmetry and a body-centered unit cell. Consider SG 82 as starting point, a body-centered version of SG 81, which is generated by a fourfold rotoinversion. Analogous to SG 81 the twofold degenerate line $\Gamma-\mathrm{Z}-\mathrm{M}$ reduces the number of TRIMs with Kramers-Weyl points, leaving six Weyl points at $\mathrm{X}$ and $\mathrm{N}$. The rotoinversion relates Weyl points of opposite chirality, i.e., the nodal points $(\pi, \pi, 0)$ and $(\pi,-\pi, 0)$ both labeled X, and likewise for the crossings at N. If the mirror symmetry $M_{100}$ is added to remove the Weyl points at $\mathrm{N}$ as well, the resulting group is SG 119. For SG 119 the twofold degeneracies at $\mathrm{N}$ are part of almost movable nodal lines (see Sec. VI A) and carry no chirality. Hence, SG 119 yields two enforced Weyl points at $\mathrm{X}$ related by an improper rotation and a mirror symmetry, which achieves the minimal number of one pair of Weyl points on a lattice [46,47]. For SG 120 a single pair of Kramers-Weyl points follows from an identical argument. Even though these two SGs have only two Weyl points, this will generally not lead to Fermi arcs that are well separated from bulk bands in the entire surface BZ. This is because the TRIMs without Weyl points in SGs 119 and 120 are part of nodal lines, leading to Fermi surface pockets that partially overlap with the Fermi arcs in the surface spectra (cf. Fig. 14 and Appendix B 1).

The presence of only two Weyl points in SGs 119 and 120 per band pair is at odds with the common notion that four Weyl points are the minimal number achievable in a system with time-reversal symmetry [60-62]. The argument assumes that time reversal relates distinct Weyl points with the same chirality and concludes by the Nielsen-Ninomiya theorem that two additional Weyl points of opposite chirality must exist $[46,47]$. Although for a Weyl point at a TRIM the first assumption is false, the minimal number of four seems unchallenged since there are eight TRIMs. But, as we have noted above, due the mirror symmetry $M_{100}$ in the body-centered SGs 119 and 120 all but two nodal points have vanishing chirality. Note, however, that it is possible to obtain a single Weyl point if the nodal manifold of opposite chirality appears in the shape of a topological nodal plane instead of a Weyl point (see Sec. VIII).

To further study SG 119 we have created a minimal model, confirming that the chiralities of Weyl points at $\mathrm{X}$ are indeed +1 and -1 , and determined the surface states (see Appendix B 1). Here we want to highlight one property of the surface states for SG 119. We find that each surface exhibits two Fermi arcs connecting the projections of the two $\mathrm{X}$ points in the surface BZ. The arcs are related by time-reversal symmetry and appear in disjoint bulk band gaps (see Fig. 14).

Nevertheless, it is unusual that two arcs appear if there is only one pair of singly charged Weyl points. To understand the relation of Weyl points and Fermi arcs, the standard approach is to consider gapped planar subsystems on which one may calculate the Chern number. It is a peculiarity of the bodycentered BZ [see Figs. 1(b) and 1(c)], that an oriented plane normal to $(1,1,0)$ that passes for example the point $\left(\frac{\pi}{2}, \frac{\pi}{2}, 0\right)$ will also include the point $\left(-\frac{\pi}{2},-\frac{\pi}{2}, 0\right)$. Hence, considering the orientation of the normal vector at the two exemplary points the plane does not capture the chirality of one of the Kramers-Weyl points at X. The plane has Chern number zero. If then a slab is made, i.e., the periodic boundary conditions are lifted, such that this plane is truncated, the subsystem of the plane appears as two parallel lines in the surface BZ. We find indeed that the surface states pierce this line twice but in opposing directions, which agrees with the vanishing Chern number of the corresponding subsystem.

One concludes that considering a gapped and planar subsystem is not sufficient to discern the nontrivial topology of SG 119 and analogously of SG 120. Rather, it is the chiralities of the Weyl points alone that indicate the presence of topological surface states and the connectivity of the body-centered BZ allows for two Fermi arcs even for singly charged Weyl points.

\section{Material example: $\mathrm{Hf}_{3} \mathrm{Sb}$}

As an example for SG $82(I \overline{4})$, which enforces only six Kramers-Weyl points per pair of bands, we present the band structure of $\mathrm{Hf}_{3} \mathrm{Sb}$ in Fig. 3(a). It crystallizes with $c<a$ $[65,66]$, i.e., its $\mathrm{BZ}$ is of type $\mathrm{BCT}_{1}$. Kramers-Weyl points appear at $\mathrm{N}$ and $\mathrm{X}$ for all bands, whereas the points $\Gamma$ and $\mathrm{M}$ are part of a nodal line. Accidental band crossings occur for several bands along the twofold rotation axis X-P.

\section{Material example: $\mathrm{Cs}_{2} \mathrm{Tl}_{3}$}

$\mathrm{Cs}_{2} \mathrm{Tl}_{3}$ is a hypothetical, heavier analog to $\mathrm{Cs}_{2} \mathrm{In}_{3}$ in $\mathrm{SG}$ $119(I \overline{4} m 2)$ with $c>a$ [67]. We find the formation energy of $\mathrm{Cs}_{2} \mathrm{Tl}_{3}$ with respect to the elements is favorable, but the compound is not among the reported binaries in the Cs-Tl system [68-70]. The band structure shown in Fig. 3(b) shows the two distinct Kramers-Weyl points at X. The nodal crossings at $\mathrm{N}$ are also enforced by Kramers theorem, but they are part of nodal lines within mirror planes and thus without chirality. Similarly, the TRIMs $\Gamma$ and $\mathrm{Z}$ do not exhibit a nodal point because they are part of a nodal line.

\section{B. Kramers theorem beyond TRIMs}

In this section we discuss band degeneracies due to (non)symmorphic, antiunitary symmetries. It is instructive to consider for a start symmorphic symmetries combined with the time-reversal operation $\mathcal{T}$ in the context of Kramers theorem. The best-known case is inversion symmetry $\mathcal{P}$. The combination $\mathcal{P} \mathcal{T}$ enforces twofold degeneracies at all momenta because $(\mathcal{P} \mathcal{T})^{2}=-1$ and all $k$ are invariant momenta. Due to this ubiquitous twofold degeneracy in centrosymmetric groups, we have split the results of this paper into the cases without and with inversion symmetry (see Tables I and II).

The combination of time reversal with nonsymmorphic symmetries can lead to nodal lines and planes, which occur at invariant $k$ points where the compound symmetry squares to any number different from 1 (see Sec. VIII). In the following paragraphs we describe variations of this argument, which lead to topological band crossings. First we discuss symmorphic antiunitary compound symmetries, which unlike 
(a)

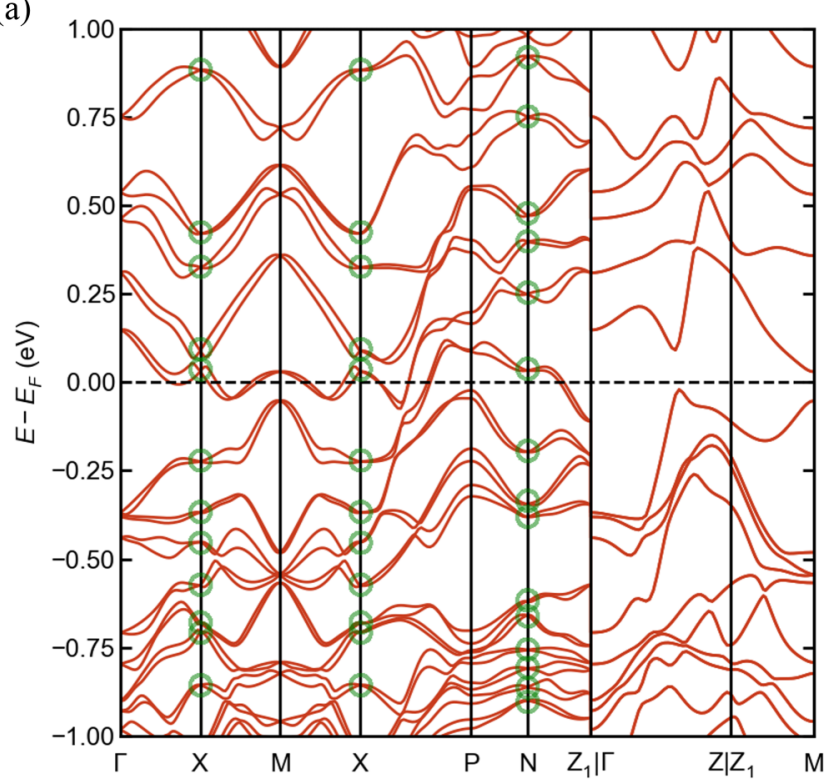

(b)

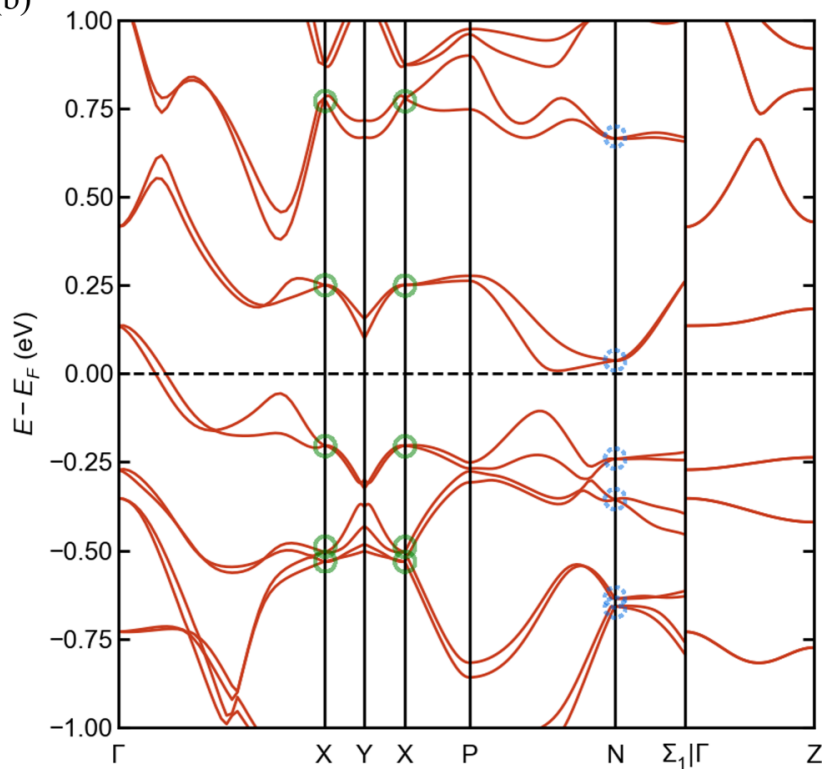

FIG. 3. Kramers-Weyl realization. Green circles mark the Weyl points at TRIMs. (a) Band structure of $\mathrm{Hf}_{3} \mathrm{Sb}$ (SG 82). Each pair of bands exhibits six independent Weyl points. (b) Band structure of $\mathrm{Cs}_{2} \mathrm{Tl}_{3}$ (SG 119). Only two Weyl points per pair of bands are enforced. Dashed blue circles highlight crossings at $\mathrm{N}$ which are part of almost movable nodal lines in mirror planes and contribute no chirality see (Sec. VI A).

$\mathcal{P} \mathcal{T}$ do not square to the -1 but still yield nodal lines or points and then continue to nonsymmorphic operations responsible for Kramers pairing restricted to certain rotation eigenvalues. Both arguments enforce Weyl points, which are not situated at TRIMs but pinned to the point $P$.

\section{Symmorphic antiunitary symmetries}

Consider the noncentrosymmetric SGs 81, 82 and SG 119 from the previous section, which exhibit a twofold degenerate nodal line along $\Gamma-\mathrm{Z}$ and $\Gamma-\mathrm{Z}-\mathrm{A}$, respectively. This degeneracy can be understood with the symmorphic operation $\overline{4}_{001} \mathcal{T}$, where the rotoinversion $\overline{4}_{001}$ acts on the fourfold rotation axes like inversion by itself. There is an analogy to the case of $\mathcal{P} \mathcal{T}$ symmetry even though $\left(\overline{4}_{001} \mathcal{T}\right)^{2} \neq-1$. As $\overline{4}_{001} \mathcal{T}$ leaves $\mathbf{k}$ invariant, for the moment one may assume that $\overline{4}_{001} \mathcal{T}$ creates a linearly dependent state $\overline{4}_{001} \mathcal{T}\left|\psi_{\mathbf{k}}\right\rangle=\exp (i \varphi)\left|\psi_{\mathbf{k}}\right\rangle$, when acting on the eigenstate $\left|\psi_{\mathbf{k}}\right\rangle$ of a Hamiltonian by producing only a phase factor $\exp (i \varphi)$. By substituting this equation in the form $\left|\psi_{\mathbf{k}}\right\rangle=\exp (-i \varphi) \overline{4}_{001} \mathcal{T}\left|\psi_{\mathbf{k}}\right\rangle$ four times into itself, a contradiction $\left|\psi_{\mathbf{k}}\right\rangle=\left(\overline{4}_{001} \mathcal{T}\right)^{4}\left|\psi_{\mathbf{k}}\right\rangle=-\left|\psi_{\mathbf{k}}\right\rangle$ is found by using $\mathcal{T}^{2}=-1$ with $\overline{4}_{001}^{4}=-1$ for the last step. Hence, the assumption is wrong and $\overline{4}_{001} \mathcal{T}$ must relate the eigenstate $\left|\psi_{\mathbf{k}}\right\rangle$ to a linearly independent state $\overline{4}_{001} \mathcal{T}\left|\psi_{\mathbf{k}}\right\rangle$ of the same energy.

The same argument can be used as well at TRIMs for the operation $4_{001} \mathcal{T}$ comprising the symmorphic proper rotation $4_{001}$. But, more intriguingly for body-centered space groups the symmetry $4_{001} \mathcal{T}$ leaves another momentum invariant, the point $\mathrm{P}$. By the same argument as above, $\mathrm{P}$ will always be degenerate. We highlight Weyl points at $\mathrm{P}$ in the last column of Table I because they add to the number of Kramers-Weyl points without being at a TRIM.

\section{Eigenvalue-dependent Kramers theorem}

Here we show a variant of Kramers theorem that only applies to states with certain symmetry eigenvalues, which we refer to as the eigenvalue-dependent Kramers theorem. To illustrate this term, note that typically Kramers theorem pairs bands differently depending on whether symmetry eigenvalues are complex or real. In that case, a band with a real eigenvalue is paired to a band with the same eigenvalue, whereas a complex eigenvalue is paired to its complex conjugate. But for the eigenvalue-dependent Kramers theorem only some symmetry eigenvalues are paired, whereas for others the theorem does not hold at all and no pairing is obtained. It is a subtle and common feature of many band structures that all bands at a given high-symmetry point of reciprocal space have the same degeneracy. But, this is not always true. The tetragonal SGs 80, 92, 96, 98, 109, and 142 have irreducible representations of different dimension at high-symmetry points. This can be understood with the eigenvalue-dependent Kramers theorem. To do so, we revisit the above observation that $\left(\overline{4}_{001} \mathcal{T}\right)^{2} \neq-1$ for nonsymmorphic rotations as a starting point for the theorem.

The presence of irreducible representations of unequal dimensions at the same point can be explained by considering a time-reversal-containing symmetry, which fulfills Kramers theorem only for certain eigenvalues. To illustrate this, consider the proof [58] of Kramers theorem for a state $|\psi\rangle$ and an antiunitary operation $\tilde{\mathcal{T}}$ fulfilling $\tilde{\mathcal{T}}^{2}|\psi\rangle=a|\psi\rangle$ with $a \in \mathbb{C}$ :

$$
\langle\psi \mid \tilde{\mathcal{T}} \psi\rangle=\left\langle\tilde{\mathcal{T}}^{2} \psi \mid \tilde{\mathcal{T}} \psi\right\rangle=a^{*}\langle\psi \mid \tilde{\mathcal{T}} \psi\rangle,
$$

where we used the property $\langle\psi \mid \phi\rangle=\langle\tilde{\mathcal{T}} \phi \mid \tilde{\mathcal{T}} \psi\rangle$ of antiunitary operators. $\tilde{\mathcal{T}}|\psi\rangle$ is an orthogonal state to $|\psi\rangle$ if $a \neq 1$. The conventional time reversal yields $a=-1$ leaving no room for a dependence on eigenvalues of $\psi$. If we consider the antiunitary symmetry $\tilde{\mathcal{T}}=\mathcal{T} 4_{001}(a, b, c)$ at an invariant $k$ point, then $a=-\alpha_{2_{001}}$ depends on the rotation eigenvalues $\alpha_{2_{001}}$ of the twofold rotation symmetry resulting from $\left(4_{001}(a, b, c)\right)^{2}$. 
For $c=\frac{1}{4}$ or $\frac{3}{4}$ the two eigenvalues differ at $k_{z}=\pi$ by a factor of -1 . Given that, we can conclude that one but not the other eigenstate of $2_{001}(a-b, b+a, 2 c)$ will have a Kramers partner. In other words, only a band with twofold rotation eigenvalue +1 but not -1 has a Kramers partner.

The SGs 80, 92, 96, 98, 109, and 142 contain highsymmetry points hosting irreducible representations of different dimensions due to eigenvalue-dependent pairing. We highlight these features in Tables I and II with the asterisk symbol (*) next to the label of the high-symmetry point, which can be found either in the fourth or the last column.

This eigenvalue-dependent Kramers theorem makes a difference at points in the $\mathrm{BZ}$ with $k_{z}=\pi$ that are invariant under $\tilde{\mathcal{T}}$, i.e., where one eigenvalue of the twofold screw rotation is $\alpha_{2001}=-1$. These are the points $\mathrm{Z}$ and $\mathrm{A}$ in the primitive unit cell and $\mathrm{P}$ for the body-centered case. Note that at TRIMs the degeneracy will not always be increased because time reversal $\mathcal{T}$ and $\tilde{\mathcal{T}}$ can relate the same symmetry eigenvalues. For them to act differently on 2001 eigenvalues it is necessary that the fourfold rotation contains a partial lattice translation perpendicular to the rotation axis, as is the case in SGs 92 and 96 (see the point A in the example in Sec. IV C). Similarly, additional symmetries can equalize the number of degenerate bands. Compare the unequal dimension of representations at $P$ of SG 109 with the uniformly twofold bands at P for SG 110. The latter is due to a line of Kramers degeneracies caused by $M_{010}\left(0,0, \frac{1}{2}\right) \mathcal{T}$ [ cf. Fig. 11(b)] along the path N-P.

Beyond the tetragonal SGs this argument can be extended to a few hexagonal and cubic cases, which is left for future work.

\section{NONSYMMORPHIC WEYL POINTS}

In this section we discuss how screw-rotation symmetries in tetragonal SGs lead to protected Weyl points. That is, we show how the momentum dependence of the symmetry eigenvalues enforces band crossings along a line in the BZ. Weyl points in tetragonal SGs come in four different varieties: single Weyl points, double Weyl points, fourfold double Weyl points, and fourfold quadruple Weyl points.

\section{A. Hourglass and accordion states}

A twofold screw rotation consists of a twofold rotation followed by half a lattice translation along the rotation axis, e.g., $2_{001}\left(a, b, \frac{1}{2}\right)$ for arbitrary lateral translation components $a$ and $b$. These symmetries square to a full lattice translation and acquire a negative sign from the spin component, which we denote as $-t(0,0,1)$ for the example above. Translation eigenvalues are given by a phase factor, defined through the crystal momentum $\mathbf{k}$. The eigenvalues of the screw rotation are therefore restricted to the two square roots $\pm \sqrt{-e^{i k_{z}}}$ in the example above, where the rotation axis is in the $z$ direction and we will label them with their sign. Evaluated at the timereversal invariant momenta on the rotation axis, these are $\pm i$ and \pm 1 for $k_{z}=0$ and $\pi$, respectively, corresponding to $\Gamma(\mathrm{M})$ and $Z$ (A). Time-reversal symmetry ensures a Kramers partner at the same energy with the complex-conjugate eigenvalue, i.e., it creates pairs $(+,-)$ at $k_{z}=0$ and two pairs $(+,+)$ and $(-,-)$ at $k_{z}=\pi$. The bands connecting these degenerate

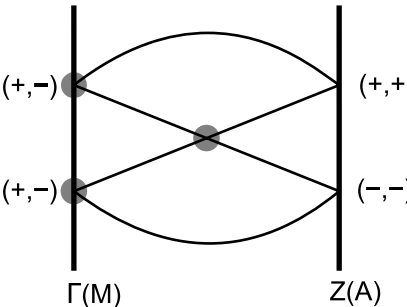

(a) $2001\left(a, b, \frac{1}{2}\right)$
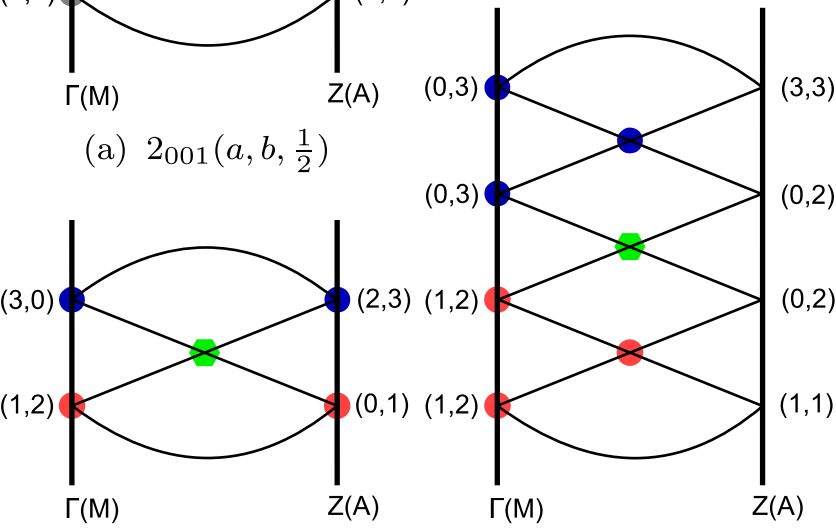

(b) $4001\left(a, b, \frac{1}{2}\right)$

(c) $4001\left(a, b, \frac{1}{4}\right)$

FIG. 4. Connectivity diagrams with minimal band crossings for the screw rotations found in tetragonal space groups. The case for a fourfold screw rotation with $n=3$ looks like $n=1$ with redefined labels. The colored symbols indicate the chiral charge of each crossing. Blue and red dots mark chirality +1 and -1 , respectively, gray dots a chirality of $|\mathcal{C}|=1$ with undetermined sign, and green hexagons indicate a double Weyl point with $\mathcal{C}= \pm 2$.

points must necessarily cross at some point on the axis and this band crossing is protected by the different symmetry eigenvalues. On the axes left invariant by the rotation, the total dispersion of the four bands involved creates an hourglass shape [71] as shown in Fig. 4(a).

Combining a twofold screw rotation with time-reversal symmetry results in an additional antiunitary symmetry squaring to a full lattice translation. Invariant $k$ points are restricted to two planes perpendicular to the rotation axis $\hat{\mathbf{e}}_{i}$, with $\mathbf{k} \hat{\mathbf{e}}_{i}=$ 0 and $\pi$. On the latter plane, this symmetry squares to a lattice translation with eigenvalue $e^{i \mathbf{k} \hat{\mathbf{e}}_{i}}=-1$ and enforces Kramers degenerate states in the whole plane. Hence, the degeneracies at $\mathrm{Z}$ (A) in the band connectivity diagrams are part of these nodal planes and do not form Weyl points. For the topology of such band degeneracies, see the section about topological nodal planes (Sec. VIII).

In a similar manner, we can construct the band connectivity for fourfold nonsymmorphic rotation symmetries $4_{001}\left(a, b, \frac{n}{4}\right)$ as defined in Eq. (2.2). The two invariant axes in primitive lattices are $\Gamma-Z$ and M-A. Together with translations, they generate SGs $76-78$ with $n=1,2,3$, respectively. Where additional symmetries do not impose further degeneracies, these arguments also hold for their supergroups, specifically for SGs 91 and 95 on both invariant lines and for SGs 92 and 96 on $\Gamma-Z$. Applying such a symmetry four times results in a full lattice translation and a minus sign from the spin component $-t(0,0, n)$. The eigenvalues are therefore given by the fourth roots of $-\exp \left(i n k_{z}\right)$, which we label with the integer $p \in\{0,1,2,3\}$, defined through the parametrization

$$
\alpha_{p}=\exp \left[i \frac{\pi}{4}(2 p+1)\right] \exp \left(i \frac{n}{4} k_{z}\right) .
$$




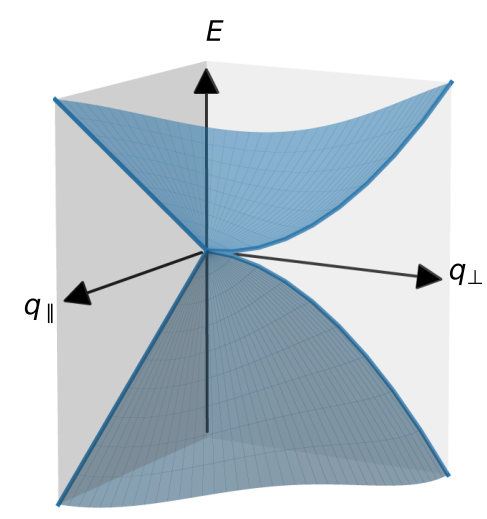

(a) Double Weyl point

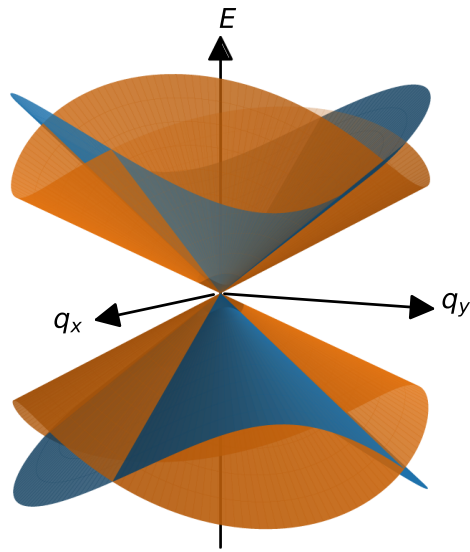

(b) Fourfold double Weyl point

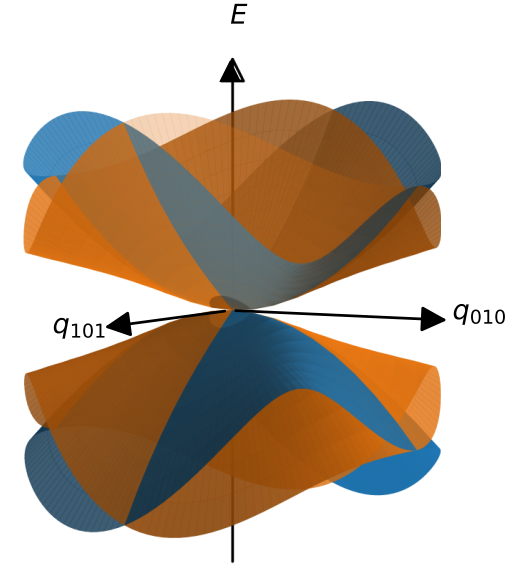

(c) Fourfold quadruple Weyl point

FIG. 5. Dispersion of symmetry-enforced Weyl nodes with Chern number $>1$. The coordinates $q$ in reciprocal space are given relative to the respective nodal point. (a) Double Weyl nodes are twofold degenerate, with linear dispersion along the fourfold rotation axis $q_{\|}$and quadratic dispersion perpendicular to it $q_{\perp}, C_{\mathrm{dW}}= \pm 2$. (b) A fourfold double Weyl point is a fourfold degeneracy with linear dispersion in all directions. Along high-symmetry lines a twofold degeneracy is enforced. The colors mark the two symmetry-related Weyl points. The Chern number is the sum of these two Weyl points $C_{\mathrm{fW}}=2 C_{\text {Weyl }}= \pm 2$. (c) The fourfold double Weyl is built from two symmetry-related double Weyl points of equal chirality, the total Chern number is $C_{\mathrm{fdW}}=2 C_{\mathrm{dW}}= \pm 4$. Twofold-degenerate lines in (b) and (c) are part of nodal planes (see Sec. VIII). The dispersion perpendicular to a fourfold Weyl line of Sec. VII is of the same type as in (b).

At the two TRIMs on any invariant axis, time-reversal symmetry $\mathcal{T}$ pairs bands with complex-conjugate eigenvalues. For $k_{z}=0$, i.e., at $\Gamma$ and $\mathrm{M}$, we find $\alpha_{0}{ }^{*}=\alpha_{3}$ and $\alpha_{1}{ }^{*}=\alpha_{2}$, and we label the degeneracy with $\left(p, p^{\prime}\right)=(0,3)$ and $(1,2)$, respectively. For $k_{z}=\pi$, i.e., at $\mathrm{Z}$ and $\mathrm{A}$, we have to distinguish the three different possible fractional translations.

With $n=1$, we find $\alpha_{0}{ }^{*}=\alpha_{2}=-i$, leading to the pairing $(0,2)$. The two real eigenvalues $\alpha_{1}=-1$ and $\alpha_{3}=1$ get a Kramers partner with the same eigenvalue each, leading to the pairing $(3,3)$ and $(1,1)$. Connecting these pairs creates a minimum of three band crossings on the invariant axis. This pattern is called an accordion state $[43,72]$ and is shown in Fig. 4(c). Each of these crossings is protected by different rotation symmetry eigenvalues. For a screw rotation with $n=3$ the same pattern is found, but with interchanged labels $p \rightarrow(p-1) \bmod 4$.

For $n=2$ there are no real eigenvalues at either TRIM. Whereas the pairing at $\Gamma(\mathrm{M})$ remains unchanged, the pairing at $\mathrm{Z}(\mathrm{A})$ is now $(0,1)$ and $(2,3)$. This allows a simpler band connectivity made up of four bands only and one band crossing along the path [see Fig. 4(b)].

The chirality $C$ of these Weyl points can be inferred from the ratio of eigenvalues involved in a crossing [40]. Twofold screw rotations can create only single Weyl points of chirality \pm 1 , whereas fourfold screw rotations lead to at least one double Weyl point with chirality \pm 2 . Further details of each type of crossing will be discussed below.

\section{B. Single Weyl points}

These are conventional Weyl points with a linear dispersion in each direction and a topological charge of $\mathcal{C}= \pm 1$ [40]. For an hourglass dispersion from a twofold screw rotation, all movable crossings and the ones at $\Gamma$ are of this type. In the accordion dispersion, single Weyl points are found whenever the ratio of rotation eigenvalues in a band crossing is purely complex. In that case, the chirality is completely determined by symmetry eigenvalues

$$
\frac{\alpha_{p}}{\alpha_{p^{\prime}}}= \pm i \Rightarrow \mathcal{C}=\mp 1 \text {. }
$$

Here, $\alpha_{p}$, as defined in Eq. (4.1), is the symmetry eigenvalue of the upper band when moving to larger $k_{z}$ and $\alpha_{p^{\prime}}$ the eigenvalue of the lower band. This implies a sign change when rearranging the order of bands. This condition is always met for the band crossings at $\Gamma$ and for two of the four movable crossings in the accordion state. In both cases, the order in energy might be exchanged, but the sum of all chiralities vanishes.

\section{Double Weyl points}

Double Weyl points are twofold band crossings, where the dispersion to lowest order is linear along one axis and quadratic in directions perpendicular to it [73], shown in Fig. 5(a). The Chern number of a manifold enclosing such a node has absolute value $|\mathcal{C}|=2$. They can be found among the crossings enforced by fourfold screw rotations, where the ratio of rotation eigenvalues of the crossing bands is $\frac{\alpha_{p}}{\alpha_{p^{\prime}}}=-1$. With the parametrization of Eq. (4.1), this is the case for the pairs $\left(p, p^{\prime}\right)=(0,2)$ and $(1,3)$. For all of these, the direction of linear dispersion is along the screw axis and the sign of their topological charge depends on details of the Hamiltonian and is not determined from the order of eigenvalues alone.

\section{Material example: $\mathrm{Ba}_{3} \mathrm{Sn}_{2}$}

Here, we present an example material with Weyl nodal points, whose existence is enforced by screw rotation symmetries. These Weyl points are part of an hourglass or accordion dispersion, as discussed in the previous sections. 
(a)

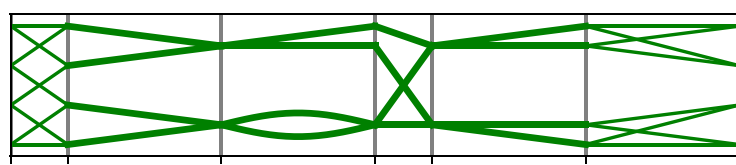

(b)

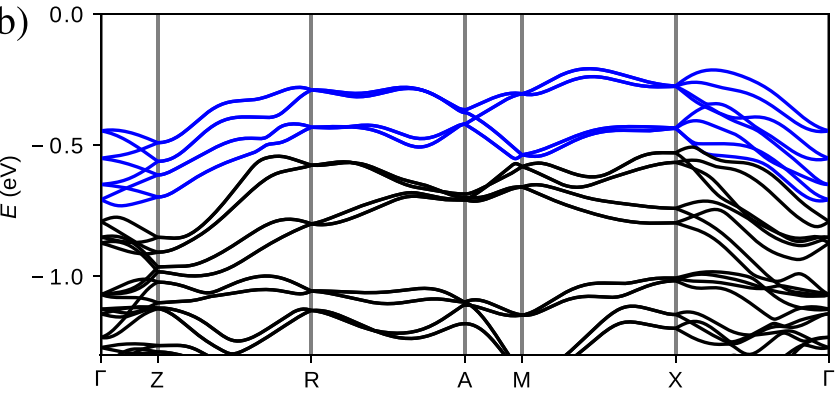

FIG. 6. Band structures for SG 96. (a) Schematic band structure based on the connectivity of irreducible representations. Thick lines indicate twofold degenerate bands within nodal planes. (b) Band structure of $\mathrm{Ba}_{3} \mathrm{Sn}_{2}$ calculated from first principles. The connectivity for all bands is consistent with the above sketch. The accordion states along $\Gamma-Z$ can be seen most clearly in the uppermost eight bands (blue), whereas the hourglass states are only resolved in some bands lower in energy.

The binary compound $\mathrm{Ba}_{3} \mathrm{Sn}_{2}$, which crystallizes in SG No. $96\left(P 4_{3} 2_{1} 2\right)$ [74], is an example of a material with accordion states along the $\Gamma-Z$ line. This material is an electron-precise (insulating) Zintl phase, with a narrow band gap [Fig. 13(f)] and a metallic luster. The $\left[\mathrm{Sn}_{2}\right]^{6-}$ Zintl ion is unlikely to tolerate heavy doping before decomposition, but light heterovalent doping may be possible. The accordion states are shown in Fig. 6 alongside a connectivity diagram for this SG and contains single and double Weyl points as indicated in the accordion states in Fig. 4(c).

Furthermore, this SG has twofold screw rotations with axes along the 100 and 010 directions. As explained above, this enforces an hourglass dispersion along $\Gamma$-X. In the uppermost occupied bands, the movable crossings of the hourglass dispersion are to close to $\mathrm{X}$ to be resolved, but they are visible in the bands below.

An additional feature is the fourfold degenerate band crossing along A-M. This feature is protected by symmetry eigenvalues, but not mandated by band connectivity. It can in principle be removed by exchanging the bands at A such that the fourfold degenerate representation falls between the two twofold representations. In either case, the fourfold degeneracy at half-filling has to carry a Chern number of $\mathcal{C}=\mp 2$ to cancel the topological charges of the double Weyl point with $\mathcal{C}= \pm 2$ in the accordion state (see Sec. IV D about fourfold Weyl nodes below). This SG and example material will also serve as an example in the context of topological nodal planes in Sec. VIII.

\section{Fourfold double Weyl points}

A fourfold double Weyl point is a fourfold, pointlike degeneracy with linear dispersion, that splits into four nondegenerate bands in all directions excluding the twofolddegenerate nodal planes, which are covered in more detail in Sec. VIII. From a topological point of view these degeneracies are two symmetry-related Weyl points of identical chirality on top of each other, leading to a total Chern number of \pm 2 . An exemplary dispersion is shown in Fig. 5(b). Such a feature is enforced by a combination of time reversal and a spatial symmetry which enforces a different Kramers partner than time-reversal symmetry alone.

These conditions are met in the tetragonal crystal system by SGs 90 and 94 at M and A and in SGs 92 and 96 at M. First, we are going to discuss the arguments leading to fourfold double Weyl points, using SG 90 as an example. The TRIMs $\mathrm{M}$ and $\mathrm{A}$ are on an axis left invariant by the fourfold rotation, therefore, we can label the states with their eigenvalues,

$$
4_{001}\left(\frac{1}{2}, \frac{1}{2}, 0\right)|p\rangle=e^{i(2 p+1) \frac{\pi}{4}}|p\rangle,
$$

indexed by $p=0,1,2,3$. Time-reversal symmetry pairs states with complex-conjugate eigenvalues, i.e., $(0,3)$ and $(1,2)$.

Consider now the additional antiunitary symmetry from combining a twofold screw rotation with time-reversal symmetry $2_{010}\left(\frac{1}{2}, \frac{1}{2}, 0\right) \mathcal{T}$. Its invariant points are restricted to the two planes $k_{y}=0$ and $\pi$ where it squares to 1 and -1 , respectively. In the latter case Kramers pairs are enforced, i.e., every state in the plane is twofold degenerate and we call it a nodal plane.

To find the eigenvalue of the Kramers partner, the commutation relation of the fourfold rotation and the antiunitary symmetry is needed. After some algebra, we find

$$
\begin{aligned}
4_{001} & \left(\frac{1}{2}, \frac{1}{2}, 0\right) 2_{010}\left(\frac{1}{2}, \frac{1}{2}, 0\right) \mathcal{T} \\
\quad= & 2_{010}\left(\frac{1}{2}, \frac{1}{2}, 0\right) \mathcal{T} t(1,0,0)\left(-4_{001}\left(\frac{1}{2}, \frac{1}{2}, 0\right)\right)^{3} .
\end{aligned}
$$

Therefore, the Kramers partner $2_{010}\left(\frac{1}{2}, \frac{1}{2}, 0\right) \mathcal{T}|p\rangle$ has the eigenvalue $\alpha_{p^{\prime}}=\left(\alpha_{p}^{3}\right)^{*}=\exp \left[i(2(p+2)+1) \frac{\pi}{4}\right]$, i.e., $p^{\prime}=$ $p+2$ and thus the pairing is $(0,2)$ and $(1,3)$. In combination with the pairings $(0,3)$ and $(1,2)$ from time-reversal symmetry, this establishes the fourfold degeneracy. In other space groups, the translational part of these symmetries might differ, but Eq. (4.4) holds with a modified translation. For all TRIMs, where the eigenvalue of the translation is -1 , the same pairing is found. Alternatively, the presented results can be understood by considering the eigenvalues $\pm i$ of the twofold rotation $2_{001}$. Time reversal $\mathcal{T}$ pairs opposite signs at the TRIMs, whereas $2_{010}\left(\frac{1}{2}, \frac{1}{2}, 0\right) \mathcal{T}$ anticommutes with $2_{001}$ on the line $\mathrm{M}-\mathrm{A}$ and therefore pairs identical eigenvalues.

The fourfold degeneracy splits into nondegenerate bands, except for the $k_{x}=\pi$ and $k_{y}=\pi$ nodal planes. The spectrum is linear to lowest order in $\mathbf{q}=\mathbf{k}-\mathbf{K}_{\text {TRIM }}$ and is made from two Weyl cones, that are related by a $\frac{\pi}{2}$ rotation [see Fig. 5(b)]. A minimal, linearized Hamiltonian for this band crossing has four bands and can always be brought into block-diagonal form, consisting of two Weyl Hamiltonians [49]. Furthermore, $2_{010}\left(\frac{1}{2}, \frac{1}{2}, 0\right) \mathcal{T}$ relates the coefficients of the Kramers-Weyl points such that they have the same chirality. Therefore, the total chirality of the fourfold Weyl point for two occupied bands has to add up to \pm 2 . Hence, they are also referred to as double-spin- $\frac{1}{2}$ Weyl points [49]. 
SG 92 (and 96) has another fourfold crossing at the R point, which is not left invariant by fourfold rotations. Again, we use the eigenvalues of the twofold rotation $2_{001}\left(0,0, \frac{1}{2}\right)$, but this time they are \pm 1 because of the translational part. Therefore, $2_{010}\left(\frac{1}{2}, \frac{1}{2}, \frac{1}{4}\right) \mathcal{T}$ pairs different eigenvalues on the line $\mathrm{X}-\mathrm{R}$, whereas $\mathcal{T}$ pairs identical eigenvalues at $\mathrm{R}$. From lattice and low-energy models we find that the total chirality is $\mathcal{C}= \pm 2$ as for previously discussed fourfold Weyl points (see Appendix C 1).

\section{E. Fourfold quadruple Weyl points}

In the previous chapter we have shown how a fourfold double Weyl point can be made out of two symmetry-related single Weyl points on top of each other. The $\mathbf{k}$ point A in SGs 92 and 96 also has a fourfold degeneracy, but in contrast to M, it is made up from two double instead of single Weyl points. Again, they have to have the same Chern number of absolute value 2 and thus its total Chern number is $|\mathcal{C}|=4$ [75].

The irreducible representations at $\mathrm{A}$ are two and four dimensional. In principle, the eigenvalue-dependent Kramers theorem applies as introduced in Sec. III B, i.e., the combined operation $4_{001}\left(\frac{1}{2}, \frac{1}{2}, \frac{1}{4}\right) \mathcal{T}$ pairs only some of the rotation eigenstates. But the regular Kramers theorem using $\mathcal{T}$ already pairs the same $4_{001}\left(\frac{1}{2}, \frac{1}{2}, \frac{1}{4}\right)$ eigenvalues and it is always applicable. Thus, the following argument utilizes the Kramers theorem based on time-reversal symmetry alone.

Time-reversal symmetry $\mathcal{T}$ pairs the bands as $(0,2),(1,1)$, and $(3,3)$, when labeled with the parameter $p$ corresponding to the fourfold rotation eigenvalue as defined in Eq. (4.1). Analogously to the previous chapter, the combined operation $2010\left(\frac{1}{2}, \frac{1}{2}, \frac{1}{4}\right) \mathcal{T}$ pairs bands into nodal planes. Hereby, the nonsymmorphic fourfold rotation in SGs 92 and 96 modifies Eq. (4.4), where the translation on the right-hand side is now $t(1,0,-1)$ with eigenvalue 1 at $\mathrm{A}$. Together with the $k_{z}$-dependent eigenvalues the resulting pairing due to $2_{010}\left(\frac{1}{2}, \frac{1}{2}, \frac{1}{4}\right) \mathcal{T}$ is $(0,2)$ and $(1,3)$.

In conclusion, there is a twofold degeneracy $(0,2)$ as well as a fourfold degeneracy $(1,1,3,3)$, where each state in the tuple is orthogonal to the others by either different eigenvalues, i.e., different $p$, or due to Kramers theorem. We interpret the fourfold crossing as two copies of double Weyl points with $|\mathcal{C}|=2$. This chirality is determined from the symmetry eigenvalues $(1,3)$ [40]. Their chiralities must be equal because the double Weyl points are related by time-reversal symmetry.

To see this in detail, we provide a low-energy Hamiltonian of this crossing. Since most terms linear in $\mathbf{q}=\mathbf{k}-(\pi, \pi, \pi)$ vanish, terms up to quadratic order need to be considered. Up to unitary transformations, such a $4 \times 4$ Hamiltonian is restricted to a block-diagonal form with the two double Weyl Hamiltonians $H_{\mathrm{dW}}^{ \pm}$making up the blocks:

$$
\begin{aligned}
H_{\mathrm{dW}}^{ \pm}= & \left( \pm v_{x} q_{x} q_{y}+\lambda_{z} q_{z}\right) \sigma_{x}+v_{y}\left(q_{x}^{2}-q_{y}^{2}\right) \sigma_{y} \\
& +\left( \pm v_{z} q_{z}+\lambda_{x} q_{x} q_{y}\right) \sigma_{z} .
\end{aligned}
$$

See Appendix C 2 for a detailed derivation. The Chern number is equal in both blocks and the different signs only show in the dispersion when all three components of $\mathbf{q}$ are nonzero. Only then, the bands are nondegenerate. Otherwise, the eigenvalues of both Weyl points are identical, which ensures the twofold degenerate planes. Because of the splitting into nondegenerate bands and the nonzero Chern number we do not use the name Dirac point, which has been used in a prior report [75].

\section{NONSYMMORPHIC DIRAC POINTS}

Enforced Dirac points pinned to TRIMs are a common and readily accessible result of space-group symmetries. For completeness, these crossings are listed in the second column of Table II. Here we want to give another perspective by focusing on movable enforced fourfold crossings in the presence of inversion or mirror symmetries. SGs 106, 130, 133, and 138 host either movable fourfold crossings without chirality or, in the presence of inversion symmetry, movable Dirac points. The following study of their symmetry eigenvalues highlights similarities and differences. Finally, we discuss the pinned fourfold crossings enforced in SGs 108 and 142 at the point P.

\section{A. SGs 106 and 133}

The symmetry operations in SGs 106 and 133 enforce a movable fourfold crossing on the line M-A, which is part of a fourfold rotation axis. First we focus on SG 133, a centrosymmetric group, for which the movable crossing is a Dirac point. We explain its existence in terms of its symmetry eigenvalues and their connectivity within the BZ.

SG 133 contains the fourfold rotation $4_{001}\left(\frac{1}{2}, 0, \frac{1}{2}\right)$ and a glide mirror symmetry $M_{010}\left(\frac{1}{2}, 0,0\right)$. Along M-A the bands can be labeled by $p \in\{0,1,2,3\}$ referring to the eigenvalues of $4_{001}\left(\frac{1}{2}, 0, \frac{1}{2}\right)$, namely, $\exp \left[i(2 p+1) \frac{\pi}{4}\right] \exp \left(i \frac{k_{z}}{2}\right)$. An explicit calculation shows that the fourfold rotation is related by $M_{010}\left(\frac{1}{2}, 0,0\right)$ to its cube

$$
\begin{aligned}
& 4_{001}\left(\frac{1}{2}, 0, \frac{1}{2}\right) M_{010}\left(\frac{1}{2}, 0,0\right) \\
& \quad=-M_{010}\left(\frac{1}{2}, 0,0\right)\left(4_{001}\left(\frac{1}{2}, 0, \frac{1}{2}\right)\right)^{3} t(1,0,-1),
\end{aligned}
$$

where the factor of -1 stems from the spin sector. On the line M-A $\left(\pi, \pi, k_{z}\right)$ this relation pairs a band described by $p$ to the band with $p^{\prime}=3 p+1 \bmod 4$. If we denote paired bands with $p$ and $p^{\prime}$ as $\left(p, p^{\prime}\right)$, then the only two possibilities on the M-A axis are $(0,1)(2,3)$.

The second part of the argument considers the Kramers theorem at the end points of the line M-A. Time reversal forms band pairs $(0,3)(1,2)$ at $\mathrm{M}, k_{z}=0$, and $(0,1)(2,3)$ at $\mathrm{A}, k_{z}=\pi$. Finally, one obtains the degeneracy at $\mathrm{A}$ by the combined operation $\mathcal{T}^{\prime}=\mathcal{T} M_{010}\left(\frac{1}{2}, 0,0\right)$, which fulfills $\mathcal{T}^{\prime 2}=-1$ at $\mathrm{M}$ and $\mathrm{A}$. It pairs at $k_{z}=0$ the eigenvalues into the tuples $(0,2)(1,3)$ and at $k_{z}=\pi(0,0)(1,1)(2,2)(3,3)$.

We conclude that at $\mathrm{M}$ there is only one representation containing all $4_{001}\left(\frac{1}{2}, 0, \frac{1}{2}\right)$ eigenstates once: $(0,1,2,3)$, whereas at A two representations are possible, for which either $(0,0,1,1)$ or $(2,2,3,3)$ correspond to distinct sets of degenerate eigenstates. The twofold degenerate bands on the M-A line with rotation eigenstates $(0,1)$ or $(2,3)$ must interpolate between $M$ and $\mathrm{A}$. They exchange an odd number of times and thus lead to an enforced fourfold crossing as presented in Fig. 7(a). 
(a)

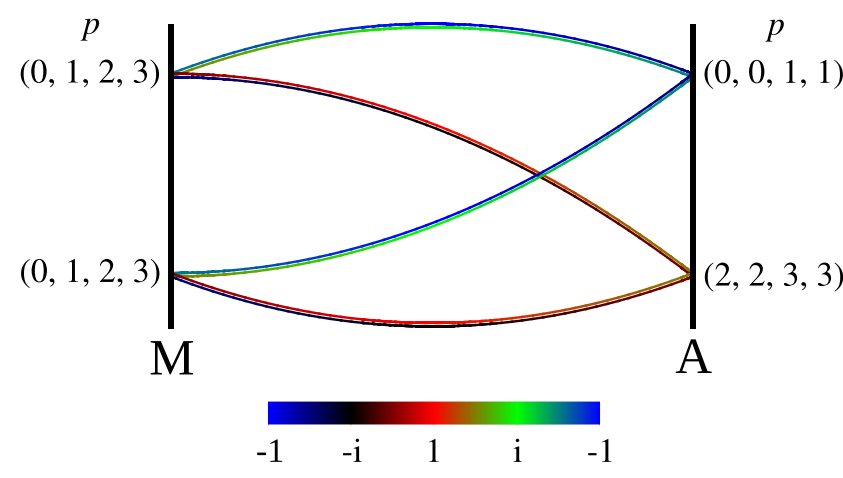

(b)

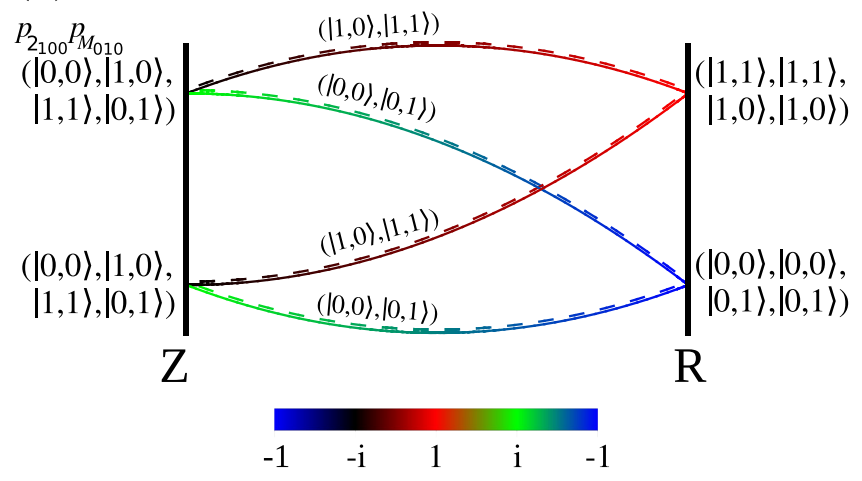

FIG. 7. Movable fourfold crossings. (a) SGs 106 and 133: the symmetry eigenvalues $\alpha_{4_{001}}$ on the M-A line are represented by the line color. For SG 133 the M-A line hosts a Dirac point, whereas for SG 106 the crossing corresponds to a fourfold point with zero chirality. (b) SGs 130 and 138: the color corresponds to the symmetry eigenvalues $\alpha_{2_{100}}$, whereas the line style (dashed) distinguishes the values of $p_{M_{010}}$. A Dirac point is enforced on the Z-R line.

So far we have not used the inversion symmetry at all. Its presence together with time reversal specifies that the bands away from M-A are twofold degenerate, thus the movable crossing is a Dirac point. Consequentially, this argument can be applied in the absence of inversion symmetry. Since SG 106 contains nearly identical fourfold rotation and mirror symmetries as SG 133, one may repeat the arguments above and find again the band structure on M-A as shown in Fig. 7(a). Yet, SG 106 is special because there is no $\mathcal{P} \mathcal{T}$ symmetry present and therefore the bands split into four nondegenerate ones away from the movable crossing. The movable crossing of SG 106 is a fourfold crossing on a mirror plane and thus has a vanishing Chern number.

The minimal number of connected bands for SGs 106 and 133 is eight (or four when excluding spin). Thereby, only the movable crossings on the M-A line are necessary to connect two sets of four bands. One concludes that SGs 106 and 133 can lead to semimetals with movable fourfold crossings at a filling of $4+8 \mathbb{N}$ electrons per unit cell. They can be thought of as two superimposed Weyl semimetals with vanishing total chirality. As such, they show two sets of surface states which can hybridize with each other unlike the Fermi arcs for a Weyl semimetal on its own. Thus, van Hove singularities appear for the surface spectrum (see Appendix B 2).

\section{B. SG 130}

In SG 130 movable Dirac points appear and they require the presence of inversion symmetry unlike the fourfold crossings of SGs 106 and 133. They appear on the line Z-R, a twofold rotation axis. A movable Dirac point cannot be understood by a single twofold symmetry alone. It would require two different ways to pair eigenvalues at the TRIMs, i.e., end points of the line and a third point along the line itself, which is impossible with only two eigenvalues.

Therefore, we must consider two symmetries at once. Indeed, SG 130 contains the off-centered screw rotation $2_{100}\left(\frac{1}{2}, 0, \frac{1}{2}\right)$ and the mirror symmetry $M_{010}\left(0, \frac{1}{2}, \frac{1}{2}\right)$. On the BZ path Z-R, e.g., $\left(k_{x}, 0, \pi\right)$, these symmetries commute and we label the bands and states by $\left|p_{2_{100}}, p_{M_{010}}\right\rangle$, where the eigenvalues $\alpha_{2_{100}}=\exp \left[i\left(2 p_{2_{100}}+1\right) \frac{\pi}{2}\right] \exp \left(i \frac{k_{x}}{2}\right)$ and $\alpha_{M_{010}}=$ $\exp \left[i\left(2 p_{M_{010}}+1\right) \frac{\pi}{2}\right] \exp \left(i \frac{k_{z}}{2}\right)$ are referred to by their respective value of $p \in\{0,1\}$. As before, round brackets are used to denote the pairing of irreducible representations denoted by the different $p$.

Inversion with time-reversal symmetry $\mathcal{P} \mathcal{T}$ pairs identical eigenvalues for off-centered symmetries on certain planes or lines [39]. On Z-R, identical $2_{100}\left(\frac{1}{2}, 0, \frac{1}{2}\right)$ eigenvalues are paired, but different eigenvalues for $M_{010}\left(0, \frac{1}{2}, \frac{1}{2}\right)$. Therefore, the pairing there is $(|0,0\rangle,|0,1\rangle)$ and $(|1,0\rangle,|1,1\rangle)$. At the TRIMs time reversal also pairs the symmetry eigenvalues. Whereas the phase of $\alpha_{2_{100}}$ depends on $k_{x}, \alpha_{M_{010}}$ is real at $Z$ and $\mathrm{R}$, resulting in the pairing $(|0,0\rangle,|1,0\rangle)$ and $(|0,1\rangle,|1,1\rangle)$ at $k_{x}=0$ and $(|0,0\rangle,|0,0\rangle),(|0,1\rangle,|0,1\rangle),(|1,0\rangle,|1,0\rangle)$, and $(|1,1\rangle,|1,1\rangle)$ at $k_{x}=\pi$. Finally, inversion $\mathcal{P}$ anticommutes (commutes) with $2_{100}\left(\frac{1}{2}, 0, \frac{1}{2}\right)$ at $\mathrm{Z}$ (at R) and anticommutes with $M_{010}\left(0, \frac{1}{2}, \frac{1}{2}\right)$ at both $\mathrm{Z}$ and $\mathrm{R}$. States related by inversion have the same energy but are not necessarily orthogonal unless at least one of their eigenvalues differs.

With this preparation we can infer all degenerate states at the TRIMs $\mathrm{Z}$ and $\mathrm{R}$. We start from $|0,0\rangle$ and apply to it $\mathcal{T}, \mathcal{P}$, or $\mathcal{P} \mathcal{T}$ and determine which eigenvalue tuple the consecutive results have. The resulting states after the application of above symmetries are at $\mathrm{Z}(|0,0\rangle,|1,0\rangle,|1,1\rangle,|0,1\rangle)$, whereas at $\mathrm{R}$ one obtains $\left(|0,0\rangle,|0,0\rangle^{\prime},|0,1\rangle,|0,1\rangle^{\prime}\right)$ and $\left(|1,1\rangle,|1,1\rangle^{\prime},|1,0\rangle,|1,0\rangle^{\prime}\right)$. Here, states orthogonal by Kramers theorem are primed for clarity. To interpolate between the fourfold degeneracies at $\mathrm{Z}$ and $\mathrm{R}$ a movable Dirac point must appear [see Fig. 7(b)].

Since the mirror symmetry $M_{001}\left(\frac{1}{2}, \frac{1}{2}, 0\right)$ is not off centered, there can be no Dirac nodal line on $k_{z}=\pi$. Therefore, the identified crossing is not part of a fourfold nodal line, which concludes our derivation of the movable Dirac points on Z-R in SG 130.

The movable Dirac point of SG 130 has been considered in [76] alongside the eightfold degeneracy at the A point appearing in SG 130 as well as 135 [77]. These double Dirac points at the A point are linear band crossings and lie at the boundary between topological insulating phases, which can be reached by breaking spatial symmetries, for example, with strain [76]. Since the eightfold point is at the boundary, one of the achievable phases must be topological but the details depend on the values of the mass terms introduced by the symmetry-breaking perturbations. 
(a)

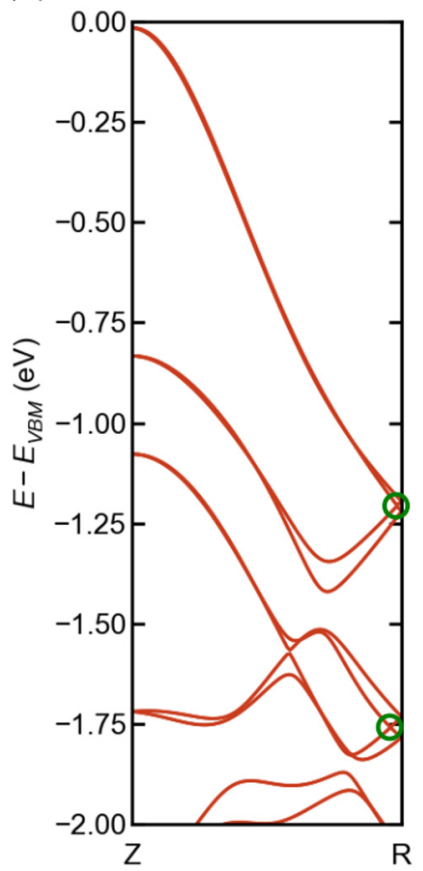

(b)

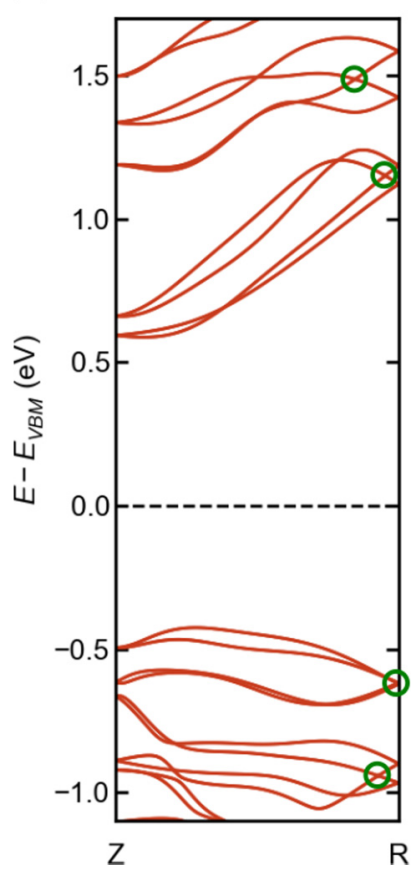

FIG. 8. Movable Dirac points in $\mathrm{AuBr}$ and $\mathrm{Tl}_{4} \mathrm{PbSe}_{3}$. Bands along the Z-R line for (a) AuBr (SG 138) and (b) $\mathrm{Tl}_{4} \mathrm{PbSe}_{3}$ (SG 130). Green circles highlight the enforced Dirac points (see Sec. V B).

\section{Material example: $\mathrm{Tl}_{4} \mathrm{PbSe}_{3}$}

$\mathrm{Tl}_{4} \mathrm{PbSe}_{3}$ [78] is an air-stable narrow-gap semiconductor which crystallizes in SG 130 (along with several other thallium tetrel chalcogenides and thallium chalcohalides), and has been studied recently for possible thermoelectric applications [79]. Its band structure is shown in Fig. 8(b) with the movable Dirac points close to the $\mathrm{R}$ point and the full band structure is given in Fig. 13(e).

\section{SG 138}

SG 138 hosts movable Dirac points on the line Z-R, which can be shown by identical arguments as in SG 130 (see Sec. V B). As the Dirac points have been discussed above, we focus here only on the distinction between both groups. Unlike SG 130, SG 138 exhibits a nontrivial topology described by a $\mathbb{Z}_{2}$ invariant, which is enforced by the connectivity of bands. Whereas the nontrivial value of the invariant is fixed for any band order, the robustness is found to be dependent on details of the system.

Since materials in SG 138 are Dirac semimetals at halffilling (gapless systems with Dirac points on the line Z-R), the invariants for topological insulators do not apply. Nevertheless, we can consider two-dimensional gapped subsystems for which the existence of surface states can be inferred from changes in the time-reversal polarization $\pi_{a}$, where we use the results and notation of [80].

To determine the time-reversal polarization $\pi_{a}$ we can employ the quantities $\delta_{\Gamma_{i}}=\prod_{m=1}^{N} \xi_{2 m}\left(\Gamma_{i}\right)$, where $\xi_{2 m}\left(\Gamma_{i}\right)$ denotes the eigenvalue of inversion $\mathcal{P}$ for the band $2 m$ at the TRIM $\Gamma_{i} \in\{\Gamma, \mathrm{X}, \mathrm{Z}, \mathrm{M}, \mathrm{R}, \mathrm{A}\}$. We consider the number of

occupied bands $N$ to be $4+8 \mathbb{N}$ for $S G 138$, i.e., the band index of the movable Dirac points. From the quantities $\delta_{\Gamma_{i}}$ one can obtain the polarization $\pi_{a}=\delta_{a 1} \delta_{a 2}$ [80]. The TRIMs labeled by $a 1$ and $a 2$ will fall on top of each other, once we terminate the system in real space. To evaluate the time-reversal polarization $\pi_{a}$ for SG 138 in a meaningful way, we consider subsystems with a band gap, e.g., the plane containing the TRIMs XMAR. Edge states of said planes appear on lines in the full surface BZ. To be more specific, the plane XMAR projects to the path $\bar{X}-\bar{M}$ in the two-dimensional surface $\mathrm{BZ}$ for a (001) termination of the full three-dimensional system and, thus, its edge states appear on this path.

In the following we give the inversion eigenvalues at the TRIMs for SG 138, which can be determined to a large extent from the commutation relations between the symmetries. Due to time-reversal symmetry $\mathcal{T}$ each inversion eigenvalue always appears twice, connected as a Kramers pair. This is already considered by taking only even band indices $2 m$ for $\xi_{2 m}\left(\Gamma_{i}\right)$ in the expression for $\delta_{\Gamma_{i}}$ [80]. Furthermore, for SG 138 all TRIMs except $\Gamma$ are fourfold degenerate. The nonsymmorphic mirror symmetries relate opposite inversion eigenvalues at the points $\mathrm{X}, \mathrm{R}, \mathrm{M}$, and $\mathrm{Z}$. At $\mathrm{A}$, an eigenstate can be labeled by inversion and mirror eigenvalues simultaneously. The application of $\mathcal{T}$ and the twofold screw rotation yields that four bands with the same inversion eigenvalue are degenerate at $\mathrm{A}$. We can thus explicitly give the values of $\delta_{\Gamma_{i}}$ for the TRIMs $\delta_{\mathrm{X}}=\delta_{\mathrm{R}}=\delta_{\mathrm{M}}=\delta_{\mathrm{Z}}=\xi_{2}(\mathrm{X}) \xi_{4}(\mathrm{X})=-1$ and $\delta_{\mathrm{A}}=\xi_{2}(\mathrm{~A}) \xi_{4}(\mathrm{~A})=+1$, whereas $\delta_{\Gamma}= \pm 1$ is not determined from symmetry alone. Note that $\delta_{\mathrm{A}}=+1$ is independent of the band order, i.e., the value of $\xi_{2}(\mathrm{~A})=\xi_{4}(\mathrm{~A})= \pm 1$.

Below we discuss the surface states for a slab with (001) termination, which we compare to the explicit calculation for a generic model (see Appendix B 3). For the plane XMAR there are two time-reversal polarizations $\pi_{1}=\delta_{\mathrm{X}} \delta_{\mathrm{R}}=1$ and $\pi_{2}=\delta_{\mathrm{M}} \delta_{\mathrm{A}}=-1$. This difference between $\pi_{1}$ and $\pi_{2}$ leads to surface states on the line $\overline{\mathrm{X}}-\overline{\mathrm{M}}$, which is confirmed by the surface spectrum [see Fig. 16(c)]. The number of surface states depends on the details of the system, but by the above argument SG 138 ensures that the surface states cross the gap connecting valence and conduction bands an odd number of times. Computing the product $\pi_{1} \pi_{2}=(-1)^{\nu_{1}}$ analogously to the weak invariant [80] yields $v_{1}=1$.

To support this approach we compare SG 138 to SG 130. Although the mirror operations of SGs 130 and 138 are identical and the enforced inversion eigenvalues are in principle the same, no topological Dirac surface state appears for SG 130 on the line $\overline{\mathrm{X}}-\overline{\mathrm{M}}$. The difference between SGs 130 and 138 is the band touching at the point A. Due to this eightfold crossing in SG 130 the plane XMAR is gapless and the same number of positive and negative inversion eigenvalues are present at A. Also note that the movable Dirac points, which appear for both space groups, are trivial because $(\mathcal{P} \mathcal{T})^{2}=-1[81,82]$. Both remarks support the interpretation that the time-reversal polarizations $\pi_{a}$ capture the topology (see the further analysis in Appendix B 3).

A robust topological phase does not lose its surface states or topological invariant for any perturbation that preserves the bulk band gap and the protecting symmetry. This distinguishes strong from weak topological insulators [80]. To apply this classification to SG 138 we consider arbitrary small 
perturbations that break the symmetries except inversion. Thus, the order of bands at the TRIMs does not change and all $\delta_{\Gamma_{i}}$ will be preserved. Once the Dirac points on the lines $\mathrm{Z}$-R are slightly gapped, we can calculate the strong invariant $v$ by $(-1)^{v}=\prod_{\Gamma_{i}} \delta_{\Gamma_{i}}=\delta_{\Gamma}$. Hence, the band order at $\Gamma$ by itself determines the stability of the topological phase.

To summarize the results for SG 138, we find aside from four movable Dirac points that the spatial symmetries enforce a nontrivial weak topological invariant. We find that the overall robustness of the topological phase depends on the order of bands at the point $\Gamma$.

\section{Material example: $\mathrm{AuBr}$}

The primitive tetragonal polymorph of $\mathrm{AuBr}$, crystallizing in SG 138 [83], is an example of a material with Dirac points along the Z-R line, as discussed in Sec. V. It consists of layers of polymeric $\mathrm{Au}-\mathrm{Br}$ zigzag chains, and can be grown as yellow-brown crystals by vapor transport. In Fig. 8(a) we show the first-principles band dispersions of $\mathrm{AuBr}$ along the $\mathrm{Z}-\mathrm{R}$ direction. All bands are twofold Kramers degenerate due to time-reversal and inversion symmetry. Along Z-R we observe groups of four connected bands that form an odd number of Dirac crossings. AuBr is insulating with a band gap of about $2 \mathrm{eV}$ [see Fig. 13(d)]. This large gap makes it difficult to experimentally measure the band structure using photoemission or scanning tunneling probes.

\section{SG 108}

SG 108 contains a fourfold crossing that is not pinned to a TRIM but to the point P [84]. Due to the presence of mirror symmetries, it does not carry a chirality as topological charge. Yet, since a fourfold crossing away from TRIMs is a unique feature of some tetragonal space groups, we will discuss its origin.

The fourfold crossing in SG 108 can be understood with the eigenvalues $\exp \left[i\left(1+2 p_{2_{001}}\right) \frac{\pi}{2}\right]$ of $2_{001}(0,0,0)$. At $\mathrm{P}$ the Kramers theorem is applicable to the combined symmetry $\mathcal{T} M_{010}\left(0,0, \frac{1}{2}\right)$, which pairs identical $p_{2_{001}}$ values $(0,0)$ and $(1,1)$. Furthermore, the point $\mathrm{P}$ is left invariant by $M_{1 \overline{1} 0}\left(0,0, \frac{1}{2}\right)$, which relates different $p_{2_{001}}$ yielding the pair $(0,1)$. Therefore, four bands are degenerate at $\mathrm{P}$. The fourfold crossing splits into nondegenerate bands away from $\mathrm{P}$ except along the lines P-X and P-N.

\section{E. SG 142}

SG 142 is the only tetragonal SG with a Dirac crossing at the point P. A different argument as for SG 108, which contains a fourfold crossing at $\mathrm{P}$, must be used because its corresponding twofold rotation $2_{001}\left(\frac{1}{2}, 0, \frac{1}{2}\right)$ is off centered, time reversal with mirror or inversion symmetries pair already identical eigenvalues.

SG 142 is similar to SG 110 (cf. Sec. VIC for more details) because also in SG 142 three nonsymmorphic operations $2_{001}\left(\frac{1}{2}, 0, \frac{1}{2}\right), M_{110}\left(\frac{3}{4}, \frac{1}{4}, \frac{1}{4}\right)$, and $M_{1 \overline{1} 0}\left(\frac{3}{4}, \frac{3}{4}, \frac{3}{4}\right)$ commute on the line X-P. The product of the corresponding eigenvalues must fulfill $\alpha_{2_{001}} \alpha_{M_{110}} \alpha_{M_{110}}=\exp \left(i 3 k_{z} / 2\right)$, leading to four possible eigenvalue configurations $|0,0,1\rangle,|0,1,0\rangle,|1,0,0\rangle,|1,1,1\rangle$ along the X-P line, where the parameters $p$ defined analogously to before label the states $\left|p_{2_{001}}, p_{M_{110}}, p_{M_{110} 0}\right\rangle$. Time reversal with inversion $\mathcal{P} \mathcal{T}$ pairs them in groups of two: $(|0,0,1\rangle,|0,1,0\rangle)$ and $(|1,0,0\rangle,|1,1,1\rangle)$.

There are irreducible representations of dimensions 2 and 4 at the $\mathrm{P}$ point, due to an eigenvalue-dependent Kramers theorem (see Sec. III B). The combination $\mathcal{T} 4_{001}\left(\frac{1}{4}, \frac{3}{4}, \frac{1}{4}\right)$ leads to orthogonal new states at $\mathrm{P}$, if applied to a state with $p_{2_{001}}=1$. In this process the values of $p_{2_{001}}$ remain unchanged and the twofold degeneracy $(|1,0,0\rangle,|1,1,1\rangle)$ is doubled and results in the Dirac point at $\mathrm{P}$.

\section{TWOFOLD WEYL LINES}

Nodal lines pinned to rotation axes or equivalently the intersection of two mirror planes are a common feature. These pinned degeneracies can be understood from the structure of the little group and are tabulated $[63,64]$. The identification of movable nodal lines, on the other hand, poses more intricacies, which we discuss in the following sections. Here, we begin by considering the concept of glide mirror symmetries in the presence of time reversal, before we move to more complex nodal lines. In the simplest case, so-called hourglass nodal lines are found, which were classified on the grounds of compatibility relations [85]. Our approach uses the explicit symmetry eigenvalues instead of representations and facilitates the derivation of other movable nodal lines between TRIMs (Sec. VI A).

In the absence of inversion, any glide mirror symmetry naturally leads to twofold nodal lines. Applying a glide mirror symmetry with translation $t_{\perp}$ within the mirror plane twice results in a lattice translation by $2 t_{\perp}$. Consequently, the eigenvalues need to be the square roots of this lattice translation and are $k$ dependent,

$$
\alpha_{p}(\mathbf{k})=\exp \left[i(2 p+1) \frac{\pi}{2}\right] \exp \left(i \mathbf{k} \cdot t_{\perp}\right),
$$

where $t_{\perp}$ is the projection of the translational part to the mirror plane and we use $p \in\{0,1\}$ as label. When traversing the BZ, these eigenvalues exchange at least once.

In the presence of other symmetries, degeneracies between states with different eigenvalues or, through Kramers theorem, identical eigenvalues can be enforced. For example, at TRIMs in the $\mathrm{BZ}$ where $\mathbf{k} \cdot t_{\perp}=0 \bmod 2 \pi$ the values of $p$ are paired as $(0,1)$, while for $\mathbf{k} \cdot t_{\perp}=\pi \bmod 2 \pi$ they are paired to be $(0,0),(1,1)$. On any path within the mirror plane connecting two such points with different eigenvalue pairing, the standard argument for hourglass dispersions can be invoked and thus each such path must contain an odd number of band crossings. These enforced crossings form movable Weyl nodal lines, away from high-symmetry points.

We specify movable nodal lines in column 3 of Table I by grouping points according to their eigenvalue pairing. The left-hand side of a tuple always contains points with pairs of alternating signs, whereas identical eigenvalues are paired at all points on the right-hand side. The nodal line then has to be in-between these sets and in the plane containing all points of the tuple. For example, in SG 100 we denote with $(\Gamma-Z$; $\mathrm{X}, \mathrm{R})(4)$ that at any point on the line $\Gamma-\mathrm{Z}$ the value of $p$ is paired as $(0,1)$, whereas at $X$ and $R$ the pairs $(0,0)$ and $(1,1)$ are 
formed. Hence, four bands are connected on any path between $\Gamma-\mathrm{Z}$ to $\mathrm{X}$ or $\mathrm{R}$, each forming an hourglass structure. Such a path exhibits a band connectivity as depicted in Fig. 4(a) for the movable Weyl points on a high-symmetry line. Within the tetragonal space groups, fourfold crossings do appear in planes with movable nodal lines. It turns out that on a path containing such a fourfold crossing, no additional crossing is enforced. Applied to SG 102 with the nodal line denoted by $(\Gamma-Z-R ; X)(4)$ this means that a path from $Z$ to $X$ does not necessarily cross a nodal line. We note this by writing a cursive $Z$ instead of $Z$ on the line of identical paired eigenvalues $\Gamma-Z-R$. One can confirm from the irreducible representations that there are no fourfold crossings with only one value of the mirror eigenvalue in the tetragonal SGs [63].

In conclusion, we find that SGs 100, 102, 104, 106, 108, $109,110,117,118,120$, and 122 enforce on at least one mirror plane a movable twofold nodal line. Using the previous discussion, it can be understood that intersecting mirror planes may give rise to nodal chain metals [10]. We will briefly discuss the relevance to tetragonal space groups and give a material example. Then, we focus on SG 110, although it does not contain a nodal chain extended in reciprocal space, it exhibits intersecting nodal lines and a band connectivity allowing in principle for a simple Fermi surface. But before we move to these complex configurations of mirror planes, we discuss a type of nodal lines which is easily missed despite its conceptual simplicity. These nodal lines are also movable, except that they are pinned to certain TRIMs.

\section{A. Almost movable nodal lines}

In this section we will discuss twofold almost movable nodal lines, which are enforced features pinned at a highsymmetry point but which may be moved freely everywhere else in the mirror plane. Before discussing the affected $\mathrm{SG}$, we derive the existence of this type of nodal line in body-centered SGs.

To begin, suppose there is a single (glide) mirror symmetry, with eigenvalues $\pm i$ at a TRIM on the mirror plane. Time reversal pairs the symmetry eigenvalues $+i$ and $-i$ yielding twofold degenerate bands at this TRIM. Let $\mathbf{q}$ be the coordinate of a point in the mirror plane relative to the TRIM. Time-reversal symmetry relates $\mathbf{q}$ to $-\mathbf{q}$, while exchanging the mirror eigenvalues of opposite phase, + with - . If one chooses a path leading from $\mathbf{q}$ to $-\mathbf{q}$ within the mirror plane and without crossing the TRIM, we know that the mirror eigenvalues must change. As the eigenvalues may only change if bands cross, we can conclude from the generality of the path that the twofold degeneracy at the TRIM is part of a nodal line. We refer to these nodal lines as almost movable nodal lines because they are unpinned everywhere except for a finite set of points, i.e., the TRIMs which enforce them.

Almost movable nodal lines are unlike pinned and movable nodal lines. Although the above argument holds for all TRIMs in mirror planes with eigenvalue pairing of opposite signs, i.e., $p=0$ and 1 in Eq. (6.1), often there are further symmetries present which pin the nodal line to a rotation axis. Pinned nodal lines arrange for the exchange of eigenvalues in the same way, but they are completely fixed to a straight line by the anticommutation of symmetries or by Kramers theorem
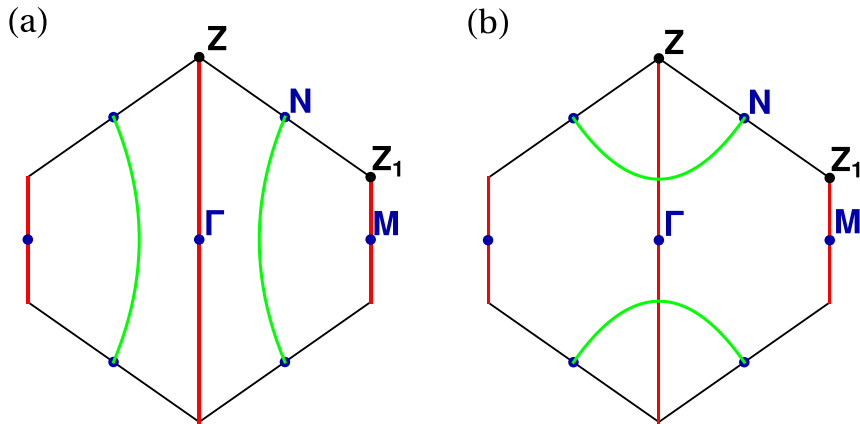

FIG. 9. Almost movable nodal lines (green) and pinned nodal lines (red) in mirror planes of SGs 107, 109, and 119. The connectivity of almost movable nodal lines is not determined by symmetry alone. (a) Nodal line connecting distinct $\mathrm{N}$ points. (b) Same as (a), but intersecting the pinned nodal line.

applied to the combination of a glide mirror symmetry with time reversal. Their appearance can therefore be understood from the group structure of the involved symmetries alone and they can be found in tabulated irreducible representations of the corresponding little groups [63]. The other extreme is movable nodal lines, which are only constrained in the sense that they mediate an exchange of mirror eigenvalues within the mirror plane, as we have discussed at the beginning of Sec. VI. This differs from the almost movable nodal lines in that the latter are not responsible for exchanging symmetry eigenvalues between different TRIMs. Studying compatibility relations along high-symmetry paths would not explain almost movable nodal lines $[63,85]$. Thus, they are not just movable nodal lines pinned at a finite number of points in the BZ, but they exist because nodal lines have no end points.

A SG with almost movable lines needs to have a TRIM in a mirror plane that is not part of a rotation axis, i.e., the little group of the TRIM contains only the reflection. In the tetragonal crystal system, this is only possible at $\mathrm{N}$ in bodycentered space groups with crystallographic point group $4 \mathrm{~mm}$ or $\overline{4} 2 m$. Of those, only SGs 107,109 , and 119 pair $\pm i$ at $\mathrm{N}$. For these SGs we illustrate the possible connectivity of almost movable lines qualitatively in Fig. 9. Finally, there is a fourth case in the tetragonal SGs of an almost movable line found at $\mathrm{P}$ in $\mathrm{SG} 110$. $\mathrm{P}$ is not invariant under time reversal alone, but under the antiunitary symmetry $M_{010}\left(0,0, \frac{1}{2}\right) \mathcal{T}$. It creates a pair of eigenvalues with opposite signs $(0,1)$ for $p$ in the definition of the 110-mirror eigenvalues [cf. Eq. (6.1)], and creates an almost movable nodal line in the corresponding invariant plane. A detailed discussion and derivation can be found in Appendix C 3.

The almost movable lines are indicated in the same column as the movable lines, i.e., the third column in Table I. We use the same notation as for movable nodal lines, e.g., for SG 107 we write $\left(\mathrm{N}, \Gamma-\mathrm{Z}, \mathrm{M}-\mathrm{Z}_{1} ;-\right)(2)$. This expression is understood as before. Bands at the points and lines $\mathrm{N}, \Gamma-\mathrm{Z}$, and $\mathrm{M}-\mathrm{Z}_{1}$ are twofold degenerate and comprise both mirror eigenvalues. But here the second entry, where we would denote points in the mirror plane of identical pairing remains empty "-". Note that $\Gamma-Z$ and $M-Z_{1}$ exhibit pinned nodal lines, whereas the point $\mathrm{N}$ is crossed by an almost movable nodal line. The nodal line passing through $\mathrm{N}$ may cross but cannot end at the pinned 
(a)

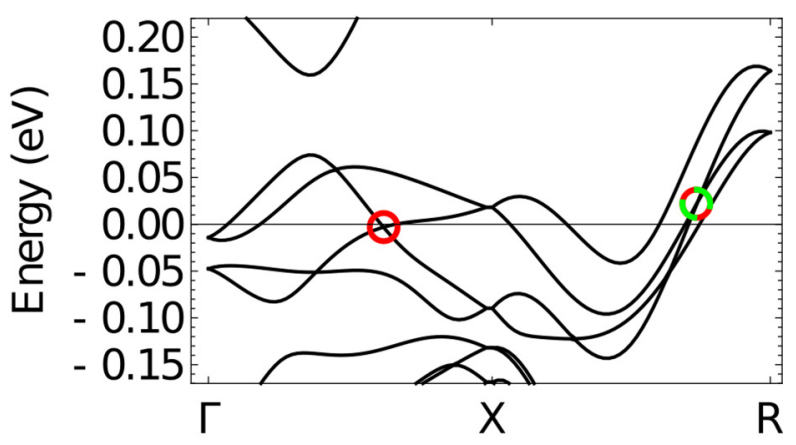

(b)

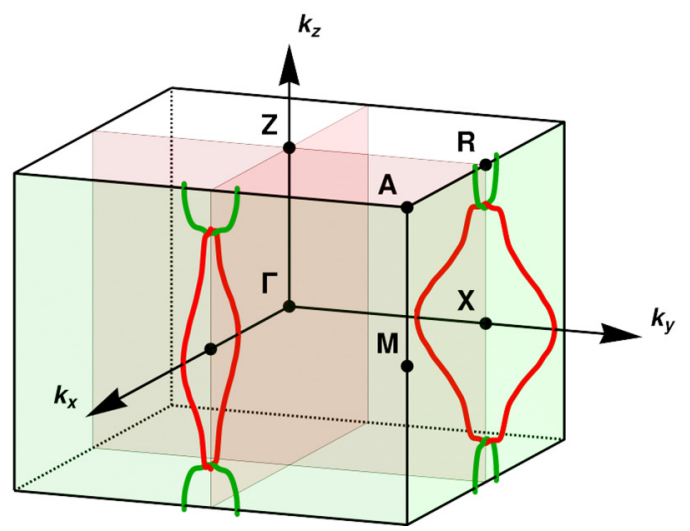

FIG. 10. Nodal chain metal $\mathrm{Ba}_{5} \mathrm{In}_{4} \mathrm{Bi}_{5}$ in SG 104. (a) Electron bands close to the Fermi energy exhibiting nodal lines. Circles mark points on the nodal chain and their color refers to the color code in (b). On the line X-R the red and green sections of the nodal chain cross. (b) Nodal chain formed by the bands in (a) represented as lines in the Brillouin zone. Red: nodal lines in a mirror plane defined by $k_{x}=0$ or $k_{y}=0$. Green: nodal lines in a mirror plane defined by $k_{x}=\pi$ or $k_{y}=\pi$

lines like $\Gamma-Z$ (cf. Appendix B 1 with SG 119 as an example). SG 110 is special in that its almost movable nodal lines do not pass a TRIM but rather the point $\mathrm{P}$, and they do not exist for all bands (see Sec. VIC).

\section{B. Nodal chain metals}

Nodal chain materials are characterized by enforced, movable nodal lines that touch on the intersections of their mirror planes [10]. Several tetragonal SGs, i.e., 102, 104, 109, 118, and 122 , fall into this category. Nodal chain metals appear, when there are two intersecting mirror planes, whose eigenvalues are each paired differently for the two high-symmetry points on the intersecting line. A detailed discussion can be found in [10]. Note that systems with nodal chains always have other Fermi surfaces aside from the nodal chain.

\section{Material example: $\mathrm{Ba}_{5} \mathrm{In}_{4} \mathrm{Bi}_{5}$}

$\mathrm{Ba}_{5} \mathrm{In}_{4} \mathrm{Bi}_{5}$, which crystallizes in SG 104 , is an electrondeficient polar intermetallic formed by the reduction of In and $\mathrm{Bi}$ with $\mathrm{Ba}$ [86]. Its DFT band structure is presented in Figs. 10 and 13(c). The nodal chain of $\mathrm{Ba}_{5} \mathrm{In}_{4} \mathrm{Bi}_{5}$ is in close proximity to the Fermi energy presenting an experimentally accessible platform for the study of nodal chains [see (a)

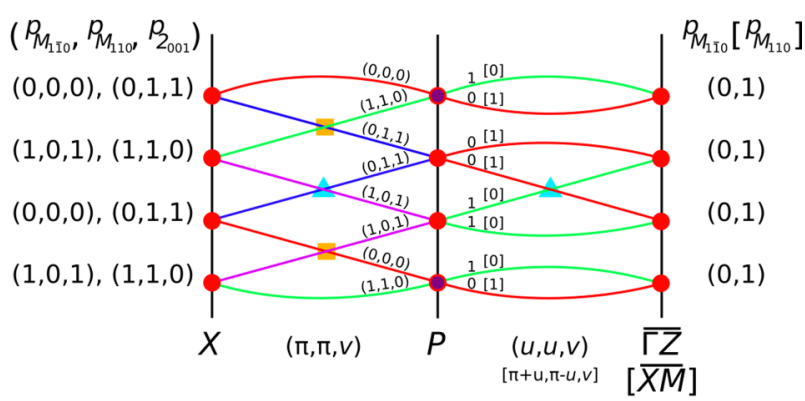

(b)

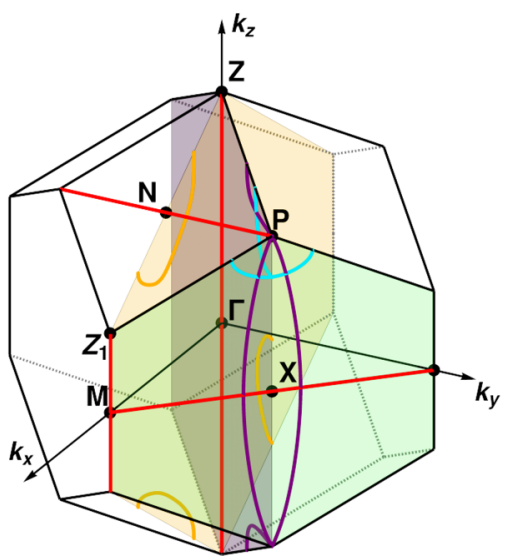

FIG. 11. Band crossings in SG 110 including the nodal armillary sphere. (a) Band connectivity, sets of symmetry eigenvalues, and enforced crossings on the line $(\pi, \pi, v)$ and on the planes $(u, u, v)$ and $(\pi+u, \pi-u, v)$, which includes $\Gamma-\mathrm{Z}$ and $\mathrm{X}-\mathrm{M}$, respectively. Eigenvalues for the plane $(\pi+u, \pi-u, v)$ are given in square brackets. Turquoise triangles: nodal armillary sphere. Yellow squares: movable nodal lines forming an hourglass structure on a single mirror plane. Red circles: pinned nodal lines, which can be overlapped by purple circles indicating additional almost movable nodal lines. (b) Qualitative arrangement of enforced nodal lines in the 3D Brillouin zone with the same color code as the crossings in (a). Additional copies due to fourfold rotation are omitted.

Fig. 10(a)]. We show the shape of the nodal chain within reciprocal space for the bands close to the Fermi energy in Fig. 10(b).

\section{Intersecting nodal lines of SG 110}

We now want to discuss the special case of the bodycentered SG $110\left(I 4_{1} c d\right)$ [10]. It combines all types of nodal lines we have encountered so far, movable, almost movable, and pinned nodal lines. We focus on the (almost) movable nodal lines, which intersect without forming a nodal chain. The qualitative results are summarized in Fig. 11.

The movable nodal lines on mirror planes appear between high-symmetry points and pinned nodal lines. To enforce the pinned nodal lines, the twofold rotation $2_{001}\left(\frac{1}{2}, \frac{1}{2}, \frac{1}{2}\right)$ may relate eigenvalues of mirror symmetries, e.g., $M_{010}\left(0,0, \frac{1}{2}\right)$. As defined in Eq. (6.1), $p_{M_{010}}$ labels the eigenvalue of $M_{010}\left(0,0, \frac{1}{2}\right)$, i.e., $\exp \left[i\left(2 p_{M_{010}}+1\right) \frac{\pi}{2}\right] \exp \left(i \frac{k_{z}}{2}\right)$. Both symmetries commute on X-P and anticommute on $\Gamma-Z-M$. Thus, only $\Gamma-\mathrm{Z}-\mathrm{M}$ is twofold degenerate and $p_{M_{010}}$ values are paired $(0,1)$. 
Another twofold degenerate line with $(0,1)$ pairing results from Kramers theorem applied to the combined operation $\mathcal{T} M_{110}\left(0, \frac{1}{2}, \frac{3}{4}\right)$ on the line X-M, which can be parametrized by $\mathbf{k}=(\pi+u, \pi-u, 0)$ with $u \in[-\pi, \pi]$. Kramers theorem applies because the condition $\left(\mathcal{T} M_{110}\left(0, \frac{1}{2}, \frac{3}{4}\right)\right)^{2}=$ $\exp (-i u) \neq 1$ holds almost everywhere (see Sec. III B 2). The third pinned nodal line is enforced by $M_{010}\left(0,0, \frac{1}{2}\right) \mathcal{T}$ on N-P, where $\left(M_{010}\left(0,0, \frac{1}{2}\right) \mathcal{T}\right)^{2}=-1$. All pinned nodal lines are shown as red lines in Fig. 11.

We can readily assess the plane $\Gamma \mathrm{NM}$ because it is bounded by the twofold degenerate rotation axis $\Gamma-Z-M$. Therefore, $M_{010}\left(0,0, \frac{1}{2}\right)$ and time-reversal symmetry lead to an hourglass structure between any point on $\Gamma$-Z-M with $(0,1)$ pairing and the TRIM $\mathrm{N}$ which exhibits the eigenvalue pairs $(0,0)$ and $(1,1)$. The resulting movable nodal lines in the $\Gamma \mathrm{NM}$ plane connect four bands and are denoted in Table I as $\left(\Gamma-\mathrm{Z}, M-\mathrm{Z}_{1} ; \mathrm{N}\right)(4)$.

A more intricate argument is needed to understand the enforced band structure on the remaining two distinct planes because they share the rotation axis X-P with nontrivial pairing. On the line X-P three symmetries commute: $M_{1 \overline{1} 0}\left(\frac{1}{2}, 0, \frac{1}{4}\right), M_{110}\left(0, \frac{1}{2}, \frac{3}{4}\right)$, and $2_{001}\left(\frac{1}{2}, \frac{1}{2}, \frac{1}{2}\right)$. Their eigenvalues on the line X-P, $\left(\pi, \pi, k_{z}\right)$ are

$$
\begin{aligned}
& \alpha_{M_{1 \overline{1} 0}} \stackrel{\text { X-P }}{=} \exp \left[i\left(2 p_{M_{1 \overline{1}}}+2\right) \pi / 2\right] \exp \left(i k_{z} / 4\right), \\
& \alpha_{M_{110}} \stackrel{\text { X-P }}{=} \exp \left[i\left(2 p_{M_{110}}+1\right) \pi / 2\right] \exp \left(i 3 k_{z} / 4\right), \\
& \alpha_{2_{001}}=\exp \left[i\left(2 p_{2_{001}}+1\right) \pi / 2\right] \exp \left(i k_{z} / 2\right) .
\end{aligned}
$$

Each band shall be labeled as $\left|p_{M_{110}}, p_{M_{110}}, p_{2_{001}}\right\rangle$, where each $p \in\{0,1\}$.

Before we can relate the states on X-P we have to determine the possible combinations $\left(p_{M_{1 \overline{10}}}, p_{M_{110}}, p_{2001}\right)$. The states are restricted because the product of all symmetries is a translation $M_{1 \overline{1} 0}\left(\frac{1}{2}, 0, \frac{1}{4}\right) M_{110}\left(0, \frac{1}{2}, \frac{3}{4}\right) 2_{001}\left(\frac{1}{2}, \frac{1}{2}, \frac{1}{2}\right)=$ $t\left(\frac{1}{2},-\frac{1}{2}, \frac{3}{2}\right)$. As the bands are generally nondegenerate along $\mathrm{X}-\mathrm{P}$, the product of eigenvalues must fulfill $\alpha_{M_{110}} \alpha_{M_{110}} \alpha_{2_{001}}=$ $\exp \left(i \frac{3 k_{z}}{2}\right)$ [37]. One concludes that $p_{M_{1 \overline{1} 0}}+p_{M_{110}}+p_{2001}$ $\bmod 2=0$ and thereby the only possible bands on X-P are $|0,0,0\rangle,|1,1,0\rangle,|1,0,1\rangle$, and $|0,1,1\rangle$.

On the line X-P no further symmetries apply, but timereversal symmetry $\mathcal{T}$ at $\mathrm{X}$ and $\mathcal{T} M_{010}\left(0,0, \frac{1}{2}\right)$ at $\mathrm{P}$ each invoke Kramers theorem. At $X$ time reversal yields two different twofold bands $(|0,0,0\rangle,|0,1,1\rangle)$ and $(|1,1,0\rangle,|1,0,1\rangle)$. Notice that the pairing of $M_{110}\left(0, \frac{1}{2}, \frac{3}{4}\right)$ eigenvalues at $\mathrm{X}$, i.e., $(0,1)$ is consistent with the action of $\mathcal{T} M_{110}\left(0, \frac{1}{2}, \frac{3}{4}\right)$ on the line X-M discussed before. For $\mathrm{P}$ we have to consider how the three eigenvalues in Eqs. (6.2)-(6.4) are related by $\mathcal{T} M_{010}\left(0,0, \frac{1}{2}\right)$. When acting on a generic state on the right, one obtains the following relations:

$$
\begin{aligned}
& M_{110}\left(0, \frac{1}{2}, \frac{3}{4}\right) T M_{010}\left(0,0, \frac{1}{2}\right) \\
& \quad=\exp \left[i \pi\left(-2 p_{M_{110}}-1\right) / 2\right] \exp \left(i 3 k_{z} / 4\right) T M_{010}\left(0,0, \frac{1}{2}\right), \\
& 2_{001}\left(\frac{1}{2}, \frac{1}{2}, \frac{1}{2}\right) T M_{010}\left(0,0, \frac{1}{2}\right) \\
& \quad=\exp \left[i \pi\left(-2 p_{2_{001}}-3\right) / 2\right] \exp \left(i k_{z} / 2\right) T M_{010}\left(0,0, \frac{1}{2}\right) .
\end{aligned}
$$

Thus, at $\mathrm{P}$ the band doublets $(|0,0,0\rangle,|1,1,0\rangle)$, $(|1,0,1\rangle,|1,0,1\rangle)$, and $(|0,1,1\rangle,|0,1,1\rangle)$ are independent and degenerate. If the representations are ordered in energy such that the minimal number of crossings appear, then nodal lines on different mirror planes cross on the X-P axis, as shown by the turquoise lines in the visualization of SG 110 in Fig. 11(b). Unlike for nodal chain metals, the nodal lines do not need to extend over the full height of the Brillouin zone. To our knowledge, a similar arrangement of bands has only been discussed in systems without spin-orbit coupling. For the cubic SG 221, three intersecting planes with joined nodal lines were considered $[87,88]$, whereas in the orthorhombic SG 61 two intersecting nodal lines were referred to as a nodal armillary sphere [89]. These nodal lines gap out once spin-orbit coupling is relevant, unlike the feature of SG 110 presented here. There are no enforced crossings at the same energy as the connected nodal lines around P. If no accidental crossings or band pockets occur and the filling is $8 n+4$ $\left(n \in \mathbb{N}_{0}\right)$ electrons per unit cell, materials in SG 110 with a weak dispersing nodal line are not just metals with nodal points, but genuine enforced semimetals.

At different band number we find additional nodal lines appearing in the same mirror plane around different highsymmetry points. We denote this by labeling the nodal lines as $(\Gamma-Z, P ; X)(4)$ and $(\Gamma-Z, X ; P)(4)$ in Table I and show them as orange lines in Fig. 11(b). Therefore, the combination of different connectivities raises the number of connected bands to $8 \mathbb{N}$.

Furthermore, SG 110 exhibits almost movable nodal lines as introduced in Sec. VIA. They can be inferred from the pairing of symmetry eigenvalues at the point $\mathrm{P}$ we already derived. Out of the possible representations only the paired states $(|0,0,0\rangle,|1,1,0\rangle)$ contain different mirror eigenvalues. Hence, only twofold degeneracies of this type are part of almost movable nodal lines. A possible arrangement of these nodal lines is displayed by purple lines in Fig. 11(b), where the relevant crossings at $\mathrm{P}$ are highlighted in Fig. 11(a) by a purple dot. These almost movable nodal lines may intersect the pinned nodal lines shown in red, whereas they are at a different band index, i.e., different energy, than the turquoise colored nodal armillary sphere and thus do not cross them in the simplest case. See Appendix C 3 for a detailed analysis of bands in the vicinity of the point $\mathrm{P}$.

\section{FOURFOLD WEYL NODAL LINES}

Fourfold degenerate Weyl nodal lines are symmetryenforced degeneracies which split into nondegenerate bands when moving away from the nodal line in almost all directions. This distinguishes them from Dirac lines, which are also fourfold degenerate, but split into twofold degenerate states in all perpendicular directions. Fourfold degenerate nodal lines are enforced in band structures of SGs 113 and 114. The generators of these two SGs are translations, a fourfold rotoinversion $\overline{4}_{001} \equiv 4_{001}(0,0,0) \mathcal{P}$ and a twofold screw rotation $2_{010}\left(\frac{1}{2}, \frac{1}{2}, c\right)$ around an axis perpendicular to it, where the factor $c=0, \frac{1}{2}$ distinguishes between SG 113 and SG 114 and is not relevant in the following discussion.

The fourfold degenerate line follows from the two antiunitary symmetries created by combining the generators with 
time-reversal symmetry, $\overline{4}_{001} \mathcal{T}$ and $2_{010}\left(\frac{1}{2}, \frac{1}{2}, c\right) \mathcal{T}$. As mentioned before, $2_{010}\left(\frac{1}{2}, \frac{1}{2}, c\right) \mathcal{T}$ leaves points in the two planes $k_{y}=0$ and $\pi$ invariant and squares to -1 on the latter plane, therefore enforcing twofold degenerate nodal planes. $\overline{4}_{001} \mathcal{T}$ squares to $-2_{001}$ and leaves the lines $\Gamma-\mathrm{Z}$ and $\mathrm{M}-\mathrm{A}$ invariant. It further fulfills the conditions of Kramers theorem (3.1) on these lines since the eigenvalues of $2_{001}$ are $\pm i$.

On the M-A axis, pairing conditions of both symmetries are present, resulting in a pinned fourfold degenerate line. To track the pairing from each of these two antiunitary symmetries, we label the states with the sign of their $2_{001}$ eigenvalues $\pm i$. Since $2_{001}$ and $\overline{4}_{001} \mathcal{T}$ naturally commute, the eigenvalues are related only via complex conjugation, i.e., the pairing is $(+,-) .2_{010}\left(\frac{1}{2}, \frac{1}{2}, c\right) \mathcal{T}$, on the other hand, anticommutes with $2_{001}$, adding another sign change to the conjugated eigenvalue. Hence, it pairs $(+,+)$ and $(-,-)$. In combination, we find the fourfold degeneracy of the nodal line with the pairing $(+,+,-,-)$. This feature has recently been reported as a Dirac line [90], however, apart from the planes $k_{y}=\pi$ and $k_{x}=\pi$, the bands are nondegenerate. The dispersion perpendicular to the nodal line is linear and remains twofold degenerate in the nodal planes $k_{x}=\pi$ and $k_{y}=\pi$. For constant $k_{z}$, this is the same structure seen in the fourfold Weyl point shown in Fig. 5(b).

The topological protection of this feature can best be understood from the linearized Hamiltonian around the nodal line (see Appendix C 4). It can be transformed into blockdiagonal form via a $\mathbf{k}$-independent, unitary matrix. Each of the blocks describe one independent Weyl line, each protected by a $\pi$ Berry phase.

\section{Material example: $\mathrm{NaSn}_{5}$}

$\mathrm{NaSn}_{5}$ crystallizes in SG 113 [91] and shows the fourfold Weyl line along M-A and the twofold degeneracy at the BZ boundary, which can be seen as fourfold and twofold degeneracies along high-symmetry lines in Fig. 13(g). It is metallic and the fourfold nodal line crosses the Fermi energy. A closeup of bands calculated from first principles in the plane parametrized by $\mathbf{k}=\left(\pi+\delta, \pi+\delta, k_{z}\right)$ is shown in Fig. 12. It shows the dispersion of the nodal line along $k_{z}$ as well as the separation into individual bands for nonvanishing $\delta$, with a linear dispersion only for very small values. The same features have been reported in band-structure calculations for $\mathrm{Pd}_{4} \mathrm{~S}$, which crystallizes in SG 114 [90].

\section{NONSYMMORPHIC NODAL PLANES}

Band degeneracies may not only appear as points and lines but are known to occur also as two-dimensional manifolds [9], among them nodal planes [59,92-95]. As we have seen above, the combination of a twofold screw rotation with time-reversal symmetry is one mechanism to enforce nodal planes via a generalized Kramers theorem. For example, a nodal plane is enforced in SG 76, where the combined antiunitary symmetry $2_{001}\left(0,0, \frac{1}{2}\right) \mathcal{T}$ leaves points in the $k_{z}=0$ and $\pi$ plane invariant and squares to a full lattice translation $t(0,0,1)$. In the $k_{z}=\pi$ plane, the translation eigenvalue is -1 and the condition for Kramers theorem is fulfilled [cf. Eq. (3.1)]. All

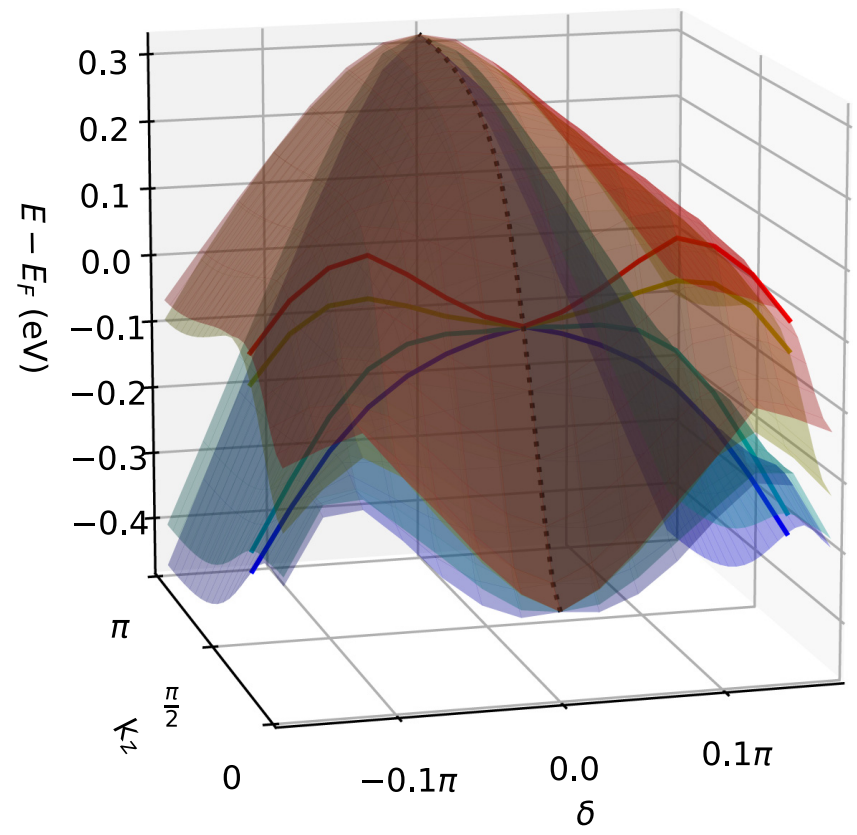

FIG. 12. Dispersion around the fourfold nodal line in $\mathrm{NaSn}_{5}$ in the plane defined by $\mathbf{k}=\left(\pi+\delta, \pi+\delta, k_{z}\right)$ in the vicinity of the line M-A. The dashed line highlights the fourfold degeneracy on the high-symmetry axis. The colored solid lines show the dispersion perpendicular to the nodal line at constant $k_{z}$, where the nodal line crosses the Fermi level.

such nodal planes are listed in Table I in the column "nodal planes."

Recently, nodal planes have been discussed in the context of topology [93,94,96], as they can also act as sources and sinks for Berry curvature. Within the tetragonal space groups there are three cases, SGs 92, 94, and 96, of enforced nodal planes, which additionally must have a topological charge, i.e., they act as source or sink of Berry flux [96] to balance the topological charges of symmetry-enforced Weyl points in the interior of the BZ.

\section{A. Space groups 92 and 96}

The SGs 92 and 96 form an enantiomorphic pair, i.e., they are mirrored versions of each other. They are generated from translations of the primitive lattice and the screw rotations $4_{001}\left(\frac{1}{2}, \frac{1}{2}, \frac{c}{4}\right)$ and $2010\left(\frac{1}{2}, \frac{1}{2}, \frac{c}{4}\right), c=1$ and 3 , respectively. The fourfold screw rotation squares to a twofold screw rotation, which means these two space groups have twofold screw rotations along 100, 010, and 001. With time-reversal symmetry present, all planes on the surface of the $\mathrm{BZ}$ are therefore Kramers degenerate, and the nodal planes form a closed box. Formally, there is a twofold degeneracy for every $\mathbf{k}$ with at least one component $k_{i}=\pi, i \in\{x, y, z\}$. To calculate the topological charge of this box, it can be enclosed by the surface of a slightly smaller box within because bands are nondegenerate in the interior of the BZ. This smaller box is simultaneously enclosing all band degeneracies in the interior when inverting the surface normal. Thus, the topological charge of the nodal planes equals the negative sum of all topological charges in the interior, which close the same gap 
as the nodal surface in question. Consequently, we have to consider the band connectivity across the whole BZ. Bands in these band structures appear always as multiples of eight with a certain minimum number of crossings (see Fig. 6). As shown above, the nonsymmorphic rotations enforce accordion and hourglass states, i.e., Weyl points at $\Gamma$ and movable topological crossings along the high-symmetry lines $\Gamma-\mathrm{X}$ and $\Gamma-Z$ (cf. Sec. IV). Although various symmetry-enforced band crossings appear in SGs 92 and 96, all topological charges in the interior can not add up to zero for any odd number of bands above (or equivalently below) the gap. In this case, there is always an enforced Weyl point at $\Gamma$ with a charge of \pm 1 , whereas the movable band crossings on the rotation axis close the gap in-between even sets of bands [see Figs. 4(a) and 4(c)]. Additional accidental band crossings might exist on the rotation axes, but they will have a multiplicity of two if they are on the line $\Gamma-\mathrm{Z}$ or four on the other two high-symmetry axes $\Gamma$-X and $\Gamma$-M. Since Weyl points with higher multiplicity are always symmetry related by rotations in these SGs, symmetryrelated copies will always have the same charge. Thus, the total charge in the interior of the BZ remains odd and the nodal plane carries an odd nonzero topological charge, which cannot be removed without breaking symmetries [96]. Despite this nonzero Chern number, any surface termination will overlap the nodal plane with its corresponding Weyl points such that, in this case, no Fermi arcs are associated with the topological nodal plane.

\section{Material example: $\mathrm{Ba}_{3} \mathrm{Sn}_{2}$}

As discussed in Sec. IV C, $\mathrm{Ba}_{3} \mathrm{Sn}_{2}$ crystallizes in SG 96 (or its enantiomorphic pair, SG 92). The highest occupied bands are clearly spin split, due to strong spin-orbit coupling [see Fig. 13(f)]. Figure 6 compares ab initio calculations with the sketch made from connectivity of irreducible representations. The enforced nodal plane can be seen as twofold degeneracy on all paths on the BZ surface for any band index. Due to the multiplicity of accidental crossings the enforced KramersWeyl point at $\Gamma$ is necessarily compensated by the topological charge of the nodal plane.

\section{B. Space group 94}

Space group 94 is special since only two surfaces are nodal planes and their charge is even and nonvanishing, i.e., at least \pm 2 . In contrast to the above, the fourfold screw rotation $4_{001}\left(\frac{1}{2}, \frac{1}{2}, \frac{1}{2}\right)$ in SG 94 squares to a symmorphic twofold rotation. Thus, the plane $k_{z}=\pi$ is not a nodal plane and the nodal manifold is restricted to the $k_{x}=\pi$ and $k_{y}=\pi$ planes. There is a conceptual difference to SGs discussed in [96] because SG 94 hosts Weyl points at the two TRIMs $\Gamma$ and $\mathrm{Z}$. However, they cannot balance their charges without additional crossings, the connectivity of bands requires that the Weyl point at $\mathrm{Z}$ has the same charge as the one at $\Gamma$, as in Fig. 4(b) [40]. Accidental crossings along $\Gamma-Z$ may occur in time-reversal related pairs but they cannot compensate the chirality, because the total phase picked up with band exchanges is restricted by the periodicity of the BZ [97]. As a result, the smallest possible change from these additional crossings on the rotation axis $\Gamma-\mathrm{Z}$ is \pm 4 . Away from the rotation axis and the nodal planes, any Weyl point appears at least four times with equal chirality, such that the nodal charge of the surface needs to be $\mathcal{C}=2+4 z, z \in \mathbb{Z}$. On a (001) surface in SG 94, two Fermi arcs must connect the projected Weyl points from $\Gamma$ and $\mathrm{Z}$ to the sides of the surface BZ.

\section{ACCIDENTAL BAND CROSSINGS/OFF-CENTERED SYMMETRIES}

Notwithstanding crossings protected by topological charges, symmetry-allowed perturbations to a given Hamiltonian may generally gap any crossing between bands unless they can be labeled by different symmetry eigenvalues. A specific realization of topological semimetallic phases may also contain accidental band crossings, and we briefly discuss such cases here. The dimensions of irreducible representations are comprehensively addressed in the literature $[63,64]$. Therefore, we restrict this discussion to a selection of these results and short remarks for the tetragonal space groups.

In space groups without inversion (Table I), eigenvalues are unpaired by default and crossings may appear at any $k$ with a symmetry beside translations in its little group. Exceptions can be the result of the nodal planes and certain rotation axes in mirror planes. Conversely, for inversion $\mathcal{P}$ and time reversal $\mathcal{T}$ the combination $\mathcal{P} \mathcal{T}$ pairs eigenvalues, such that twofold symmetries may not lead to accidental band crossings anymore. The only exceptions to this are off-centered symmetries [39].

Off-centered rotation (mirror) symmetries are characterized by translations perpendicular to the rotation axis (mirror plane). In real space they can be understood as having a different center than inversion, i.e., their sets of invariant points do not overlap. When the off-centered symmetry is a screw or glide operation it will enforce an odd number of point or line crossings, respectively. Hereby, the defining offcentered translation parts of the symmetries lead to a pairing of identical eigenvalues. Whereas nodal lines enforced by off-centered mirror symmetries are generally movable on the mirror plane, in the tetragonal space groups they are pinned to high-symmetry lines. They explain the existence of "Dirac lines" listed in Table II. Note that point crossings due to off-centered symmetries are always pinned by time-reversal symmetry to TRIMs. They are included with other Dirac points in the column "Dirac points."

Finally, we list all possible sets of $k$ points with accidental crossings in the columns "accidental points" and "accidental lines," which follow from the off-centered symmetries. With knowledge of accidental nodal points and lines, it is evident whether a crossing along a high-symmetry path is solely a Dirac point or part of a nodal line.

\section{CONCLUSIONS}

In conclusion, we classified all possible symmetryenforced band crossings in tetragonal materials with strong spin-orbit coupling. We considered both movable and pinned band degeneracies. We uncovered a rich variety of topological band crossings, which arise due to the intricate interplay of symmetry and topology (Tables I and II). This includes different types of pinned and movable Weyl points, specifically, single and double Weyl points and fourfold double and quadruple Weyl points (Sec. IV and Fig. 5). 
Usually, these Weyl points come in multiple copies that are related by symmetry (i.e., they have large multiplicity). However, for SGs 119 and 120 with body-centered unit cells we found that there can exist Weyl points at TRIMs that have only one symmetry-related partner (i.e., multiplicity two). This may be important for applications since materials with fewer Weyl points could exhibit simpler and more pronounced topological responses. For example, if time-reversal symmetry is broken, the anomalous Hall conductivity is expected to be enhanced since it scales with the distance between the Weyl points, which in SGs 119 and 120 is more than half a reciprocal lattice vector. Moreover, the anomalous Hall conductivity tensor has more nonzero entries than in other SGs with more Weyl points since contributions from different Weyl pairs do not cancel. That being said, in SGs 119 and 120 the Fermi surface pockets at the TRIMs without Weyl points can also play a part in the topological responses, which may partially cancel the contributions from the Weyl points. This depends on the detailed band dispersions and must be studied for each given material in SGs 119 and 120 separately.

We also classified all possible symmetry-enforced Dirac points, which can be movable or pinned, in inversionsymmetric tetragonal systems (Sec. V). Interestingly, SGs 130, 133, and 138 have movable Dirac points with an hourglass dispersion (Fig. 7). SG 138 also has a symmetryenforced weak $\mathbb{Z}_{2}$ invariant leading to Dirac surface states. Similar features are expected in orthorhombic systems [45].

Nodal lines also exist in different varieties. We catalogued all possible symmetry-enforced Dirac nodal lines, and twofold and fourfold Weyl nodal lines (Secs. VI and VII). In SGs 102, 104, 109, 118, and 122 the Weyl nodal lines form chains off connected rings, i.e., nodal chains (Fig. 10), while in SG 110 they form an armillary sphere (Fig. 11). Interestingly, in SG 110 the global band topology allows, in principle, for a band structure with only the armillary sphere at the Fermi energy. The low-energy physics of a material with this property would be dominated by the nontrivial topology of the armillary sphere, which could prove useful for applications.

Finally, we investigated nodal planes whose existence is enforced by the combination of screw rotations with time reversal (Sec. VIII). Remarkably, in SGs 92, 94, and 96 the symmetries enforce a nontrivial topological charge of the nodal planes. This charge is compensated by a single Weyl point at $\Gamma$ for SGs 92 and 96 . SG 94 is particularly interesting since it has a nodal plane duo (as opposed to a nodal plane trio in SGs 92 and 96) with arc surface states connecting two Weyl points with equal chirality at $\Gamma$ and $\mathrm{Z}$ to the nodal planes.

We emphasize that all of the aforementioned band degeneracies are purely symmetry enforced. That is, they occur in all bands of all materials crystallizing in the given SG, regardless of the chemical composition and other material details. These symmetry-enforced band degeneracies cannot be annihilated by any symmetry-preserving perturbation. This is in contrast to accidental band degeneracies which can be pair annihilated and which are only perturbatively stable.

Using our classification Tables I and II it is now possible to specifically design (meta)materials with the desired band topologies. It is also possible to search for existing compounds with these topological band crossings using materials databases, such as the ICSD. We performed such a search and found seven candidate materials (Fig. 2). Particularly interesting are the compounds where the band degeneracies cross the Fermi level, which is the case for the twofold and fourfold Weyl nodal lines in $\mathrm{Ba}_{5} \mathrm{In}_{4} \mathrm{Bi}_{5}$ and $\mathrm{NaSn}_{5}$, respectively. We hope that our findings will stimulate experimentalists to synthesize and characterize these materials and to design new (meta)materials based on our classifications.

Directions for future research include the classification of band crossings in the magnetic subgroups of the tetragonal SGs, as well as the study of band crossings in tetragonal systems without spin-orbit coupling. Without spin-orbit coupling the number and types of topological band crossings is much smaller. But, some nonsymmorphic symmetries, possibly in combination with time-reversal symmetry, can still lead to extra degeneracies. For example, the nodal planes, which are protected by the product of time reversal and a screw rotation, are still present in the case of no spin-orbit coupling $[97,98]$.

\section{ACKNOWLEDGMENTS}

The authors thank C. Ast, C.-K. Chiu, L. M. Schoop, M. G. Vergniory, and A. Yaresko for useful discussions. This research was supported in part by the National Science Foundation under Grant No. NSF PHY-1748958. D.H.F. gratefully acknowledges financial support from the Alexander von Humboldt Foundation.

\section{APPENDIX A: ADDITIONAL BAND-STRUCTURE CALCULATIONS}

In Fig. 13 we present the band-structure calculations along standard high-symmetry paths [48] for the example materials selected according to the selection criteria mentioned in Sec. II B and studied in the main text. Four of these are metallic with the topological features close to the Fermi level. Of the gapped examples, $\mathrm{AuBr}$ and $\mathrm{Ba}_{3} \mathrm{Sn}_{2}$ have a large gap and serve only to illustrate the features of their corresponding space groups.

\section{APPENDIX B: GENERIC MODELS AND QUALITATIVE DESCRIPTION OF SURFACE STATES}

The arguments of the main text yield the enforced features for any material realization. Yet, in real materials the features can not always be resolved and a tight-binding model exhibiting sufficiently large spin-orbit coupling is more suitable to point out the enforced behavior.

We derive generic models by placing $s$ orbitals with a spin degree of freedom on a Wyckoff position with lowest multiplicity, considering hopping from one site to the others up to a sufficiently large cutoff distance, and obtain all symmetry-related terms. Thereby symmetry-forbidden terms vanish and all exchange terms including spin are treated on the same footing. Finally, random values for the remaining hopping amplitudes are used to evaluate the model. Numeric calculations of Chern numbers and chiralities are performed by the Wilson loop method [99].

In the following sections we denote the high-symmetry points of the surface $\mathrm{BZ}$ by one of the respective bulk positions, which are projected onto it. A bar is added to 

(a) $\mathrm{Hf}_{3} \mathrm{Sb}$
(b) $\mathrm{Cs}_{2} \mathrm{Tl}_{3}$
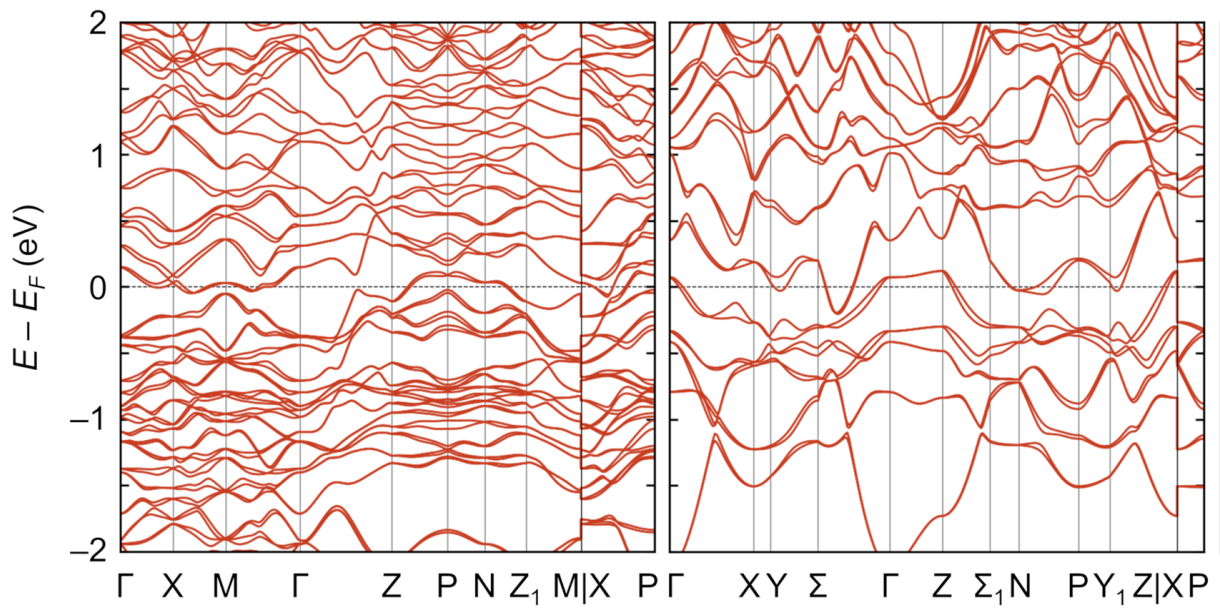

(d) $\mathrm{AuBr}$

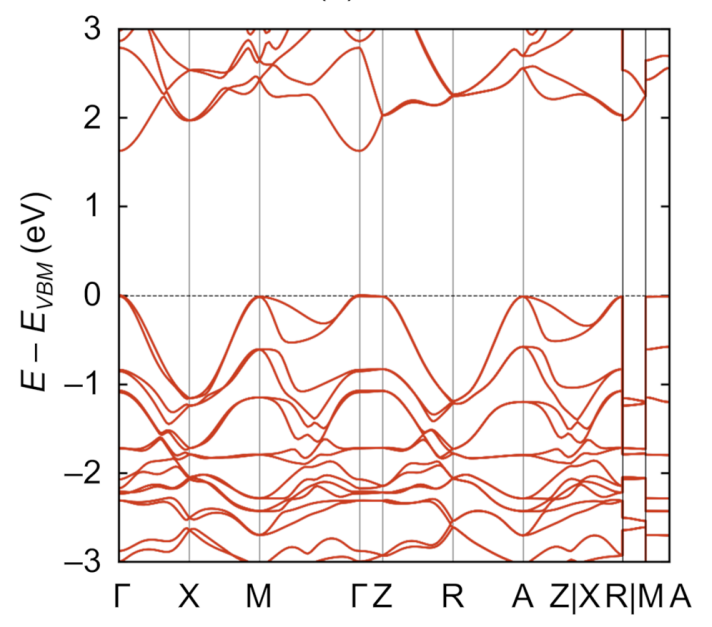

(f) $\mathrm{Ba}_{3} \mathrm{Sn}_{2}$

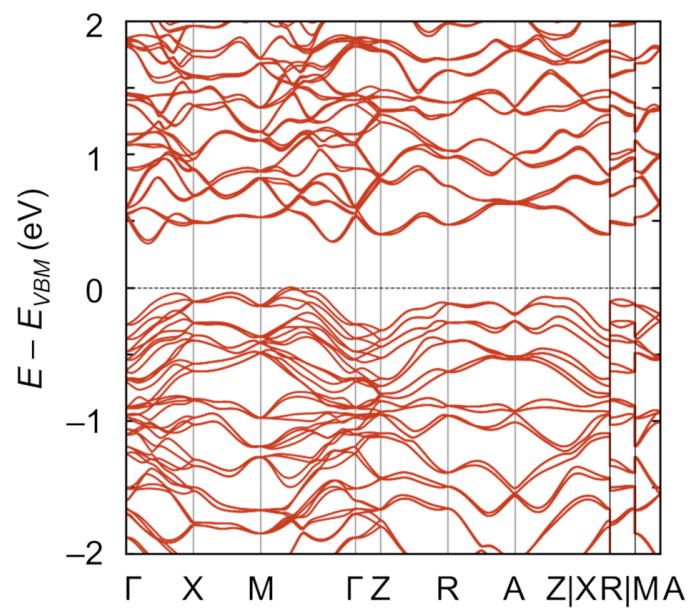

(c) $\mathrm{Ba}_{5} \mathrm{In}_{4} \mathrm{Bi}_{5}$

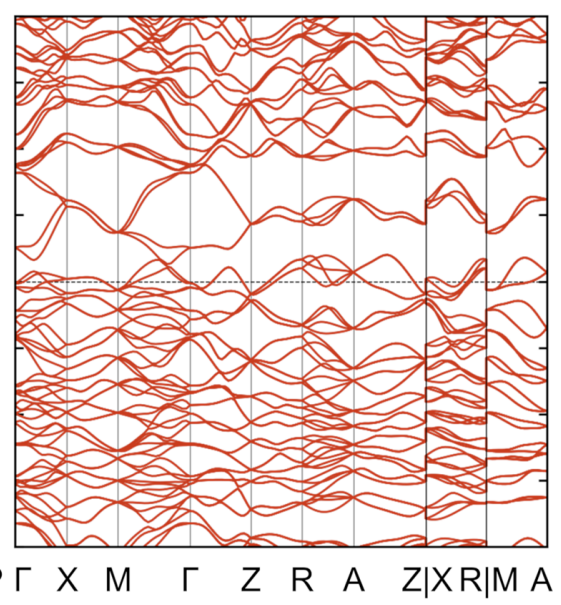

(e) $\mathrm{Tl}_{4} \mathrm{PbSe}_{3}$

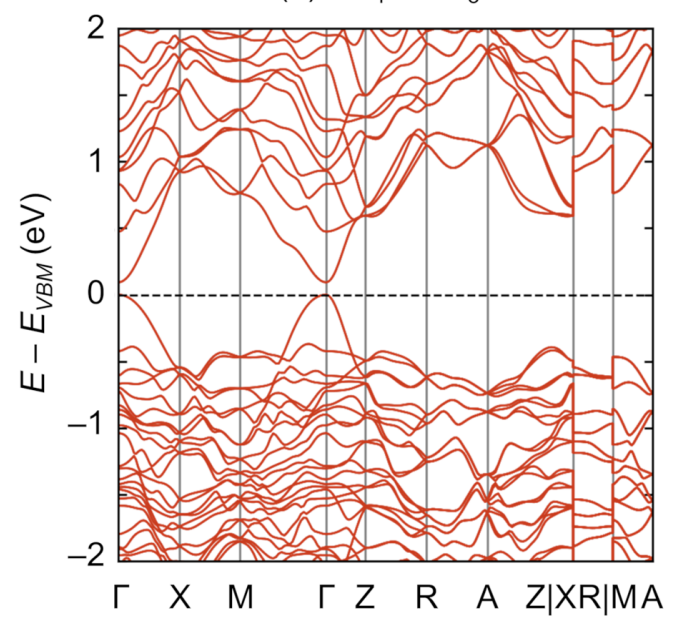

(g) $\mathrm{NaSn}_{5}$

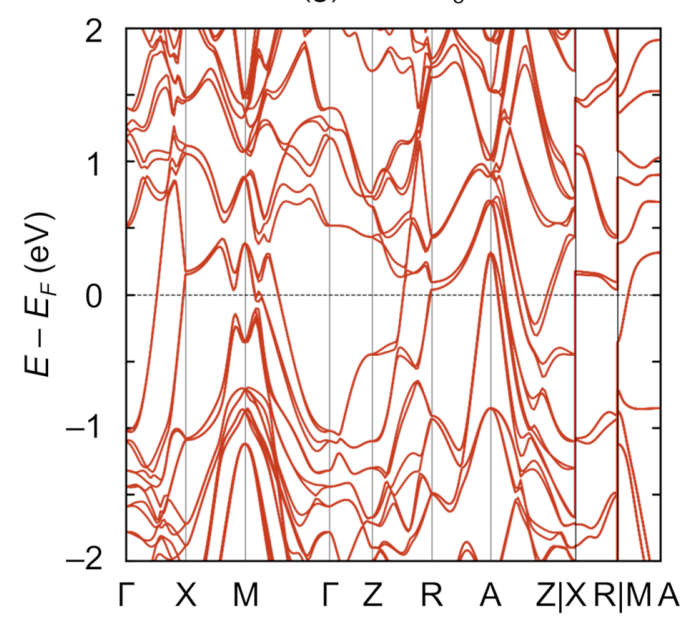

FIG. 13. DFT band structures of the examples presented in the main text. Detail figures of the relevant sections are shown in the corresponding chapters.

differentiate the labels, e.g., $\Gamma$ becomes $\bar{\Gamma}$ for any surface termination. Figures 14, 15, and 16 represent the surface states of a slab with 30 or more layers at a cut at constant energy through the spectrum. At each $k$ point the color is determined by the expectation value $\left\langle P_{r}\right\rangle_{\psi}$ of the projection operator $P_{r}$ on the surface, where $\psi$ is the eigenstate that gives the largest value of $\left\langle P_{r}\right\rangle_{\psi}$. The surface is defined to be $10 \%$ of the full slab thickness. 


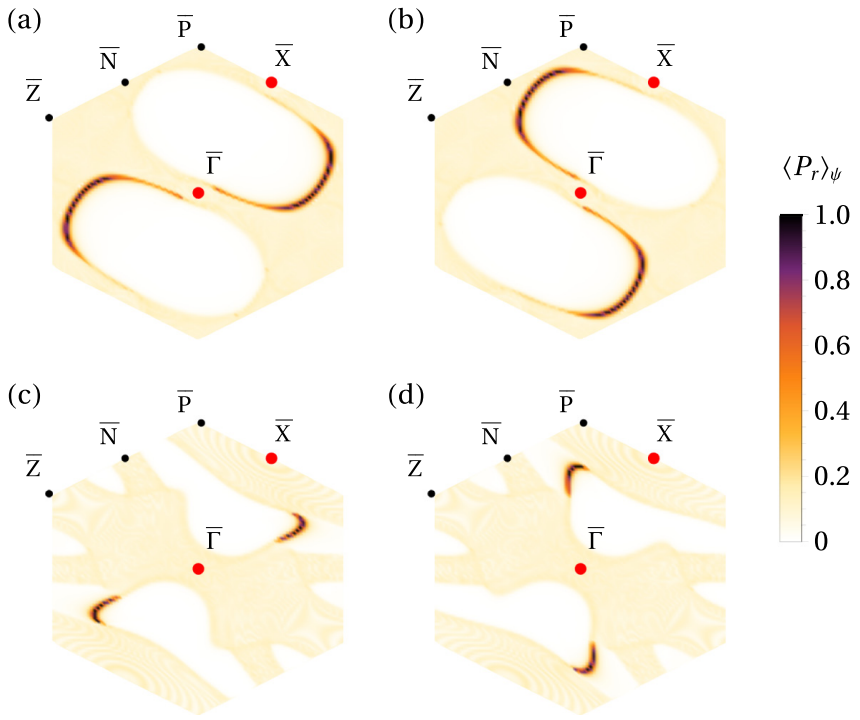

FIG. 14. Surface states from SG 119 for two generic models. The slab is $(1 \overline{1} 0)$ terminated with a chemical potential chosen in the vicinity of the Kramers-Weyl points. Red disks mark the projections of the only Weyl points of the respective model. In (a) and (b) [(c) and (d)] the model contains up to second- (third-)-nearest-neighbor hopping. (a), (c) Show the top and (b), (d) the bottom surfaces.

\section{Minimal number of Kramers points: SG 119}

SG 119 (and 120) exhibits the minimal number of two Weyl points in the absence of accidental crossings. To confirm this assessment, we devise generic models as described above with second- and third-nearest-neighbor hopping without accidental features. We then confirm the chirality at the point $\mathrm{X}$ to be $\mathcal{C}= \pm 1$ and determine the surface states for the (110) termination (see Fig. 14). Indeed, Fermi arcs connect the projections of the Weyl points and attach to the bulk bands close to the expected positions $\bar{\Gamma}$ and $\bar{X}$.

When calculating the Chern number on any plane spanned by [001] and a linear combination of [100] and [010] one does not enclose one of the Kramers-Weyl points and thus obtains zero. This is due to the connectivity of the body-centered BZ (here $\mathrm{BCT}_{1}$ ). We find that lines parallel to $\bar{\Gamma}-\overline{\mathrm{Z}}$ cross both Fermi arcs (or the bulk bands close the surface gap) (see (a)

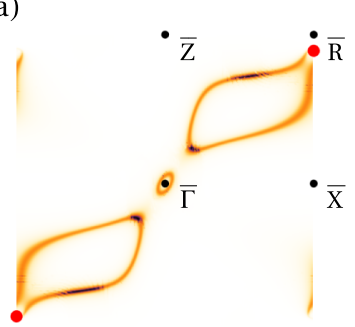

(b)

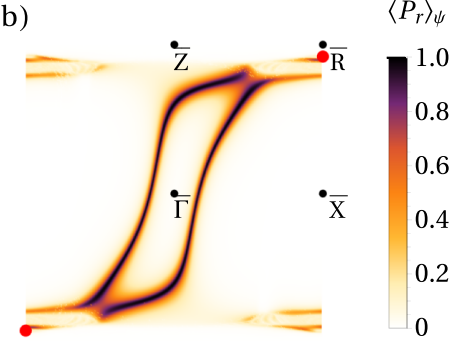

FIG. 15. Surface states of SGs 106 (a) and 133 (b). The energy cut is in the respective vicinity to the fourfold crossings. Both slabs are (100) terminated and calculated for models with up to fourthnearest-neighbor hopping. Red disks mark the projections of the two movable fourfold crossings for each model. Vanishing Chern numbers allow the hybridization of surface states.
Fig. 14). If one assigns a direction to the arcs, e.g., both can be considered to start at $\bar{\Gamma}$ and end at $\bar{X}$, then they cross the line parallel to $\bar{\Gamma}-\bar{Z}$ in opposite directions in accordance with the vanishing Chern number on the corresponding bulk surface. The structure of Fermi arcs thereby respects the twofold rotation symmetry $2_{1 \overline{1} 0}$ of the slab and the surfaces in Figs. 14(a) and 14(b) are related by 2001 .

The termination is chosen such that the two opposite chiralities at $\mathrm{X}$, which are related by the fourfold rotoinversion $\overline{4}_{001}$, are projected onto two distinct points of the surface BZ, which are labeled by $\bar{\Gamma}$ and $\bar{X}$. Simpler terminations like (100) or (001) map both $\mathrm{X}$ points on top of each other and the Fermi arc could not be observed.

Kramers theorem pairs opposite mirror eigenvalues at the TRIM N. As we have described in Sec. VI A, the crossing of mirror eigenvalues at $\mathrm{N}$ leads to almost movable nodal lines in SG 119. Although we focus on surface states, their existence is recognizable in bulk states shown in Fig. 14. For both models the nodal lines have the qualitative shape illustrated in Fig. 9(b). Whereas they appear as lines through $\mathrm{N}$ at constant $k_{z}$ for the parameters chosen in Figs. 14(a) and 14(b), they cross roughly at the midpoint of $\Gamma-Z$ for the model used in in Figs. 14(c) and 14(d). Especially in the second case the bulk bands crossing $\overline{\mathrm{N}}$ evidently belong to the almost movable nodal line. Its drumhead surface state is not visible at the chemical potential chosen in Fig. 14.

Challenges for a material realization are highlighted by the two different models shown in Fig. 14. The line $\Gamma$-Z-M hosts twofold degenerate bulk bands, i.e., they close the bulk gap in which the Fermi arcs appear. Thus, along the lines $\bar{\Gamma}-\bar{Z}$ and $\overline{\mathrm{X}}-\overline{\mathrm{P}}$ bulk bands overlap the projection of both Weyl points at

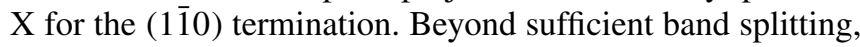
it is advantageous if the twofold degeneracy at the $\Gamma$ and $M$ points is at similar energy compared to the $\mathrm{X}$ point.

\section{Movable fourfold points: SGs 106 and 133}

For SGs 106 and 133 there is no chirality associated with the fourfold crossing on the M-A line due to the mirror symmetries. Yet, these semimetals generally have surface states because they consist of two superimposed Weyl semimetals. This can be understood by Weyl semimetal precursors that are distinguished by the action of the rotation symmetry $4_{001}\left(0,0, \frac{1}{2}\right)$. The screw rotation alone yields two unrelated representations, which each contain one Weyl point along the M-A line. Adding time reversal and the mirror symmetries yields the fourfold crossings, which are Dirac points for SG 133. The mirror symmetry relates the opposite chiralities and surface states.

With our generic models we confirm the presence surface states in SGs 106 and 133 (see Fig. 15). As expected for semimetals the surface states lie in the simplest case within a bulk gap. Whereas in principle the surface states look similar for SGs 106 and 133, we display them in two different variations. Figure 15(a) shows intersecting surface states, which hybridize close to $\bar{\Gamma}$ and split into three disjoint Fermi surfaces. In Fig. 15(b) the surface states do not intersect and only meet close to the surface projection of the fourfold crossing marked in red. At a chemical potential closer to the energy of the fourfold crossing the surface states gap out as well. 

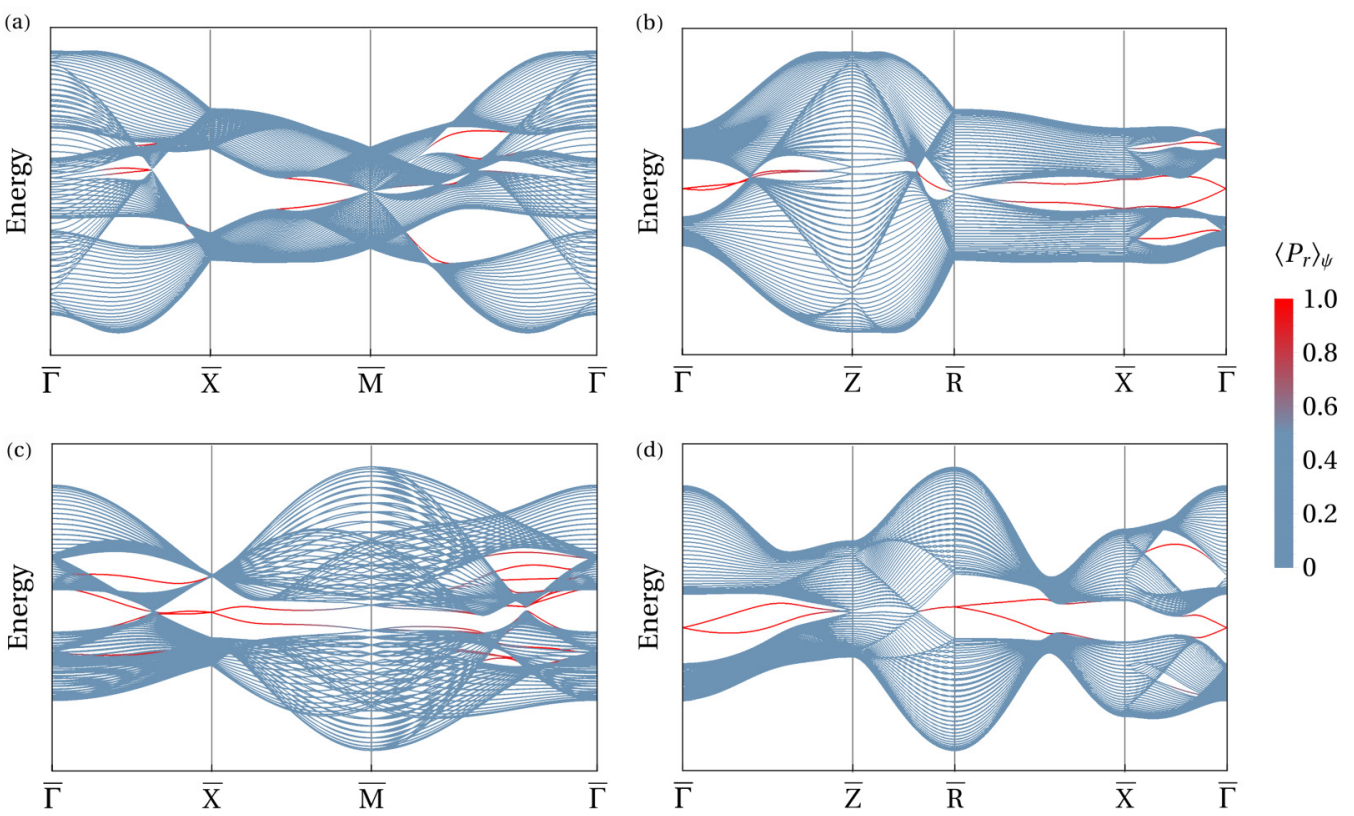

FIG. 16. Band structure for SGs 130 (a), (b) and SG 138 (c), (d). Two generic models with up to seventh-nearest-neighbor hopping are truncated and the surface localization is color coded. In the left column (a), (c) the termination (001) is chosen. For the right column (b), (d) the model is (100) terminated.

In conclusion, the surface arcs split into Fermi pockets which attach either twice to a fourfold crossing or are disconnected from it. Depending on the details of the model, intersections lead to regions of energetically flat surface bands.

\section{Movable Dirac points: SGs 130 and 138}

SGs 130 and 138 exhibit four movable Dirac points in the bulk on the line Z-R, whereas only SG 130 exhibits an additional eightfold crossing at the point $\mathrm{A}$. There are no other enforced crossings at the considered filling of $4+8 \mathbb{N}$, which translates into a half-filling for the slab calculations in Fig. 16. In the following we discuss the arrangement of inversion eigenvalues in our models and their surface states for (001) and (100) terminations.

As discussed in the main text, the product $\delta_{\Gamma_{i}}$ of every other occupied inversion eigenvalue at a TRIM $\Gamma_{i}$ is determined by the symmetries except for the center of the BZ, $\Gamma$. For our specific models of SGs 130 and 138 we obtain $\delta_{\Gamma}=+1$, the same value that is enforced by SG 138 at A, i.e., $\delta_{\mathrm{A}}=+1$. All other TRIMs yield $\delta_{\mathrm{X}}=\delta_{\mathrm{R}}=\delta_{\mathrm{M}}=\delta_{\mathrm{Z}}=-1$. We group the next arguments by the chosen termination, which determines how the $\delta_{\Gamma_{i}}$ must be combined to obtain the relevant timereversal polarization [80].

If a bulk system with SG 130 or 138 is terminated in (001) direction, the time-reversal polarizations are $\pi_{\bar{\Gamma}}=\pi_{\overline{\mathrm{M}}}=-1$ (if applicable) and $\pi_{\overline{\mathrm{X}}}=1$, which we label by the TRIMs of the surface BZ. For SG 130 [see Fig. 16(a)], the model exhibits a bulk band touching at the projection of the eightfold crossing at $\overline{\mathrm{M}}$. With this caveat in mind, the differences in the time-reversal polarization do not lead to characteristic surface states on the line $\overline{\mathrm{X}}-\overline{\mathrm{M}}$. Also, no feature related to a change in $\pi_{a}$ is expected along $\bar{\Gamma}-\bar{X}$ because the bulk Dirac point on Z-R closes the band gap. On this path we find states asso- ciated with the movable Dirac point emerging from the bulk crossing. Due to the vanishing Chern number, these surface states are not protected. Using a different parameter set, an even number of gapped states were achieved at $\bar{X}$ but the band connectivity is not affected. For SG 138, a nontrivial enforced surface state appears on $\overline{\mathrm{X}}-\overline{\mathrm{M}}$ shown in Fig. 16(c). The Dirac surface state at $\bar{X}$ exhibits the characteristic connections to valence and conduction bands.

For the (100) termination we need to consider the timereversal polarizations $\pi_{\bar{\Gamma}}=\pi_{\overline{\mathrm{R}}}=-1$ and $\pi_{\overline{\mathrm{X}}}=\pi_{\overline{\mathrm{Z}}}=+1$. Note that in the presence of the movable Dirac points on Z-R, $\pi_{\overline{\mathrm{Z}}}$ cannot be rigorously used, whereas yet again $\pi_{\overline{\mathrm{R}}}$ is not defined for SG 130 due to its eightfold crossing at A. For SGs 130 and 138 the values of $\pi_{a}$ change along $\bar{\Gamma}-\bar{X}$ and we find the surface Dirac point with the expected connectivity at $\bar{\Gamma}$ [see Figs. 16(b) and 16(d)]. The same happens for SG 138 along $\bar{X}-\bar{R}$, with the key difference that here this feature is enforced. Notably, whereas all bands are twofold degenerate by the action of $\mathcal{P} \mathcal{T}$, the surface states on the line $\bar{Z}-\bar{R}$ are Kramers degenerate due to $M_{010}\left(0, \frac{1}{2}, \frac{1}{2}\right) \mathcal{T}$. The latter is only true in the limit of large slabs, where the states localized on opposing surfaces cannot interact with each other.

In the discussion of surface states for SGs 106 and 133 as well as SGs 130 and 138 one notices that the former tend to resemble Fermi arcs more closely. We briefly try to rationalize this observation. For the latter SGs the interpretation as two different superimposed Weyl semimetals does not apply to the same extent as to the former SGs. A possible explanation for this is that on the line Z-R only one spinless representation exists for SGs 130 and 138, whereas there are two on the fourfold rotation axis M-A, where the fourfold crossing appears for SGs 106 and 133. This and the absence of surface states due to the $\mathbb{Z}_{2}$ invariant underlines the varying extent to which Fermi arcs vanish in our generic models. 


\section{APPENDIX C: EFFECTIVE LOW-ENERGY HAMILTONIANS}

In this Appendix we derive effective Hamiltonians, describing the low-energy physics near different types of band crossings. Throughout this chapter, we use coordinates $\mathbf{q}=$ $\mathbf{k}-\mathbf{K}_{0}$ relative to the point $\mathbf{K}_{0}$ of the degeneracy. The Hamiltonian is then given up to the lowest order in $\mathbf{q}$ such that only enforced degeneracies remain.

For all the spatial symmetries in the little group of $\mathbf{K}_{0}$, the Hamiltonian has to fulfill the condition $U_{i}^{\dagger} H(\mathbf{q}) U_{i}=H\left(R_{i} \mathbf{q}\right)$, where $U_{i}$ is the representation of the spatial symmetry and $R_{i}$ the action of the symmetry in $k$ space, i.e., the spatial symmetry without translational parts modulo reciprocal lattice vectors.

If antiunitary symmetries like time reversal are present, their representation can be given in terms of a unitary part $U_{\tilde{\mathcal{T}}}$ and complex conjugation $\mathcal{K}$. The condition on the local Hamiltonian is then $U_{\tilde{\mathcal{T}}}^{\dagger} H^{*}(\mathbf{q}) U=H\left(-R_{\tilde{\mathcal{T}}} \mathbf{q}\right)$.

\section{Fourfold double Weyl point at $\mathbf{R}$ in SG 96}

The little group of the TRIM $\mathrm{R}=(0, \pi, \pi)$ is generated from lattice translations and two of the three twofold screw rotations $2_{001}\left(0,0, \frac{1}{2}\right), 2_{010}\left(\frac{1}{2}, \frac{1}{2}, \frac{3}{4}\right)$, and $2_{100}\left(\frac{1}{2}, \frac{1}{2} \cdot \frac{1}{4}\right)$. The two-dimensional irreducible representation of these symmetries can be given by $\sigma_{z}, \sigma_{y}$, and $i \sigma_{x}$, respectively. Time-reversal symmetry couples two copies of these representations and takes the form $U_{\mathcal{T}} \mathcal{K}=i \tau_{y} \sigma_{z} \mathcal{K}$ in this basis. Here we write $\sigma_{i}, i=0, x, y, z$, for the Pauli matrices acting within a representation and $\tau_{i}$ for the Pauli matrices acting in the space of the two representations.

This restricts the linearized Hamiltonian to the form (in $\tau$ space)

$$
H(\mathbf{q})=\left(\begin{array}{cc}
\mathbf{d} \cdot \boldsymbol{\sigma} & \boldsymbol{\Lambda} \cdot \boldsymbol{\sigma} \\
\boldsymbol{\Lambda}^{*} \cdot \boldsymbol{\sigma} & \mathbf{b} \cdot \boldsymbol{\sigma}
\end{array}\right),
$$

with $d_{i}=v_{i} q_{i}, i=x, y, z$, and $b_{x}=d_{x}, b_{y}=-d_{y}, b_{z}=-d_{z}$. Furthermore, $\Lambda_{i}=\lambda_{i} q_{i}$ for $i=y, z$ and 0 otherwise.

Using the unitary transformation $U=\frac{1}{\sqrt{2}}\left(\tau_{z} \sigma_{0}+\tau_{y} \sigma_{x}\right)$, the Hamiltonian becomes block diagonal with two decoupled Weyl points mentioned in the main text and shown in Fig. 5(b):

$$
H_{ \pm}(\mathbf{q})=v_{x} q_{x} \sigma_{x}+\left( \pm v_{y} q_{y}+\lambda_{z} q_{z}\right) \sigma_{y}+\left( \pm v_{z} q_{z}-\lambda_{y} q_{y}\right) \sigma_{z} .
$$

The different signs have an influence on the dispersion only when $q_{z}$ and $q_{y}$ are both nonzero in accordance with requirement of the nodal planes $q_{z}=0$ and $q_{y}=0$.

\section{Low-energy Hamiltonian for fourfold quadruple Weyl point}

The point A is a TRIM and its little group consists of all the symmetries of the SG. Among the spinful irreducible representations, i.e., the ones with eigenvalue -1 for $2 \pi$ rotations, four are one dimensional and one is two dimensional [63]. The latter one is paired with itself by time-reversal symmetry and thus makes up the fourfold degeneracy.
This representation is completely defined for the following choice of matrices for the generators:

$$
\begin{aligned}
& 4_{001}^{+},\left(\frac{1}{2}, \frac{1}{2}, \frac{3}{4}\right): U_{4}=\tau_{0} \sigma_{z}, \\
& 2_{010},\left(\frac{1}{2}, \frac{1}{2}, \frac{3}{4}\right): U_{2}=\tau_{0} \sigma_{y} .
\end{aligned}
$$

With this basis choice, the unitary part of the time-reversal symmetry takes the form $U_{\mathcal{T}}=i \tau_{y} \sigma_{z}$.

Including all terms up to second order in $\mathbf{q}$ and comparing coefficients leads to the Hamiltonian

$$
H(\mathbf{q})=\left(\begin{array}{cc}
\mathbf{d}(\mathbf{q}) \cdot \boldsymbol{\sigma} & \boldsymbol{\Lambda}(\mathbf{q}) \cdot \boldsymbol{\sigma} \\
\boldsymbol{\Lambda}^{*}(\mathbf{q}) \cdot \boldsymbol{\sigma} & -\mathbf{d}(\mathbf{q}) \cdot \sigma^{*}
\end{array}\right),
$$

with the Hamiltonian of a double Weyl on the diagonal, $d_{x}=v_{x} q_{x} q_{y}, d_{y}=v_{y}\left(q_{x}^{2}-q_{y}^{2}\right), d_{z}=v_{z} q_{z}$, and the symmetryallowed spin-orbit coupling terms $\Lambda_{x}=-\lambda_{x} q_{x} q_{y}, \Lambda_{y}=0$, $\Lambda_{z}=\lambda_{z} q_{z}$. Note that $-\mathbf{d} \cdot \boldsymbol{\sigma}^{*}$ effectively inverts the signs of $v_{x}$ and $v_{z}$, leaving the Chern number of the block invariant.

The block-diagonal form in Eq. (4.5) in the main text can be achieved with the unitary transformation $U=\frac{1}{\sqrt{2}}\left(\tau_{0} \sigma_{0}+\right.$ $\left.i \tau_{x} \sigma_{y}\right)$. This matrix diagonalizes $i \tau_{y} \sigma_{y}$, which commutes with the initial Hamiltonian. Each block corresponds to a different eigenvalue of this symmetry, which has been used to color Fig. 5(c).

\section{Low-energy Hamiltonian for $P$ in SG 110}

The little group of P in SG 110 is generated by translations, including $t\left(\frac{1}{2}, \frac{1}{2}, \frac{1}{2}\right)$, the rotation $2_{001}\left(\frac{1}{2}, \frac{1}{2}, \frac{1}{2}\right)$, the glide reflection $M_{110}\left(0, \frac{1}{2}, \frac{3}{4}\right)$, and the combination of time reversal and another reflection $M_{010}\left(0,0, \frac{1}{2}\right) \mathcal{T}$.

From Eqs. (6.5) and (6.6) we know that different mirror eigenvalues are paired when both rotation eigenvalues are +1 , i.e., $p_{2_{001}}=0$ in (6.4). The representations can therefore readily be given as

$$
\begin{gathered}
U_{2_{001}}=\sigma_{0}, \\
U_{M_{110}}=i e^{i 3 \pi / 4} \sigma_{z} .
\end{gathered}
$$

In this basis, $M_{010}\left(0,0, \frac{1}{2}\right) \mathcal{T}$ takes the form $i \sigma_{y} \mathcal{K}$.

To fully recreate the gap in a low-energy model, it is necessary to go to third order in q. The Hamiltonian is restricted to the form

$H(\mathbf{q})=a\left(q_{x}^{2}-q_{y}^{2}\right) q_{z} \sigma_{+}+\left(c_{1} q_{z}+2 c_{2} q_{x} q_{y}\right) \sigma_{z}+$ H.c.,

with $\sigma_{+}=\sigma_{x}+i \sigma_{y}, a \in \mathbb{C}$, and $c_{i} \in \mathbb{R}$

To better show the behavior in the mirror planes, we introduce rotated coordinates $p_{-}=\frac{1}{\sqrt{2}}\left(q_{x}-q_{y}\right)$ and $p_{+}=$ $\frac{1}{\sqrt{2}}\left(q_{x}+q_{y}\right)$, which span together with $q_{z}$ the mirror planes $M_{110}$ and $M_{1 \overline{1} 0}$, respectively. With this, the Hamiltonian reads as

$$
H(\mathbf{q})=2 a p_{+} p_{-} q_{z} \sigma_{+}+\left[c_{1} q_{z}+c_{2}\left(p_{+}^{2}-p_{-}^{2}\right)\right] \sigma_{z}+\text { H.c. }
$$

This shows that within each mirror plane, only the prefactor of $\sigma_{z}$ is nonzero. Within this term, there is a $q_{z}$ for every $p_{ \pm}$such that this term vanishes as well, leading to the almost movable 


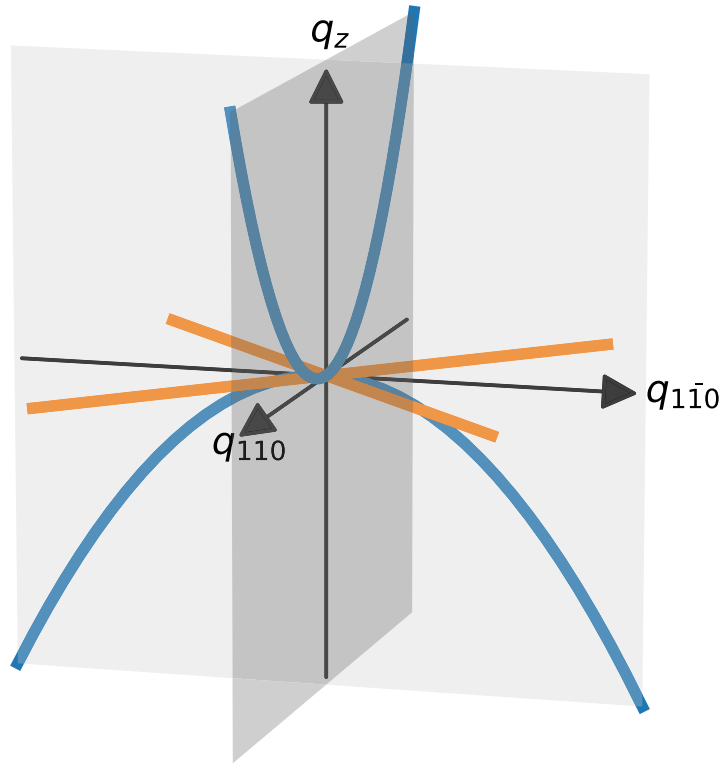

FIG. 17. Touching nodal lines at $\mathrm{P}$ in SG 110 . The orange lines are pinned to the path $\mathrm{P}-\mathrm{N}$, the blue lines are almost movable nodal lines each within a mirror plane. The latter result from the fact that the bands at $\mathrm{P}$ are formed by mirror eigenvalues of different signs (see Sec. VI A).

nodal line in each mirror plane as required by the exchange of mirror eigenvalues at $\mathrm{P}$, see Fig.17. Because of the restrictions for odd and even contributions for $q_{z}$ and $p_{ \pm}$, respectively, this still holds when including arbitrary higher-order terms. The nodal lines in both planes are furthermore symmetry related because of the constraint on the coefficient $c_{2}$ of $p_{+}$and $p_{-}$.

\section{Linearized Hamiltonian for fourfold Weyl line}

In this section we construct a linearized Hamiltonian for the fourfold nodal line on the M-A line in SGs 113 and 114. We follow the procedure of [49], where a linearized Hamiltonian for fourfold double Weyl points can be found. Note that only the directions perpendicular to the nodal line are expanded in relative coordinates $q_{x}$ and $q_{y}$, as $k_{z}$ spans the full range from $-\pi$ to $\pi$ along the nodal line.

The little group of a vector $\mathbf{k}=\left(\pi, \pi, k_{z}\right)$ is $C_{2 v}$, generated by $2_{001}$ and $M_{110}\left(\frac{1}{2}, \frac{1}{2}, c\right)$ and has two-dimensional spinful irreducible representations [63]. With time-reversal symmetry present, an additional generator $\overline{4}_{001}^{+} \mathcal{T}$ is included, which again relates two copies of the double group representation of $C_{2 v}$ and squares to -2001 . The other antiunitary symmetry mentioned in the main text, $2_{010}\left(\frac{1}{2}, \frac{1}{2}, c\right) \mathcal{T}$, can be written as product of the above generators.

A matrix representation can be given by

$$
\begin{gathered}
U_{2001}=\tau_{0}\left(-i \sigma_{y}\right), \\
U_{M_{110}}=\tau_{0}\left(i e^{i k_{z} c} \sigma_{x}\right), \\
U_{\overline{4}_{001}^{+} \mathcal{T}} \mathcal{K}=-i \tau_{y} \frac{\sigma_{0}-i \sigma_{y}}{\sqrt{2}} \mathcal{K} .
\end{gathered}
$$

The linearized Hamiltonian close to the nodal line at $\mathbf{q}=$ $\left(\pi+q_{x}, \pi+q_{y}, k_{z}\right)$ is restricted by these symmetries to the form

$$
H(\mathbf{q})=\left(\begin{array}{ll}
\alpha q_{+} \sigma_{z}+\beta q_{-} \sigma_{x} & \lambda\left(q_{+} \sigma_{z}-q_{-} \sigma_{x}\right) \\
\lambda\left(q_{+} \sigma_{z}-q_{-} \sigma_{x}\right) & \beta q_{+} \sigma_{z}+\alpha q_{-} \sigma_{x}
\end{array}\right)+\varepsilon_{0} \mathbb{1},
$$

with the perpendicular momentum components $q_{ \pm}=q_{x} \pm q_{y}$ and the parameters $\alpha, \beta, \lambda$, and $\varepsilon_{0}$ all being $k_{z}$ dependent without any restrictions.

This Hamiltonian can be block diagonalized via the unitary $q_{x^{-}}$and $q_{y}$-independent matrix $T=\cos \phi \tau_{0} \sigma_{0}+\sin \phi i \tau_{y} \sigma_{0}$, where $\tan (2 \phi)=\frac{2 \lambda}{\alpha-\beta}$. The block-diagonal Hamiltonian reads as

$$
H(\mathbf{q})=\left(\begin{array}{cc}
\tilde{\alpha} q_{+} \sigma_{z}-\tilde{\beta} q_{-} \sigma_{x} & 0 \\
0 & \tilde{\beta} q_{+} \sigma_{z}-\tilde{\alpha} q_{-} \sigma_{x}
\end{array}\right)+\varepsilon_{0} \mathbb{1},
$$

with two decoupled Weyl lines with the modified velocities

$$
\begin{aligned}
& \tilde{\alpha}=\frac{\alpha+\beta}{2}+\operatorname{sgn}(\alpha-\beta) \sqrt{\left(\frac{\alpha-\beta}{2}\right)+\lambda^{2}}, \\
& \tilde{\beta}=\frac{\alpha+\beta}{2}-\operatorname{sgn}(\alpha-\beta) \sqrt{\left(\frac{\alpha-\beta}{2}\right)+\lambda^{2}} .
\end{aligned}
$$

In the nodal planes we find $\left|q_{+}\right|=\left|q_{-}\right|$, leading to identical eigenvalues as demanded by symmetry. Furthermore, a $\frac{\pi}{2}$ rotation relates the eigenvalues of the upper block to the ones in the lower one and vice versa. The nodal line in each subspace is protected by a Berry phase of $\pi$. Including symmetryallowed terms of higher order in $\mathbf{q}$ adds an identical quadratic contribution to both bands, seen in the collective bending of bands in the dispersion of $\mathrm{NaSn}_{5}$.
[1] C.-K. Chiu, J. C. Y. Teo, A. P. Schnyder, and S. Ryu, Rev. Mod. Phys. 88, 035005 (2016).

[2] G. E. Volovik, Topology of Quantum Vacuum, Lecture Notes in Physics, Vol. 870 (Springer, Berlin, 2013), pp. 343-383.

[3] N. P. Armitage, E. J. Mele, and A. Vishwanath, Rev. Mod. Phys. 90, 015001 (2018).

[4] A. Burkov, Annu. Rev. Condens. Matter Phys. 9, 359 (2018).

[5] S.-Y. Yang, H. Yang, E. Derunova, S. S. P. Parkin, B. Yan, and M. N. Ali, Adv. Phys.: X 3, 1414631 (2018).

[6] J. Hu, S.-Y. Xu, N. Ni, and Z. Mao, Annu. Rev. Mater. Res. 49, 207 (2019).
[7] A. A. Burkov, Nat. Mater. 15, 1145 (2016).

[8] A.-Q. Wang, X.-G. Ye, D.-P. Yu, and Z.-M. Liao, ACS Nano 14, 3755 (2020).

[9] C. Herring, Phys. Rev. 52, 365 (1937).

[10] T. Bzdusek, Q. Wu, A. Rüegg, M. Sigrist, and A. A. Soluyanov, Nature (London) 538, 75 (2016).

[11] Q. Wang, Y. Xu, R. Lou, Z. Liu, M. Li, Y. Huang, D. Shen, H. Weng, S. Wang, and H. Lei, Nat. Commun. 9, 3681 (2018).

[12] B. Skinner and L. Fu, Sci. Adv. 4, eaat2621 (2018).

[13] X. Wan, A. M. Turner, A. Vishwanath, and S. Y. Savrasov, Phys. Rev. B 83, 205101 (2011). 
[14] A. A. Burkov, M. D. Hook, and L. Balents, Phys. Rev. B 84, 235126 (2011).

[15] S.-Y. Xu, C. Liu, S. K. Kushwaha, R. Sankar, J. W. Krizan, I. Belopolski, M. Neupane, G. Bian, N. Alidoust, T.-R. Chang, H.T. Jeng, C.-Y. Huang, W.-F. Tsai, H. Lin, P. P. Shibayev, F.-C. Chou, R. J. Cava, and M. Z. Hasan, Science 347, 294 (2015).

[16] S.-M. Huang, S.-Y. Xu, I. Belopolski, C.-C. Lee, G. Chang, B. Wang, N. Alidoust, G. Bian, M. Neupane, C. Zhang, S. Jia, A. Bansil, H. Lin, and M. Z. Hasan, Nat. Commun. 6, 7373 (2015).

[17] Y.-H. Chan, C.-K. Chiu, M. Y. Chou, and A. P. Schnyder, Phys. Rev. B 93, 205132 (2016).

[18] G. Bian, T.-R. Chang, R. Sankar, S.-Y. Xu, H. Zheng, T. Neupert, C.-K. Chiu, S.-M. Huang, G. Chang, I. Belopolski, D. S. Sanchez, M. Neupane, N. Alidoust, C. Liu, B. Wang, C.-C. Lee, H.-T. Jeng, C. Zhang, Z. Yuan, S. Jia et al., Nat. Commun. 7, 10556 (2016).

[19] G. Bian, T.-R. Chang, H. Zheng, S. Velury, S.-Y. Xu, T. Neupert, C.-K. Chiu, S.-M. Huang, D. S. Sanchez, I. Belopolski, N. Alidoust, P.-J. Chen, G. Chang, A. Bansil, H.-T. Jeng, H. Lin, and M. Z. Hasan, Phys. Rev. B 93, 121113(R) (2016).

[20] K. Fukushima, D. E. Kharzeev, and H. J. Warringa, Phys. Rev. D 78, 074033 (2008).

[21] M. M. Vazifeh and M. Franz, Phys. Rev. Lett. 111, 027201 (2013).

[22] P. Goswami, J. H. Pixley, and S. Das Sarma, Phys. Rev. B 92, 075205 (2015).

[23] X. Huang, L. Zhao, Y. Long, P. Wang, D. Chen, Z. Yang, H. Liang, M. Xue, H. Weng, Z. Fang, X. Dai, and G. Chen, Phys. Rev. X 5, 031023 (2015).

[24] P. Liu, J. R. Williams, and J. J. Cha, Nat. Rev. Mater. 4, 479 (2019).

[25] J. Tian, I. Miotkowski, S. Hong, and Y. P. Chen, Sci. Rep. 5, 14293 (2015).

[26] J. Tian, S. Hong, I. Miotkowski, S. Datta, and Y. P. Chen, Sci. Adv. 3, e1602531 (2017).

[27] P. Li, W. Wu, Y. Wen, C. Zhang, J. Zhang, S. Zhang, Z. Yu, S. A. Yang, A. Manchon, and X.-x. Zhang, Nat. Commun. 9, 3990 (2018).

[28] J. Kim, S. S. Baik, S. H. Ryu, Y. Sohn, S. Park, B.-G. Park, J. Denlinger, Y. Yi, H. J. Choi, and K. S. Kim, Science 349, 723 (2015).

[29] Q. Wang, C.-Z. Li, S. Ge, J.-G. Li, W. Lu, J. Lai, X. Liu, J. Ma, D.-P. Yu, Z.-M. Liao, and D. Sun, Nano Lett. 17, 834 (2017).

[30] M. Yang, J. Wang, J. Han, J. Ling, C. Ji, X. Kong, X. Liu, Z. Huang, J. Gou, Z. Liu, F. Xiu, and Y. Jiang, ACS Photonics 5, 3438 (2018).

[31] S. Chi, Z. Li, Y. Xie, Y. Zhao, Z. Wang, L. Li, H. Yu, G. Wang, H. Weng, H. Zhang, and J. Wang, Adv. Mater. 30, 1801372 (2018).

[32] R. Lundgren, P. Laurell, and G. A. Fiete, Phys. Rev. B 90, 165115 (2014).

[33] S. M. Young, S. Zaheer, J. C. Y. Teo, C. L. Kane, E. J. Mele, and A. M. Rappe, Phys. Rev. Lett. 108, 140405 (2012).

[34] L. M. Schoop, M. N. Ali, C. Straßer, A. Topp, A. Varykhalov, D. Marchenko, V. Duppel, S. S. P. Parkin, B. V. Lotsch, and C. R. Ast, Nat. Commun. 7, 11696 (2016).

[35] Y. X. Zhao and A. P. Schnyder, Phys. Rev. B 94, 195109 (2016).

[36] S. M. Young and C. L. Kane, Phys. Rev. Lett. 115, 126803 (2015).
[37] A. Furusaki, Sci. Bull. 62, 788 (2017).

[38] R. Takahashi, M. Hirayama, and S. Murakami, Phys. Rev. B 96, 155206 (2017).

[39] B.-J. Yang, T. A. Bojesen, T. Morimoto, and A. Furusaki, Phys. Rev. B 95, 075135 (2017).

[40] S. S. Tsirkin, I. Souza, and D. Vanderbilt, Phys. Rev. B 96, 045102 (2017).

[41] C. Fang, Y. Chen, H.-Y. Kee, and L. Fu, Phys. Rev. B 92, 081201(R) (2015).

[42] M. Malard, P. E. de Brito, S. Östlund, and H. Johannesson, Phys. Rev. B 98, 165127 (2018).

[43] J. Zhang, Y.-H. Chan, C.-K. Chiu, M. G. Vergniory, L. M. Schoop, and A. P. Schnyder, Phys. Rev. Mater. 2, 074201 (2018).

[44] Y.-H. Chan, B. Kilic, M. M. Hirschmann, C.-K. Chiu, L. M. Schoop, D. G. Joshi, and A. P. Schnyder, Phys. Rev. Mater. 3, 124204 (2019).

[45] A. Leonhardt (unpublished).

[46] H. Nielsen and M. Ninomiya, Phys. Lett. B 105, 219 (1981).

[47] H. Nielsen and M. Ninomiya, Nucl. Phys. B 185, 20 (1981).

[48] W. Setyawan and S. Curtarolo, Comput. Mater. Sci. 49, 299 (2010).

[49] F. Flicker, F. de Juan, B. Bradlyn, T. Morimoto, M. G. Vergniory, and A. G. Grushin, Phys. Rev. B 98, 155145 (2018).

[50] A. Jain, S. P. Ong, G. Hautier, W. Chen, W. D. Richards, S. Dacek, S. Cholia, D. Gunter, D. Skinner, G. Ceder, and K. a. Persson, APL Mater. 1, 011002 (2013).

[51] S. P. Ong, S. Cholia, A. Jain, M. Brafman, D. Gunter, G. Ceder, and K. A. Persson, Comput. Mater. Sci. 97, 209 (2015).

[52] https://icsd.fiz-karlsruhe.de.

[53] G. Kresse and J. Furthmüller, Phys. Rev. B 54, 11169 (1996).

[54] G. Kresse and J. Furthmüller, Comput. Mater. Sci. 6, 15 (1996).

[55] P. E. Blöchl, Phys. Rev. B 50, 17953 (1994).

[56] G. Kresse and D. Joubert, Phys. Rev. B 59, 1758 (1999).

[57] J. P. Perdew, K. Burke, and M. Ernzerhof, Phys. Rev. Lett. 77, 3865 (1996).

[58] E. Wigner, Nachrichten der Gesellschaft der Wissenschaften zu Göttingen Mathematisch-Physikalische Klasse 546, 559 (1932).

[59] G. Chang, B. J. Wieder, F. Schindler, D. S. Sanchez, I. Belopolski, S.-M. Huang, B. Singh, D. Wu, T.-R. Chang, T. Neupert et al., Nat. Mater. 17, 978 (2018).

[60] Z. Wang, D. Gresch, A. A. Soluyanov, W. Xie, S. Kushwaha, X. Dai, M. Troyer, R. J. Cava, and B. A. Bernevig, Phys. Rev. Lett. 117, 056805 (2016).

[61] K. Koepernik, D. Kasinathan, D. V. Efremov, S. Khim, S. Borisenko, B. Büchner, and J. van den Brink, Phys. Rev. B 93, 201101(R) (2016).

[62] I. Belopolski, P. Yu, D. S. Sanchez, Y. Ishida, T.-R. Chang, S. S. Zhang, S.-Y. Xu, H. Zheng, G. Chang, G. Bian et al., Nat. Commun. 8, 942 (2017).

[63] L. Elcoro, B. Bradlyn, Z. Wang, M. G. Vergniory, J. Cano, C. Felser, B. A. Bernevig, D. Orobengoa, G. de la Flor, and M. I. Aroyo, J. Appl. Crystallogr. 50, 1457 (2017).

[64] S. C. Miller and W. F. Love, Tables of Irreducible Representations of Space Groups and Co-representations of Magnetic Groups (Pruett, Boulder, 1967).

[65] J.-O. Willerström and S. Rundqvist, J. Solid State Chem. 39, 128 (1981). 
[66] K. Schubert, A. Raman, and W. Rossteutscher, Sci. Nat. 51, 506 (1964).

[67] S. Yatsenko, K. Tschuntonow, A. Orlow, Y. Yarmolyuk, and Y. Hryn, J. Less-Common Met. 108, 339 (1985).

[68] Z.-C. Dong and J. D. Corbett, Inorg. Chem. 35, 2301 (1996).

[69] Z.-C. Dong and J. D. Corbett, J. Cluster Sci. 6, 187 (1995).

[70] Z.-C. Dong and J. D. Corbett, Inorg. Chem. 35, 1444 (1996).

[71] Z. Wang, A. Alexandradinata, R. J. Cava, and B. A. Bernevig, Nature (London) 532, 189 (2016).

[72] G. Gatti, D. Gosálbez-Martínez, S. S. Tsirkin, M. Fanciulli, M. Puppin, S. Polishchuk, S. Moser, L. Testa, E. Martino, S. Roth, P. Bugnon, L. Moreschini, A. Bostwick, C. Jozwiak, E. Rotenberg, G. Di Santo, L. Petaccia, I. Vobornik, J. Fujii, J. Wong et al., Phys. Rev. Lett. 125, 216402 (2020).

[73] S.-M. Huang, S.-Y. Xu, I. Belopolski, C.-C. Lee, G. Chang, T.-R. Chang, B. Wang, N. Alidoust, G. Bian, M. Neupane, D. Sanchez, H. Zheng, H.-T. Jeng, A. Bansil, T. Neupert, H. Lin, and M. Z. Hasan, Proc. Natl. Acad. Sci. USA 113, 1180 (2016).

[74] L. Siggelkow, V. Hlukhyy, and T. F. Fässler, J. Solid State Chem. 191, 76 (2012).

[75] W. Wu, Z.-M. Yu, X. Zhou, Y. X. Zhao, and S. A. Yang, Phys. Rev. B 101, 205134 (2020).

[76] B. J. Wieder, Y. Kim, A. M. Rappe, and C. L. Kane, Phys. Rev. Lett. 116, 186402 (2016).

[77] B. Bradlyn, J. Cano, Z. Wang, M. G. Vergniory, C. Felser, R. J. Cava, and B. A. Bernevig, Science 353, aaf5037 (2016).

[78] T. Malakhovska, M. Y. Sabov, E. Y. Peresh, V. Pavlyuk, and B. Marciniak, Chem. Met. Alloys 2, 15 (2009).

[79] A. H. Reshak, Z. A. Alahmed, I. E. Barchij, M. Y. Sabov, K. J. Plucinski, I. V. Kityk, and A. O. Fedorchuk, RSC Adv. 5, 102173 (2015).

[80] L. Fu and C. L. Kane, Phys. Rev. B 76, 045302 (2007).

[81] T. Morimoto and A. Furusaki, Phys. Rev. B 89, 235127 (2014).
[82] Y. X. Zhao, A. P. Schnyder, and Z. D. Wang, Phys. Rev. Lett. 116, 156402 (2016).

[83] E. Janssen and G. Wiegers, J. Less-Common Met. 57, P47 (1978).

[84] Y. Xia, X. Cai, and G. Li, Phys. Rev. B 102, 041201(R) (2020).

[85] L. Wu, F. Tang, and X. Wan, Phys. Rev. B 102, 035106 (2020).

[86] S. Ponou, T. F. Fässler, G. Tobas, E. Canadell, A. Cho, and S. C. Sevov, Chem. Eur. J. 10, 3615 (2004).

[87] R. Yu, H. Weng, Z. Fang, X. Dai, and X. Hu, Phys. Rev. Lett. 115, 036807 (2015).

[88] Y. Du, F. Tang, D. Wang, L. Sheng, E.-j. Kan, C.-G. Duan, S. Y. Savrasov, and X. Wan, npj Quantum Mater. 2, 3 (2017).

[89] D. Shao, H. Wang, T. Chen, P. Lu, Q. Gu, L. Sheng, D. Xing, and J. Sun, npj Comput. Mater. 5, 53 (2019).

[90] W. Meng, Y. Liu, X. Zhang, X. Dai, and G. Liu, Phys. Chem. Chem. Phys. 22, 22399 (2020).

[91] T. F. Fässler and C. Kronseder, Angew. Chem. International Ed. 37, 1571 (1998).

[92] M. Xiao and S. Fan, arXiv:1709.02363; M. Xiao, L. Ye, C. Qiu, H. He, Z. Liu, and S. Fan, Sci. Adv. 6, eaav2360 (2020).

[93] O. Türker and S. Moroz, Phys. Rev. B 97, 075120 (2018).

[94] T. Bzdušek and M. Sigrist, Phys. Rev. B 96, 155105 (2017).

[95] M. Kim, D. Lee, D. Lee, and J. Rho, Phys. Rev. B 99, 235423 (2019).

[96] Z.-M. Yu, W. Wu, Y. X. Zhao, and S. A. Yang, Phys. Rev. B 100, 041118(R) (2019).

[97] M. A. Wilde, M. Dodenhöft, A. Niedermayr, A. Bauer, M. M. Hirschmann, K. Alpin, A. P. Schnyder, and C. Pfleiderer (unpublished).

[98] W. Wu, Y. Liu, S. Li, C. Zhong, Z.-M. Yu, X.-L. Sheng, Y. X. Zhao, and S. A. Yang, Phys. Rev. B 97, 115125 (2018).

[99] T. Fukui, Y. Hatsugai, and H. Suzuki, J. Phys. Soc. Jpn. 74, 1674 (2005). 